\title{
Systematic Optimization of Multiple Voltage Domain DC Distribution Architectures
}

\author{
by \\ Kyle Jackson Goodrick \\ B.S., University of Tennessee Knoxville, 2017 \\ M.S., University of Colorado Boulder, 2020 \\ A thesis submitted to the \\ Faculty of the Graduate School of the \\ University of Colorado in partial fulfillment \\ of the requirements for the degree of \\ Doctor of Philosophy \\ Department of Electrical, Computer and Energy Engineering
}

2021

Committee Members:

Dragan Maksimović, Chair

Robert W. Erickson

Emiliano Dall'Anese

Fabio Somenzi

Shalom Ruben 
Goodrick, Kyle Jackson (Ph.D., Electrical Engineering)

Systematic Optimization of Multiple Voltage Domain DC Distribution Architectures

Thesis directed by Prof. Dragan Maksimović

Complex electronic systems often require a power distribution architecture that provides multiple, separate voltage domains for various subsystem loads such as microprocessor cores, interface, memory, analog, and radio frequency components. The multiple point-of-load regulated voltages are typically generated using multiple dc-dc converters operating from a single input dc voltage. This thesis examines the systematic optimization of multiple voltage domain dc distribution architectures using commercially available or custom single-input single-output dc-dc converter building blocks. Three techniques are presented for this purpose: The first, an exhaustive search process called the Permutation Graph Method, enumerates possible converter arrangements and selects the converter blocks to achieve the best system figure of merit in terms of system efficiency, size, cost, power density, or a combination of these metrics. This technique is effective for systems with fewer than single digit outputs, but in larger systems becomes intractable. To solve these large problems two techniques are presented which move through the design space efficiently and find the optimal solution without exhaustive enumeration. The first is inspired by descent-based optimization and the second is based on a probabilistic optimization technique called simulated annealing. This thesis details their operation and implementation, analyzes their performance from a theoretical viewpoint, and through representative cases studies validates their performance compared to the state-of-the-art. The thesis concludes with a discussion on the use of machine learning techniques for the design and optimization of power electronics systems. 


\section{Acknowledgements}

I am grateful to my advisor, Prof. Dragan Maksimović, for his guidance and support over the last four years. I could not have asked for a better experience and I have no doubt this thesis would be as good without his excellent advice, suggestions, and numerous edits.

I would also like to thank everyone in the CoPEC lab for their assistance in all manner of things throughout the course of this work along with interesting and entertaining discussions.

Thanks also to the members of my committee, Profs. Robert Erickson, Emiliano Dall'Anese, Fabio Somenzi, and Shalom Ruben for their helpful comments, questions, and advice.

Additionally, I would like to thank Tom Byrd for the support and resources required to complete the research in this thesis.

Finally, I must thank my parents, Cecil and Karen, for their support and encouragement throughout my life. 


\section{Contents}

\section{Chapter}

1 Introduction $\quad 1$

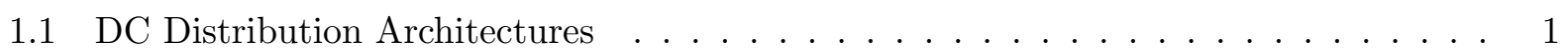

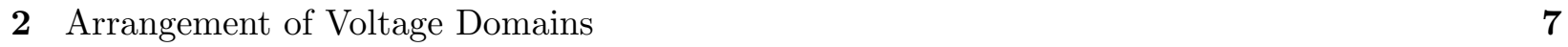

2.1 Possible Graphs . . . . . . . . . . . . . . . . . . . . . 7

2.1 .1 Number of Possible Graphs . . . . . . . . . . . . . . . . 8

2.2 Spanning Trees . . . . . . . . . . . . . . . . . . . 9

2.2 .1 Number of Spanning Trees _. . . . . . . . . . . . . . . . . . 9

2.3 Bucking and Boosting Trees . . . . . . . . . . . . . . . . . 9

2.3.1 Number of Bucking and Boosting Trees . . . . . . . . . . . . . . 10

2.3.2 Derivation of the Number of Bucking Trees . . . . . . . . . . . . 10

2.4 Design Space Heuristics _ . . . . . . . . . . . . . . . . . . . . . . 12

2.4 .1 Super Nodes . . . . . . . . . . . . . . . . . . 12

2.4 .2 Edge Removal . . . . . . . . . . . . . . . . . . . 14

2.4 .3 Intermediate Bus Nodes . . . . . . . . . . . . . . . . . . . . 15

3 Converter Selection $\quad 17$

3.1 Inclusion of Custom Converter Modules . . . . . . . . . . . . . . . . . 18

4 Architecture Ranking $\quad 19$

5 Exhaustive Search Optimization 23 
5.1 Enumeration of Bucking or Boosting Trees . . . . . . . . . . . . . . . 23

5.2 Performance Comparison . . . . . . . . . . . . . . 25

5.2 .1 All Voltages $10 \mathrm{~W} \ldots \ldots \ldots \ldots \ldots$

5.2 .2 Low Voltages $15 \mathrm{~W}-$ Others $5 \mathrm{~W} \ldots \ldots \ldots \ldots$

5.2 .3 All Voltages $25 \mathrm{~W} \ldots \ldots \ldots \ldots \ldots \ldots$

5.2 .4 Comparison with Prior Work . . . . . . . . . . . . . . . 33

6 Descent $\quad 35$

6.1 Design Space Structure . . . . . . . . . . . . . . . . . . 37

6.1 .1 Size of the Design Space . . . . . . . . . . . . . . . . . 39

6.2 Steepest Descent . . . . . . . . . . . . . . . . . . . . . 41

6.2 .1 Steepest Descent Scaling . . . . . . . . . . . . . . . . . . . 42

6.3 Stochastic Descent . . . . . . . . . . . . . . . . . . . 43

6.3.1 Stochastic Descent Scaling . . . . . . . . . . . . . . . . . 44

6.4 Case Studies . . . . . . . . . . . . . . . . . . . . . 45

6.4 Local Minima . . . . . . . . . . . . . . . . . . . 45

6.4.2 Stochastic Descent Neighbors . . . . . . . . . . . . . . . . 46

6.4.3 Performance Comparison . . . . . . . . . . . . . . . . 47

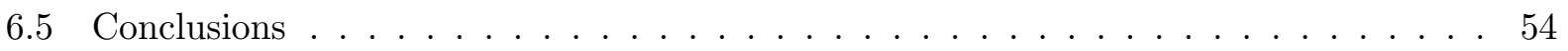

7 Simulated Annealing $\quad 55$

7.1 Simulated Annealing Review _. . . . . . . . . . . . . . . . 55

7.2 Optimization Algorithm Using Simulated Annealing . . . . . . . . . . . . . . 57

7.2 .1 Input System Structure . . . . . . . . . . . . . . . . 57

7.2 .2 Initial Configuration . . . . . . . . . . . . . . . . 58

7.2 .3 Choosing the Initial Temperature . . . . . . . . . . . . . . . . 58

7.2 .4 Perturbing the System . . . . . . . . . . . . . . . . . 59

7.2 .5 Checking Equilibrium . . . . . . . . . . . . . . . 59 


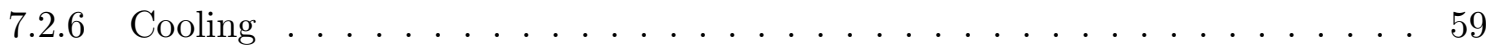

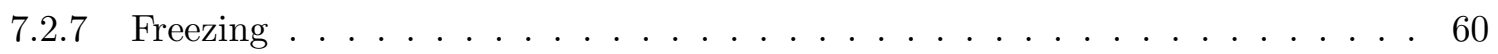

7.2 .8 System Evaluation . . . . . . . . . . . . . . . . 60

7.2 .9 Parameter Tuning . . . . . . . . . . . . . . . . . 6 61

7.3 Case Studies . . . . . . . . . . . . . . . . . . . . . 62

7.3 .1 Small Test Case . . . . . . . . . . . . . . . . . . . 64

7.3 .2 Medium Test Case . . . . . . . . . . . . . . . 66

7.3 .3 Large Test Case . . . . . . . . . . . . . . . . 66

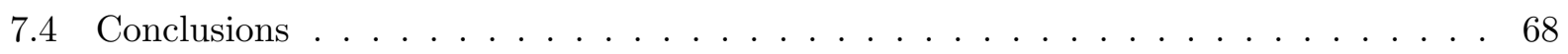

8 Case Studies $\quad \mathbf{7 0}$

8.1 Accuracy of Area Estimate . . . . . . . . . . . . . . 70

8.2 Effect of Super Nodes . . . . . . . . . . . . . . . . . . . 70

8.3 Effect of the Maximum Number of Cascaded Stages _ . . . . . . . . . . . . 71

8.4 Sensitivity to Converter Modeling Errors . . . . . . . . . . . . . . 72

8.5 Optimal Intermediate Bus Node Placement . . . . . . . . . . . . . . 73

8.6 Inclusion of Custom Converter Blocks . . . . . . . . . . . . . . . . . 74

9 Experimental Results $\quad 79$

10 Power Electronics Performance Models $\quad \mathbf{8 5}$

10.1 DC-DC Converter Performance Model . . . . . . . . . . . . . . . . . . 86

10.2 Comparison of Machine Learning Estimators for Converter Performance . . . . . . 87

10.2 .1 Classification . . . . . . . . . . . . . . . . 88

10.2 .2 Regression . . . . . . . . . . . . . . . . . . 89

10.3 Detailed Examination of the Techniques Selected for Classification and Regression . 89

10.3.1 Feature Engineering . . . . . . . . . . . . . . . 91

10.3.2 Extrapolation Performance . . . . . . . . . . . . . . 94

10.4 Application Case Studies _ . . . . . . . . . . . . . . . . . 96 
10.4.1 Intermediate Bus Node Placement . . . . . . . . . . . . . . . 96

10.4.2 DC Distribution Architecture Design . . . . . . . . . . . . . . . 96

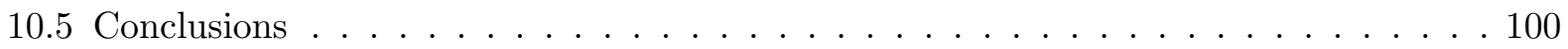

11 Conclusions $\quad 101$

11.1 Contributions . . . . . . . . . . . . . . . . . . . . 102

11.2 Future Work . . . . . . . . . . . . . . . . . . . . 102

$\begin{array}{ll}\text { Bibliography } & 104\end{array}$

\section{Appendix}

A Full Tree Generation Pseudocode 


\section{Tables}

\section{Table}

5.1 WEBENCH $[29]$ converters for all voltages at $10 \mathrm{~W} \ldots \ldots \ldots \ldots$

5.2 Permutation-Graph (PG) method converters for all voltages at $10 \mathrm{~W} \ldots \ldots$

5.3 WEBENCH $[29]$ converters for low voltages at $15 \mathrm{~W} \ldots \ldots \ldots \ldots$

$5.4 \quad \mathrm{PG}$ method converters for low voltages at $15 \mathrm{~W} \ldots \ldots \ldots \ldots$

5.5 WEBENCH $[29]$ converters for all voltages at $25 \mathrm{~W} \ldots \ldots \ldots \ldots$

$5.6 \quad \mathrm{PG}$ method converters for all voltages at $25 \mathrm{~W} \ldots \ldots \ldots \ldots$

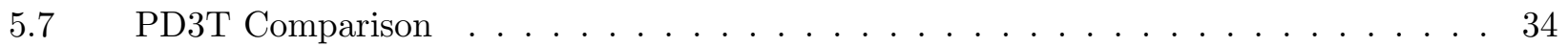

$6.1 \quad$ Probability of Meeting Performance Tolerance . . . . . . . . . . . . . . . . 45

$6.2 \quad$ Average Solve Time . . . . . . . . . . . . . . . . . . . . . 49

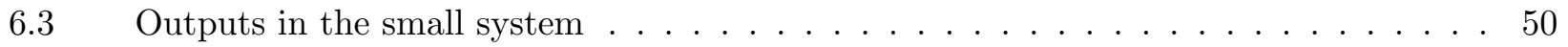

6.4 Outputs in the medium system $\ldots \ldots \ldots \ldots \ldots \ldots$

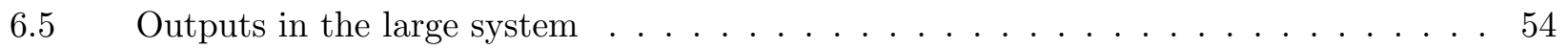

$7.1 \quad$ Simulated Annealing Hyperparameters . . . . . . . . . . . . . . . . 61

$7.2 \quad$ Average Solve Time . . . . . . . . . . . . . . . . . . . 62

$8.1 \quad$ Area Estimate Comparison . . . . . . . . . . . . . . . . . 70

$8.2 \quad$ Effect of Super Nodes on Test Cases . . . . . . . . . . . . . . . . . . . . 74

8.3 Model Based System Comparison . . . . . . . . . . . . . . . . 75

$9.1 \quad$ Experimental System Comparison . . . . . . . . . . . . . . . 80 
$9.2 \quad$ Detailed Experimental Results — COTS Only . . . . . . . . . . . . . . . 84

9.3 Detailed Experimental Results — TSAB \& COTS . . . . . . . . . . . . . 84

$9.4 \quad$ Summary Measurements . . . . . . . . . . . . . . . . . . . . . 84

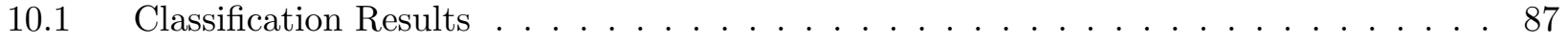

10.2 Considered Features . . . . . . . . . . . . . . . . . . 93

10.3 Regression Feature Engineering Results . . . . . . . . . . . . . . . . . . 95

10.4 Classification Feature Engineering Results . . . . . . . . . . . . . . . 95 


\section{Figures}

\section{Figure}

$1.1 \quad$ Power Electronics Applications . . . . . . . . . . . . . . . . . . . . 2

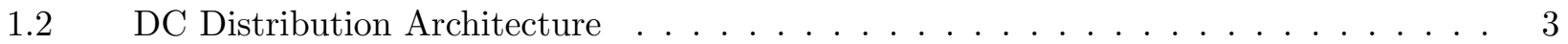

$1.3 \quad$ Example System . . . . . . . . . . . . . . . . . . . . . . 4

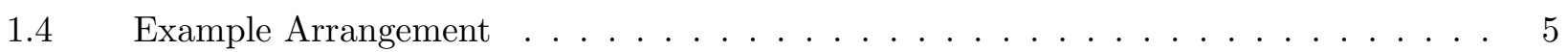

$2.1 \quad$ Number of Arrangements . . . . . . . . . . . . . . . . . . . . 8

$2.2 \quad$ Complete Graph . . . . . . . . . . . . . . . . . . . . . . 9

$2.3 \quad$ Heuristics Reduction . . . . . . . . . . . . . . . . . . . . 13

$4.1 \quad$ Example Quality Factor vs. Surface Power Density . . . . . . . . . . . . . . . . 21

$4.2 \quad$ Example Filtered Quality Factor vs. Surface Power Density . . . . . . . . . . . 21

$5.1 \quad$ Exhaustive Search Flow Chart . . . . . . . . . . . . . . . . . . . . 24

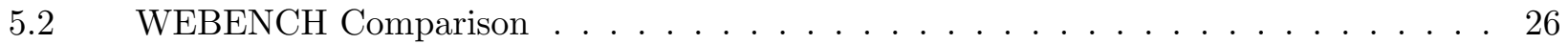

5.3 WEBENCH Comparison - Alternative Optimization Goals . . . . . . . . . . 27

$5.4 \quad$ All Voltages $10 \mathrm{~W}$ Arrangements . . . . . . . . . . . . . . . . 27

$5.5 \quad$ Low Voltages $15 \mathrm{~W}$ Arrangements . . . . . . . . . . . . . . . . . . 29

$5.6 \quad$ Converter Power Sweep . . . . . . . . . . . . . . . . . . . 30

5.7 Power Vs. Figure of Merit for two converters with different output voltages. . . . . . 30

5.8 Power Vs. Figure of Merit for a $48 \mathrm{~V}$ to $24 \mathrm{~V}$ converter. . . . . . . . . . . . . . 31

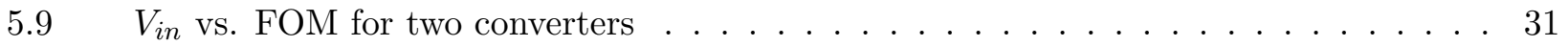

5.10 All Voltages $25 \mathrm{~W}$ Arrangements . . . . . . . . . . . . . . . . 32 
5.11 Laguna Performance Comparison . . . . . . . . . . . . . . . . . . . 34

$6.1 \quad$ Evaluated Arrangements Descent . . . . . . . . . . . . . . . . 36

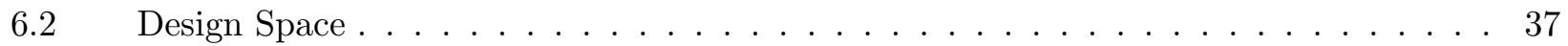

$6.3 \quad$ Descent Flow Chart . . . . . . . . . . . . . . . . . . . 38

6.4 Outcome Histogram . . . . . . . . . . . . . . . . . . 46

$6.5 \quad$ Stochastic Descent Performance . . . . . . . . . . . . . . . . . . 47

$6.6 \quad$ Descent Performance Comparison . . . . . . . . . . . . . . . . . 48

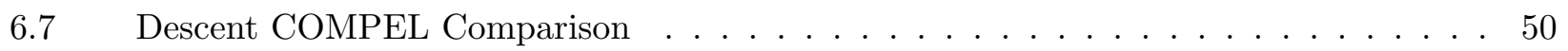

$6.8 \quad$ Small Optimal Arrangement . . . . . . . . . . . . . . . . . . . 51

6.9 Medium Optimal Arrangement . . . . . . . . . . . . . . . . . . . 51

6.10 Optimal Large Arrangement Descent . . . . . . . . . . . . . . . . 53

6.11 Descent Progress Large . . . . . . . . . . . . . . . . . . . . 53

$7.1 \quad$ Simulated Annealing Flow Chart . . . . . . . . . . . . . . . 56

$7.2 \quad$ Full Efficient Technique Comparison . . . . . . . . . . . . . . 63

7.3 Simulated Annealing COMPEL Comparison . . . . . . . . . . . . . . . 64

$7.4 \quad$ Simulated Annealing Progress — Small . . . . . . . . . . . . . . . . . 65

7.5 Simulated Annealing Progress - Medium . . . . . . . . . . . . . 66

$7.6 \quad$ Large Optimal Arrangement . . . . . . . . . . . . . . . . . 68

$7.7 \quad$ Simulated Annealing Progress — Large . . . . . . . . . . . . . . . . . . 69

$8.1 \quad$ Area Comparison Layout . . . . . . . . . . . . . . . . . 71

$8.2 \quad$ Depth Comparison . . . . . . . . . . . . . . . . . 72

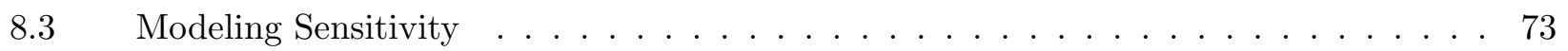

$8.4 \quad$ Number of Intermediate Bus Nodes . . . . . . . . . . . . . . . . . . . . 74

8.5 TSAB Test Case Block Diagram . . . . . . . . . . . . . . . . 75

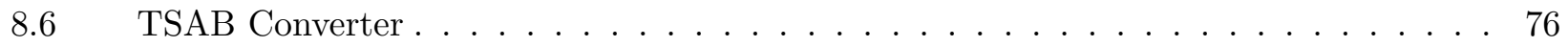

$8.7 \quad$ Experimental System Block Diagrams . . . . . . . . . . . . . . . . 77 
8.8 TSAB Power Density vs. Quality factor Pareto Fronts . . . . . . . . . . . 78

$9.1 \quad$ Hardware Prototypes . . . . . . . . . . . . . . . . . . . . . . 80

$9.2 \quad$ Experimental Test Setup . . . . . . . . . . . . . . . . . . 80

$9.3 \quad$ Load Board . . . . . . . . . . . . . . . . . . . . . 81

$9.4 \quad$ Load Board Bottom . . . . . . . . . . . . . . . . . . . 82

$9.5 \quad$ Control Board . . . . . . . . . . . . . . . . . . . . . 82

$9.6 \quad$ Experimental Setup Block Diagram . . . . . . . . . . . . . . . . . . 83

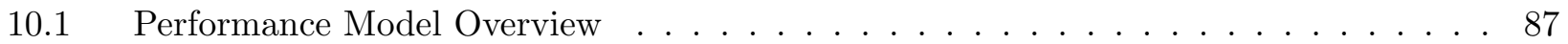

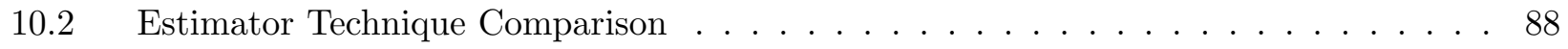

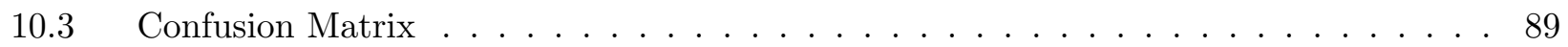

10.4 Estimator Test Performance . . . . . . . . . . . . . . . . . . . . . 90

$10.5 \quad$ Estimator Sweep Performance . . . . . . . . . . . . . . . . . . . . . 92

$10.6 \quad$ Estimator Correlation Matrix _. . . . . . . . . . . . . . . 94

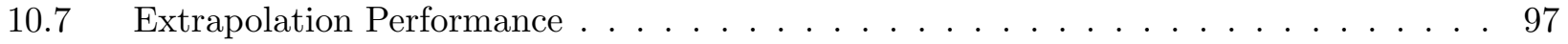

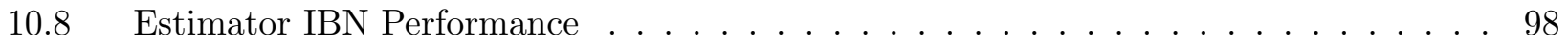

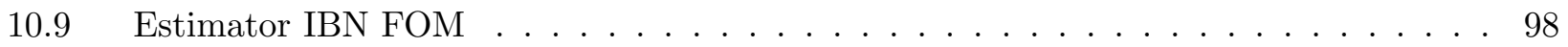

10.10 Estimator System Performance _. . . . . . . . . . . . . . . . . . . 99 


\section{List of Algorithms}

\section{Algorithm}

5.1 Simplified Bucking Trees . . . . . . . . . . . . . . . 25

6.1 Size of the Design Space . . . . . . . . . . . . . . . . . . . . . 42

A.1 Complete algorithm for finding all possible bucking and boosting trees . . . . . . . 119 


\section{Chapter 1}

\section{Introduction}

Power Electronics is an enabling technology for the $21^{\text {st }}$ century. By 2030 it is estimated that up to $80 \%$ of electrical power will flow through some form of power electronics before its final use [1]. The applications of power electronics are broad and a selection of examples is shown in Fig. 1.1. In many of these applications there are often more than one load that requires power and these various loads often require different voltages. However, the required voltages are frequently not readily available and must be generated from a single available power source through some power electronics architecture. Motivated by the need to design these systems this thesis describes the systematic design and optimization of single input multiple output dc distribution architectures.

\subsection{Distribution Architectures}

Many electronic systems, including server, telecom, embedded, mobile, aerospace, and automotive platforms, require multiple tightly regulated point-of-load dc power supply voltages for diverse system components such as microprocessor cores, memory, interface, analog, display, and radio-frequency subsystems. A block diagram of such a dc distribution architecture is shown in Fig. 1.2, and a practical example where five regulated point-of-load dc voltages: $\{1,3.3,5,12$, and 24\} $\mathrm{V}$ are generated from a $48 \mathrm{~V}$ input dc voltage is shown in Fig. 1.3, including typical loads.

Various custom single-input multiple-output (SIMO) dc-dc converters present approaches to realization of multiple-output DC distribution architectures, which may offer advantages in certain applications [2]-[9]. In most cases, however, multiple-output DC distribution architectures are based on arrangements of multiple single-input, single-output (SISO) converters, where SISO 


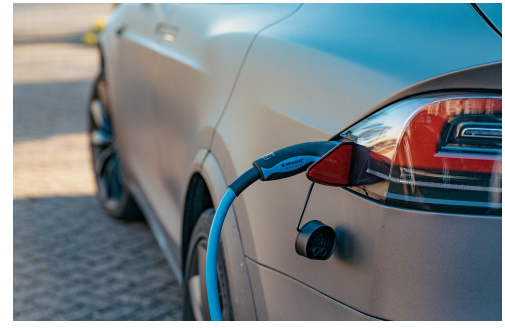

(a) Electric Vehicles

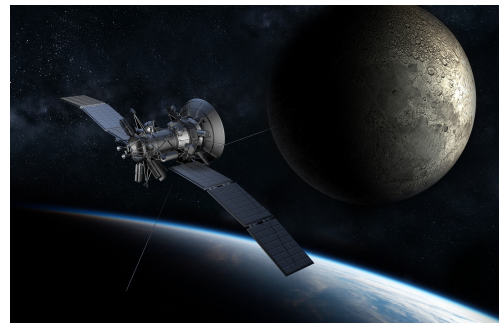

(d) Aerospace

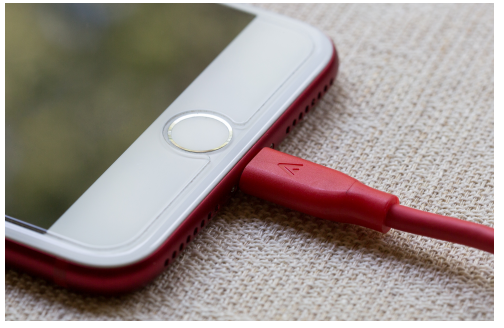

(b) Mobile

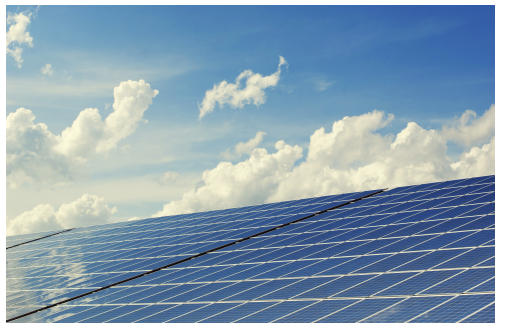

(e) Solar Power

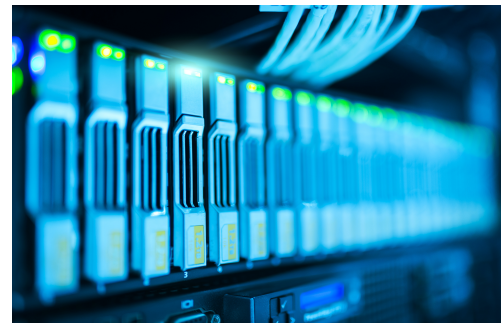

(c) Data Centers

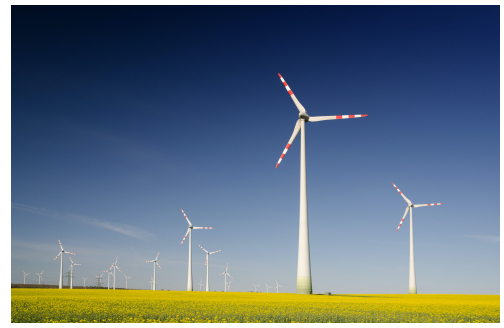

(f) Wind Power

Figure 1.1: Example applications of power electronics. (a) Power electronics is required in all aspects of electric vehicles: charging, motor drive, battery management, and accessory power. (b) Power electronics is used externally to charge mobile devices and also internally to process power for the various subsystems. (c) Power electronics is used extensively in data centers for distribution and in point of Load regulators powering the various server components. (d) Aerospace applications make use of power electronics for processing of photovoltaic power and to power the various required subsystems. (e) Power electronics is used to maximize the power produced by PV cells and also to connect it to the grid. (f) Power electronics is required to process wind power and connect it to the grid.

converter blocks can be standard or custom single stage, paralleled, interleaved or composite converters, as illustrated in Fig. 1.3. In this example, the dc distribution architecture is realized using five dc-dc modules arranged so that some dc-dc modules are supplying both loads and downstream conversion blocks, while some dc-dc modules are serving as point-of-load regulators exclusively. It is clear that the arrangement of dc-dc converters shown in Fig. 1.3 is not unique. This thesis is focused on finding the arrangement and selection of dc-dc converters that achieve the best system Figure of Merit (FOM) in terms of system efficiency, size, cost, power density, or a combination of these metrics [10].

In the past, much attention has been given to analysis, modeling, and design optimization of individual dc-dc converter modules [11]-[23], while less attention has been given to the optimiza- 


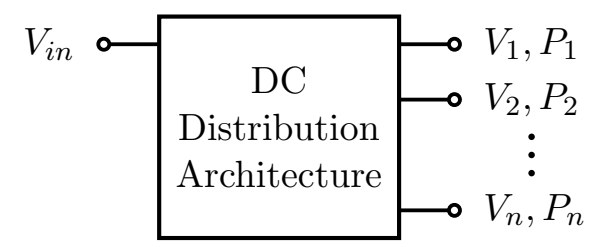

Figure 1.2: DC distribution architecture where multiple regulated point-of-load dc voltages are obtained from a single dc input source. Such architectures are required in many systems including mobile, computing, telecom, aerospace, automotive, and other applications. The architecture is typically realized using multiple single-input single-output dc-dc converter modules.

tion of dc power distribution architectures. In the well-known two-stage approach known as the Intermediate Bus Architecture [24], [25], a single intermediate bus voltage supplies all downstream point-of-load converters. However, this is not necessarily the optimal solution, and there is room for improvement by considering more complex system arrangements. A heuristic approach for designing power architectures using voltage regulator building blocks is discussed in [26], [27]. An algorithm is proposed to predict which architectures are most likely to be optimal. The solution space includes a separate solution for identical arrangements with different converter sets, as well as multiple solutions for separate loads fed from the same voltage domain. This significantly increases the number of possible solutions and increases required computational effort. The method in [26] also separates the process of generating arrangements and selecting converters. This means that any improvements in converter building blocks do not translate directly into the selection of the most desirable arrangements.

Some vendors have offered tools intended to simplify power distribution architecture design and choice of dc-dc converter realizations. One such tool was Texas Instruments (TI) WEBENCH Power Architect ${ }^{1}$ [29]. However, this tool was unable to consider arrangements with a depth of more than two cascaded converters, and was locked to converter implementations offered by the vendor.

This thesis describes a systematic method for determining the optimal architecture in nonisolated single-input multiple-output dc power distribution systems using SISO dc-dc converter

\footnotetext{
${ }^{1}$ In the time since the work reported in this thesis was completed, TI WEBENCH Power Architect has been discontinued. Analog Devices offers a tool, LTpowerPlanner [28]; however, this tool has only analysis capabilities with no system optimization functionality.
} 


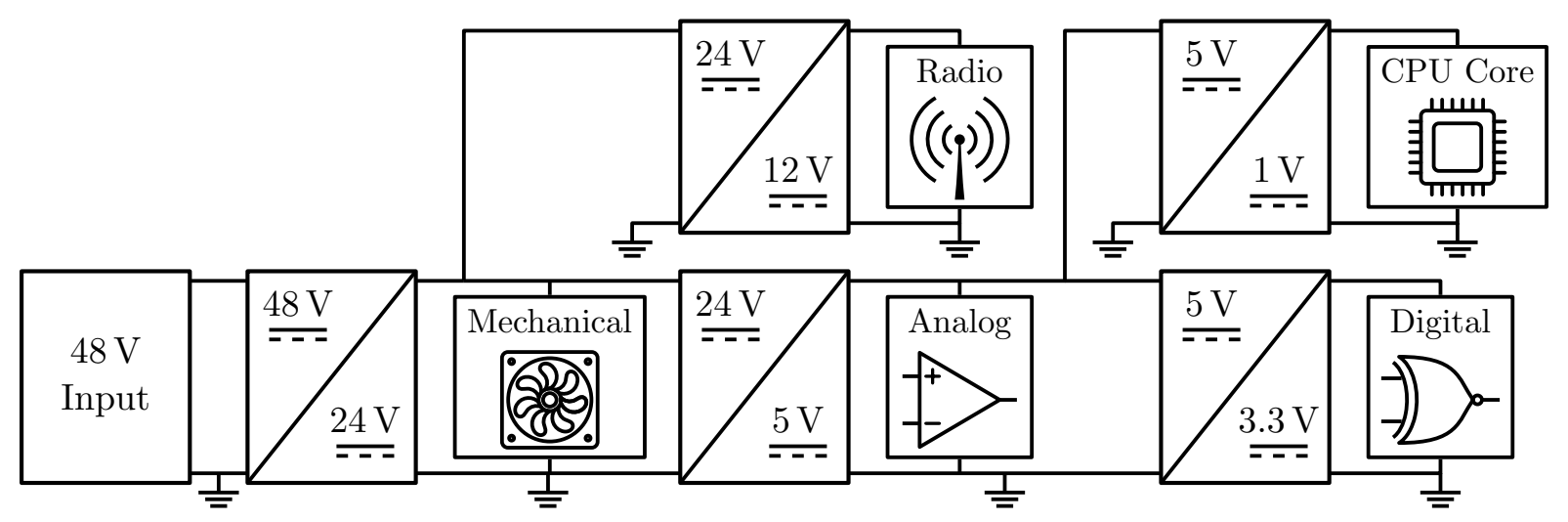

Figure 1.3: An example DC distribution architecture where multiple regulated dc voltages, $\{1$, $3.3,5,12$, and 24$\} \mathrm{V}$, are obtained from a single $48 \mathrm{~V}$ dc input source. The system example is representative of dc distribution systems in computing, telecom and other applications. The architecture is realized using an arrangement of dc-dc converter modules. Typical loads at each voltage domain are also shown. This system's arrangement in graph form is shown in Fig. 1.4a.

modules. A new approach, the PG method [10], is proposed to generate the possible arrangements and select converters without enumerating all possible combinations of converter sets. This reduces computational effort and allows determination of the optimal architecture without reliance on heuristics, and without restrictions on the depth of cascaded converters. Representative test cases are used to demonstrate substantial improvements compared to the solutions offered by [27], [29]. As an example, the approach is applied to a system that requires five regulated point-of-load voltages $\{1,3.3,5,12,24\} \mathrm{V}$ from a $48 \mathrm{~V}$ input dc voltage. Two experimental system prototypes are constructed to illustrate the optimization results: (i) a prototype using only Commercial Off The Shelf (COTS) converter blocks and (ii) a prototype with custom designed converter blocks along with COTS blocks. It is shown how the proposed optimization method finds an alternate arrangement that takes advantage of the custom converter characteristics to achieve a $179 \%$ improvement in a system Figure of Merit compared to the COTS-only based system, where the considered FOM is inversely proportional to system losses and footprint area. Additional analysis shows that the FOM improvement is mainly due to the different arrangement, not just due to the improved custom converters.

Additionally, this thesis presents two classes of efficient optimization techniques that may be used to optimize systems that are too large to be optimized using an exhaustive search. The 


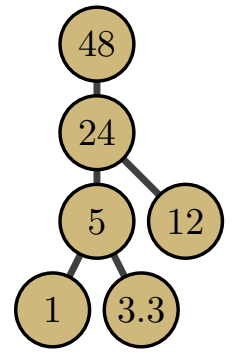

(a)

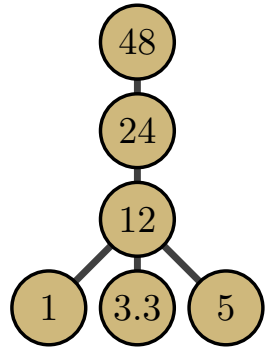

(b)

Figure 1.4: Graph representations of two possible arrangements for a system where the input voltage is $48 \mathrm{~V}$ and the output voltages are $24,12,5,1$, and $3.3 \mathrm{~V}$ : (a) the graph for the arrangement shown in a block-diagram form in Fig. 1.3, and (b) the graph for an alternative arrangement. The nodes in the graph represent separate voltage domains while the edges represent dc-dc converters.

first class uses descent based optimization to design systems based on a design space that allows the optimal system to be found without evaluating all possible arrangements. The second class, based on optimization by simulated annealing, uses a probabilistic optimization approach to move through the design space even faster than using descent based methods.

Finally, the thesis discusses machine learning techniques that may be applied to power electronics system design problems and describes their performance.

This thesis is organized in eleven chapters:

- Chapter 1 introduces the problem and motivates the work

- Chapter 2 describes how dc distribution architectures are arranged and provides theoretical analysis of these arrangements

- Chapter 3 details how arrangements are turned into architectures by selecting converters

- Chapter 4 discusses how different architectures are compared to one another and gives example results

- Chapter 5 describes an exhaustive search approach to optimizing de distribution architectures 
- Chapter 6 presents an efficient descent based optimization approach with significant computational advantages

- Chapter 7 details an additional efficient optimization technique based on simulated annealing with further performance benefits

- Chapter 8 presents a series of case studies that showcase the benefits of systematic dc distribution optimization

- Chapter 9 presents experimental results for a case study

- Chapter 10 describes the use of estimators in the design and optimization of dc distribution architectures

- Chapter 11 Concludes the thesis 


\section{Chapter 2}

\section{Arrangement of Voltage Domains}

In DC distribution architectures the arrangement is the structural component of the architecture describing the connections present in the system. To generate the required regulated dc outputs, single-input single-output dc-dc converters can be arranged in many different ways. The architecture could be as simple as having each output generated by a converter supplied from the system input voltage, or the converters could form a more complicated multilayer tree. While multilayer trees reduce the conversion ratio required for many of the converters there is a tradeoff as the power supplied to cascaded converters must be processed more than once. The possible arrangements can be represented as graphs with nodes corresponding to the input and required voltage domains and edges representing the converters, as illustrated in Fig. 1.4 where two possible arrangements are shown for a system that has the same voltage requirements as the system example in Fig. 1.3.

Arrangements can be enumerated in several ways and three are described in Sections 2.1 to 2.3. Additionally, heuristics can modify the design space as discussed in Section 2.4.

\subsection{Possible Graphs}

A possible graph is one arrangement of converters for a given set of required outputs. A list of possible graphs can be created by constructing a complete graph of the voltage domains present in the system and finding all subgraphs of the complete graph that contain $n_{o}$ edges, where $n_{o}$ is the number of output voltages in the system. The complete graph of a system with six outputs is shown in Fig. 2.2 using solid, gold edges. In this example the possible graphs would be all combinations 


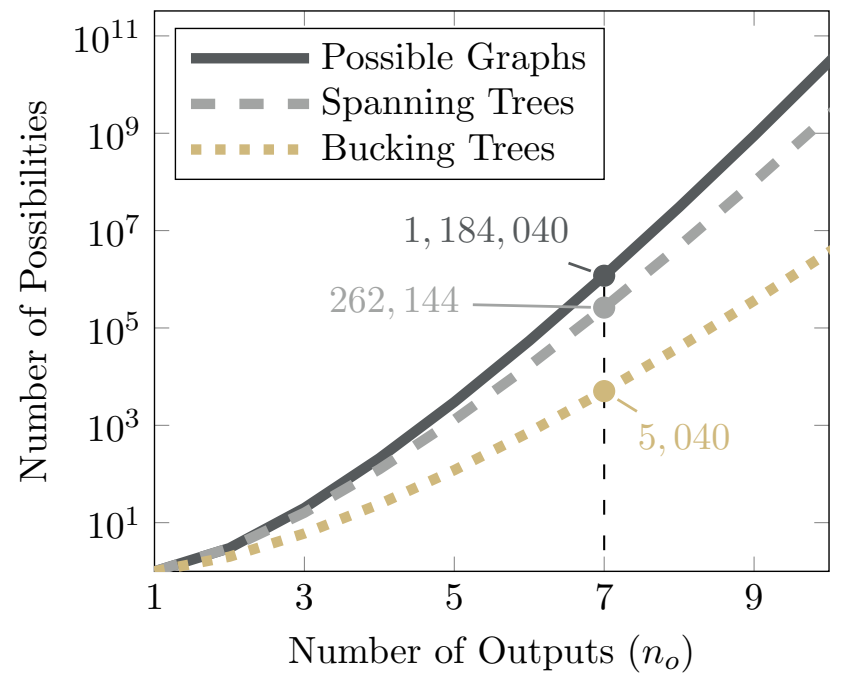

Figure 2.1: Number of possible graphs, spanning trees, and bucking trees as functions of the number of outputs $n_{o}$. Marks indicate the total number of possibilities of each type for $n_{o}=7$.

of six gold edges.

\subsubsection{Number of Possible Graphs}

The number of possible graphs can be found as the number of combinations of $n_{o}$ edges chosen from the $n_{e}$ edges of the complete graph. This may be calculated with

$$
\text { Number of Possible Graphs }=\left(\begin{array}{l}
n_{e} \\
n_{o}
\end{array}\right)=\frac{n_{e} !}{n_{o} !\left(n_{e}-n_{o}\right) !}
$$

where the number of edges in the complete graph, $n_{e}$, can be calculated using

$$
n_{e}=\frac{n(n-1)}{2}
$$

where $n=n_{o}+1$ is the total number of nodes in the complete graph.

Fig. 2.1 shows the rapid increase in the number of possibilities as the number of outputs increases. The list of possible graphs contains all possible arrangements of the output voltages; however, not all arrangements are desirable or physically realizable. To reduce the computational effort, there is a strong incentive to construct a subset of the list of all possible graphs, which leads to the concept of "Spanning Trees." 


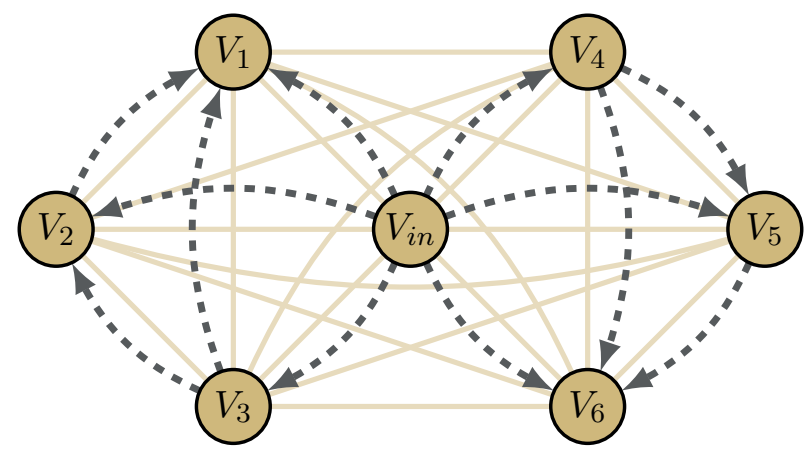

Figure 2.2: Complete graph for a system with six outputs, $V_{1}<V_{2}<V_{3}<V_{\text {in }}<V_{4}<V_{5}<V_{6}$. The solid gold lines connect every node to every other node to form the graph used with possible graphs and spanning trees, while the dashed grey lines represent the characteristic graphs used with bucking and boosting trees. The bucking and boosting characteristic graphs show how the problem can be separated into two decoupled, smaller problems.

\section{$2.2 \quad$ Spanning Trees}

Spanning trees is a subset of the full list of possible graphs. Each spanning tree is taken from the complete graph of the input and outputs, as illustrated in the graph shown in Fig. 2.2 using the solid, gold edges. A spanning tree contains all the voltages, each with a path to the input, and does not contain any loops [30].

\subsubsection{Number of Spanning Trees}

The number of spanning trees can be found using Cayley's formula [31],

$$
\text { Number of Spanning Trees }=\left(n_{o}+1\right)^{n_{o}-1} \text {. }
$$

The number of spanning trees is compared to the number of possible graphs in Fig. 2.1. One may note that the number of spanning trees can still be very large.

\subsection{Bucking and Boosting Trees}

In most cases of interest, the point-of-load voltages required are always lower or always higher than the available input voltage. Specifically, consider the case when all voltages are less than the input voltage. In such cases it would likely be inefficient to cascade step-up and step-down converter 
stages. A similar assumption may be made for voltages greater than the input. This leads to the concept of "Bucking and Boosting Trees," which are spanning trees that contain only monotonic cascaded converter stages, i.e., no stages that boost up after bucking down or vice versa. This effectively splits the optimization problem into two smaller problems. As an example, in Fig. 2.2 the bucking and boosting trees are constructed by finding the spanning arborescences of the directed graph with the grey, dashed edges.

\subsubsection{Number of Bucking and Boosting Trees}

The number of bucking or boosting trees can be found simply as the number of permutations of the output voltage domains,

$$
n_{\text {trees }}=n_{o} !,
$$

which yields a substantial reduction in the number of possibilities to be considered, as illustrated in Fig. 2.1. It should be noted that, for fair comparison, the numbers in Fig. 2.1 are found assuming that all output are either less than or greater than the input voltage.

\subsubsection{Derivation of the Number of Bucking Trees}

The result (2.4) can be derived by representing the system as a directed graph and applying the Tutte's Matrix-Tree Theorem [32], [33]. To represent the system as a directed graph, consider a characteristic graph (similar to that shown with grey, dashed edges in Fig. 2.2) that contains all of the possible connections within a system with all output voltage domains lower than the input. The graph has a node for the input voltage and one for each output. Each node has edges that go from it to each voltage domain lower than it (i.e. all nodes that could be reached by bucking.) In order to apply the Tutte's Matrix-Tree Theorem, this characteristic graph must be described in matrix form using adjacency matrix $M$. Each row of $M$ represent a node in the graph. For the systems described in this thesis, without loss of generality, the input is represented by the first row followed by the outputs in descending order. Each row in the adjacency matrix describes the connections from that node to the other nodes in the characteristic graph; a 1 indicates that an edge exists and a 
0 indicates that one does not. In order to apply the Matrix-Tree Theorem, a degree in matrix, $D_{i n}$, is constructed, which is a diagonal matrix where each element in the principal diagonal represents the number of edges going into that row's node. This can be determined by summing each column of the adjacency matrix. For the characteristic graph the matrices $M$ and $D_{i n}$ have the following form:

$$
\begin{aligned}
M & =\left[\begin{array}{lllll}
0 & 1 & 1 & \cdots & 1 \\
0 & 0 & 1 & \cdots & 1 \\
0 & 0 & 0 & \cdots & 1 \\
\vdots & \vdots & \vdots & \ddots & \vdots \\
0 & 0 & 0 & \cdots & 0
\end{array}\right] \\
D_{i n} & =\left[\begin{array}{lllll}
0 & 0 & 0 & \cdots & 0 \\
0 & 1 & 0 & \cdots & 0 \\
0 & 0 & 2 & \cdots & 0 \\
\vdots & \vdots & \vdots & \ddots & \vdots \\
0 & 0 & 0 & \cdots & n_{o}
\end{array}\right]
\end{aligned}
$$

Each bucking tree is, in graph theory terms, a spanning arborescence of the characteristic graph, i.e. a graph rooted at a particular node (the input) with directed edges connecting the input node to each node in the characteristic graph. Application of the Tutte's Matrix-Tree Theorem counts the number of spanning arborescences in the characteristic graph that are rooted at the input node. From this theorem the number of possible arborescences rooted at the $j^{t h}$ node is

$$
N_{j}=\operatorname{det}\left(\hat{L}_{j}\right)
$$

where

$$
L=D_{i n}-M
$$

and $\hat{L}_{j}$ is $L$ with the $j^{\text {th }}$ row and column removed.

Substituting (2.5) and (2.6) into (2.8), and removing the first row and column (because the 
input is the root of all the bucking tree arborescences) results in:

$$
\hat{L}_{j}=\left[\begin{array}{cccc}
1 & -1 & \cdots & -1 \\
0 & 2 & \cdots & -1 \\
\vdots & \vdots & \ddots & \vdots \\
0 & 0 & \cdots & n_{o}
\end{array}\right]
$$

Finally, the number of bucking trees may be calculated by substituting (2.9) into (2.7). Since

$\hat{L}_{j}$ is a triangular matrix, the determinant is easily calculated as the product of the elements on the principal diagonal. As each element on the diagonal is the row number, the determinant equals the number of rows factorial or $n_{o}$ !, as shown in (2.4).

It is simple to reverse the logic to get the same result for boosting trees. The total number of trees can then be found by adding the number of bucking trees to the number of boosting trees.

\section{$2.4 \quad$ Design Space Heuristics}

In certain cases there are systems where the number of required voltage domains, and therefore the size of the design space, is either too large or too small. When a large number of independently regulated outputs are required, the number of possible arrangements increases rapidly as shown in Section 2.3. In such cases, it is desirable to reduce the number of effective nodes. Conversely, when the number of required output voltages is low there are very few choices and it may be beneficial to increase the size of the design space, by adding additional, optional, voltage domains to the design space. Sections 2.4.1 and 2.4.2 describe heuristics for decreasing the size of the design space and Section 2.4.3 describes a heuristic that can increase the size.

\subsubsection{Super Nodes}

One method, leading to the concept of "super nodes," is based on the assumption that voltage domains close to each other in value will all be connected to the same parent voltage domain. By grouping close-in-value voltage domains into super nodes, the number of effective arrangements can be reduced and further optimizations can be made during the converter selection process as 


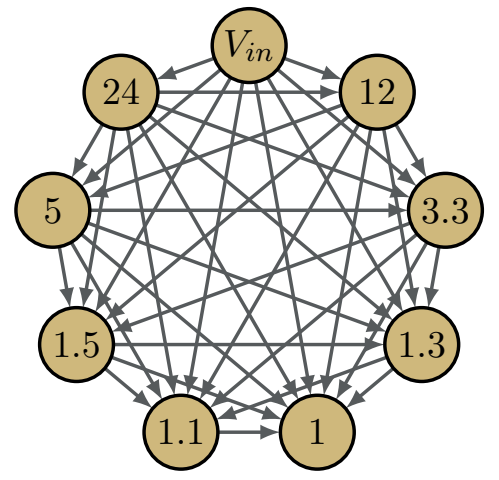

(a) Characteristic

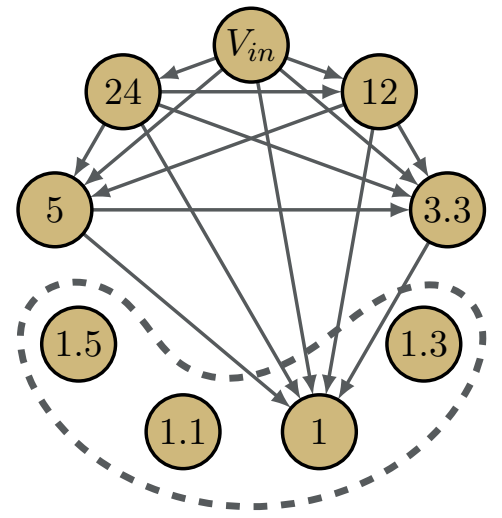

(b) Super Nodes

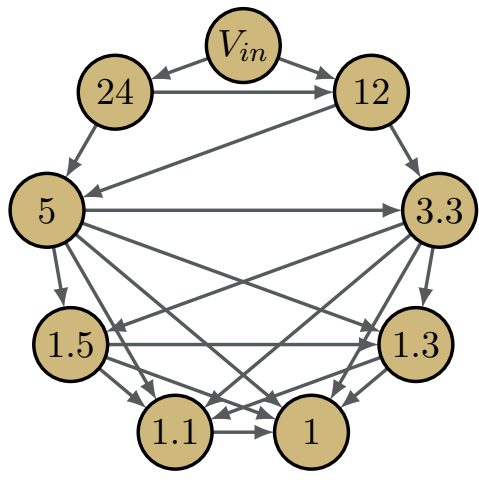

(c) Edge Removal

Figure 2.3: Example system with a large number of output nodes. (a) Shows all the possible connections when considering only bucking trees. In total there are $n_{\text {trees }}=40320$ spanning arborescences. (b) Shows an example reduction in the number of possible trees by creating a super node that includes the $1,1.1,1.3$, and $1.5 \mathrm{~V}$ nodes. In this case there are $n_{\text {trees,super }}=120$ spanning arborescences and for each one the 1.1, 1.3, and $1.5 \mathrm{~V}$ nodes will be connected to the same parent node as the $1 \mathrm{~V}$ node. (c) Shows another possible subset using the edge removal technique. In this case all edges that correspond to a conversion ratio $V_{\text {in }} / V_{\text {out }}$ greater than six are removed, which yields $n_{\text {trees }, \text { reduced }}=960$ spanning arborescences.

discussed in Chapter 3.

As an example, consider the system shown in Fig. 1.3. With five outputs there are 120 possible bucking trees. However, if new processors are added to the system that require 1.1, 1.3, and $1.5 \mathrm{~V}$ as shown in Fig. 2.3a the number of possibilities will increase to 40320, which will take considerably longer to evaluate. Noting that the new processors' voltage requirements are comparable to the previous processor's requirement a super node can be formed around the $1 \mathrm{~V}$ node as shown in Fig. 2.3b. In this case the number of possible trees will remain at 120 and each new processor's node will be attached to the same parent as the $1 \mathrm{~V}$ node.

From the analysis in Section 2.3.1, the reduction in possibilities offered by super nodes can be calculated easily. If $n_{s u b}$ is the number of nodes that are paired with a super node, the number of possible bucking trees is

$$
n_{\text {trees,super }}=\left(n_{o}-n_{\text {sub }}\right) ! .
$$

This is because the number of effective outputs is reduced by $n_{s u b}$ and therefore the last $n_{\text {sub }}$ terms 
must be removed from the expanded factorial.

The effect of combining voltage domains into super nodes is discussed in Section 8.2 for a set of test cases, illustrating a tradeoff between size of the optimization problem and the outcome in terms of the system performance.

\subsubsection{Edge Removal}

Another technique that can be used to reduce the number of possible combinations can be seen from the derivation of the number of bucking trees discussed in Section 2.3.1. If $n_{r}$ edges into a node are removed from the characteristic graph the number of possibilities is reduced to

$$
n_{\text {trees,reduced }}=\frac{P-n_{r}}{P} n_{\text {trees }}
$$

where $n_{\text {trees }}$ is the number of spanning arborescences before the edge is removed and $P$ is the index of the node in the descending sorted list of outputs. For the highest domain's node $P=1$ and $P=n_{o}$ for the the lowest node (in the bucking case). The result (2.11) can be found from (2.6) and (2.9). If $n_{r}$ edges into a node are removed, the node's entry in the $D_{i n}$ matrix will be

reduced by $n_{r}$, which will reduce its term in the determinant of $\hat{L}_{j}$ by $n_{r}$. Since the node's term in the $D_{\text {in }}$ matrix in the complete case is $P$, it follows that the number of possibilities is reduced by $\left(P-n_{r}\right) / P$, as shown in $(2.11)$.

Using (2.7), which shows that the number of spanning arborescences is the product of the principal diagonal of the $D_{i n}$ matrix, i.e. the product of the number of edges into each node, the result (2.11) can be extended to the case when edges are removed to more than one node.

Consider again the system example shown in Fig. 2.3a. Recall that an edge in the graph represents a dc-dc converter. In general, achieving high efficiency or high power density is more challenging for converters with high step-down (or high step-up) ratio. Consequently, one may consider removing all edges that correspond to conversion ratios greater than six as shown in Fig. 2.3c. This results in only 960 possible bucking trees, a reduction of $97.6 \%$ compared to the complete case in Fig. 2.3a. 
While edge removal is an effective technique to reduce the number of possible arrangements, the decision about which edges to remove is heuristic, and may not yield a solution close to the optimum. For example, if high-efficiency transformer-isolated high step-down stages (e.g. [34]-[38]) are included in the converter database, removal of the edges based on conversion ratio alone would not necessarily be well justified.

\subsubsection{Intermediate Bus Nodes}

In systems where a large step-down conversion may be required between the input and groups of outputs, one may consider adding intermediate bus nodes that are not connected directly to a load, but can lead to reduced required conversion ratios and improved system efficiency [24], [25]. The approach of including intermediate bus nodes into consideration is inspired by the fact that multi-stage solutions may outperform single-stage conversion in practice. The number of trees to be considered increases as

$$
n_{\text {trees }, \text { bus }}=\left(1+N_{p}\right) n_{\text {trees }},
$$

where $N_{p}$ is the number of nodes after the intermediate bus node in the descending ordered list containing all nodes (for the bucking trees case), including intermediate bus nodes and $n_{\text {trees }}$ is the number of possible trees before the intermediate bus node is added. It is important to note that intermediate bus nodes must feed a converter whereas output nodes may feed only their load. Therefore, trees that contain intermediate bus nodes at the end of a branch should not be considered. This can be shown by examining a system containing $n_{o}$ outputs, for which there are $n_{\text {trees }}=n_{o}$ ! bucking trees as shown in (2.4). Now consider adding an intermediate bus node whose value places it between the $2^{\text {nd }}$ to lowest and the lowest output node. As the intermediate bus node is optional, all $n_{o}$ ! trees that do not contain it are valid. Additionally, there are another $n_{o}$ ! bucking trees obtained by inserting the intermediate bus node between the lowest domain node and the node it would have been connected to without the intermediate bus voltage. To continue the pattern for a general placement of the intermediate bus, recall that each of the trees may be represented as a permutation of the list of outputs. From Algorithm 5.1 it follows that if a voltage in the 
permutation is smaller than the next voltage in the permutation, nothing will be attached to it and the next voltage will be connected to one of its parents. Therefore, to ensure that the intermediate bus node has at least one child it may only be inserted before elements in the permutation that are less than it (in the bucking case). There are $N_{p}$ places in each of the original permutations where this is the case so the total number of trees will increase by a factor of $1+N_{p}$. 


\section{Chapter 3}

\section{Converter Selection}

Once all possible bucking or boosting trees have been found, the individual converters that make up each tree must be selected. In this work, the Texas Instruments WEBENCH tool [29], along with data for custom converters based on the approach presented in [39], [40], are used to find converters for a given input and output voltage and output power.

One may note that WEBENCH tool does not include converter volume or weight data. These parameters could be added to a converter database to accommodate optimization goals in terms of volumetric power density. Furthermore, the optimization tool implementing the PG method is capable of using converter data from other sources or custom converter entries that contain information about the footprint area, efficiency curves, volume, weight, cost, or other parameters of interest. If so desired, the converter data source may be limited to existing converter designs to remove any requirement for additional work at the supply chain or regulatory level. Converter selection may also take into account efficiency curves by considering system level load profiles of interest and application dependent weights.

It is important to note that converter blocks could be any single-input, single-output dc-dc configurations, including standard single stage converters, paralleled, interleaved or composite converters. Optimization of individual converter stages has been discussed widely in the literature [11][23], it is considered a given and not explored in depth in this thesis.

For each arrangement generated as described in Chapter 2, the algorithm traverses the tree until it is at the tip of each branch. It then finds a converter for the tip and works back up the branch using the results of child converters when selecting the parent converters, thus ensuring 
that parent converters can support both the loads and losses of their children. The converter that best meets the optimization goal is chosen when selecting the converter for each edge. Only the converters that meet the specific input and output voltage and current requirements of each edge are considered during this step. Once a converter is found for all edges of each tree, it is possible to evaluate the arrangement as a whole to rank the considered architectures and determine the optimal solution.

\subsection{Inclusion of Custom Converter Modules}

Once the required input/output characteristics of the converter are determined, the optimization procedure can choose a standard configuration from a database of commercially available solutions (such as WEBENCH), or a custom converter designed for the specific conversion specifications. This custom converter module can then be compared and selected should it's performance be superior to the standard options.

In order to include custom converter options alongside the standard solutions, properties of interest for each considered topology must be determined within the PG method. In this thesis, the characteristics of interest include surface power density and efficiency; however, in other applications the properties could include area, volume, cost, etc. To characterize a custom converter, it is necessary to develop a model or experimental results that can accurately predict the losses and size of each of the power components for given input/output voltages and power level. Once the converter design procedure has determined the size of the power components, they are combined with a list of baseline components that do not vary with power or input/output voltage. The overall area can then be determined using the same method as with standard commercially available converter blocks. 


\section{Chapter 4}

\section{Architecture Ranking}

There are many ways to rank a particular architecture once the arrangement is defined and converters are selected. The simplest ways can look at only one metric, e.g., efficiency, area, power density, or cost. When using these methods, it is relatively easy to calculate the overall architecture metric. For area and cost the value can be estimated by adding up the individual cost or area of each converter. The system efficiency evaluation is slightly more complicated, but still relatively simple:

$$
\eta_{\text {tree }}=\frac{\sum_{i=1}^{o} P_{\text {out }, i}}{\sum_{i=1}^{o} P_{\text {out }, i}+P_{\text {loss }, i}}
$$

While determining the footprint area for the overall architecture given the individual converter areas is relatively straightforward, determining the area for the individual converters is more difficult. This is especially true when multiple data sources are used to determine the optimal converters. To compare converters fairly, a standard method of estimating the area must be used. In the WEBENCH tool [29], the area for each converter is found as the area of each component in the bill of materials with $1 \mathrm{~mm}$ of extra space on each side. While this method is not an accurate representation of the actual final area, it provides for a reasonably fair way to compare two different converters when determining the optimal choice. The accuracy of this area estimate is discussed further in Section 8.1.

In addition to efficiency, power density, area, size, weight, or cost, architectures can also be ranked based on a combination of two or more of the metrics in an overall FOM. The choice of 
optimization goal is dependent on the application requirements. For example, the FOM used in this thesis is defined as

$$
\mathrm{FOM}=\frac{Q}{A}=\frac{Q \rho}{P_{\text {out }}}=\frac{\rho}{P_{\text {loss }}}
$$

where

$$
Q=\frac{\eta}{1-\eta}=\frac{P_{\text {out }}}{P_{\text {loss }}}
$$

is referred to as the system quality factor, which depends on the system efficiency $\eta$,

$$
\rho=\frac{P_{\text {out }}}{A}
$$

is the surface power density, and $A$ is the footprint area. All terms refer to the overall system architecture.

Fig. 4.1 presents an example plot of all 5040 possible architecture's quality factor $Q$ vs. surface power density $\rho$ for a considered test case with 7 output voltages, and converters taken from the TI WEBENCH database. In this example the converters for each edge are chosen to maximize each edge's FOM as defined in (4.2). It is important to note that the results could change considerably if a different optimization goal were chosen. Significant differences can be seen between the best and the worst architectures. In fact, a majority of the architectures are worse than the best in both the quality factor $Q$ and the surface power density $\rho$.

Next, from all possible architectures shown in Fig. 4.1, those that represent the best power density versus system $Q$ factor trade-off are selected and shown in Fig. 4.2. The architectures remaining are those obtained when all architectures with both a lower quality factor and power density are removed. This leaves only a small number of architectures to be considered, and more clearly displays the expected Pareto front representing the achievable power density versus efficiency trade off: a solution that results in higher $Q$ tends to require a larger area for implementation (lower power density).

Finally, it should be noted that an important property of the architecture FOM is that it is not necessarily maximized by maximizing the individual converter's FOM. If an individual converter has a small contribution to the power loss, but a large contribution to the area an equal or lesser 


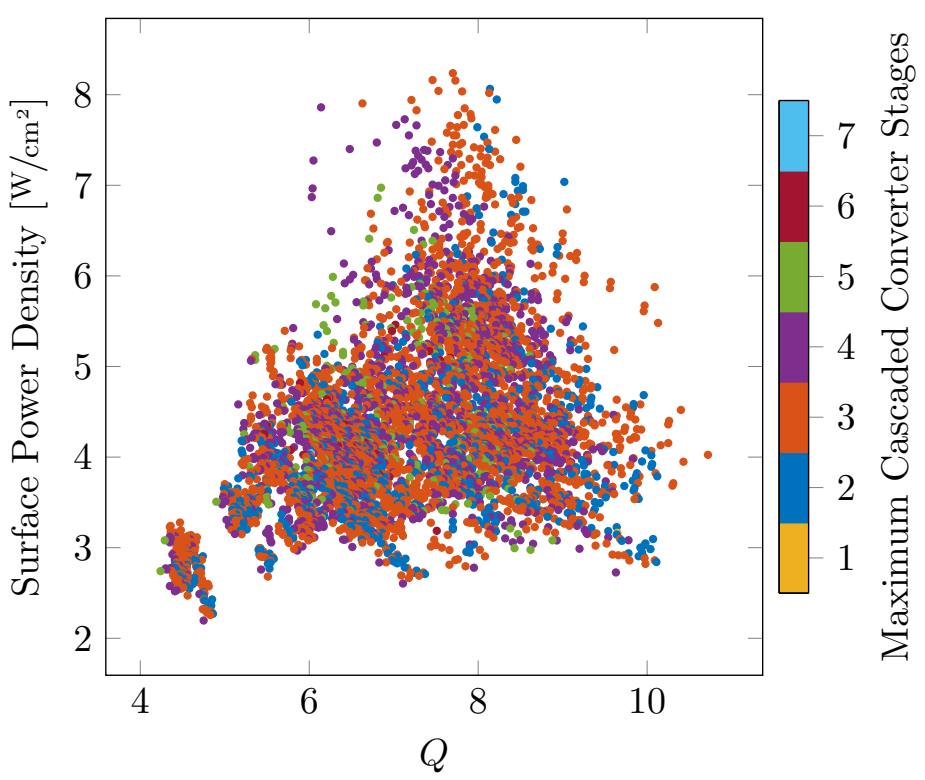

Figure 4.1: Surface power density vs. quality factor $Q=P_{\text {out }} / P_{\text {loss }}$ for all 5040 possible architectures in a test case with seven outputs. In this example the converters for each edge are chosen among the converters that meet the required input and output voltage and current requirements to maximize each edge's FOM as defined in (4.2).

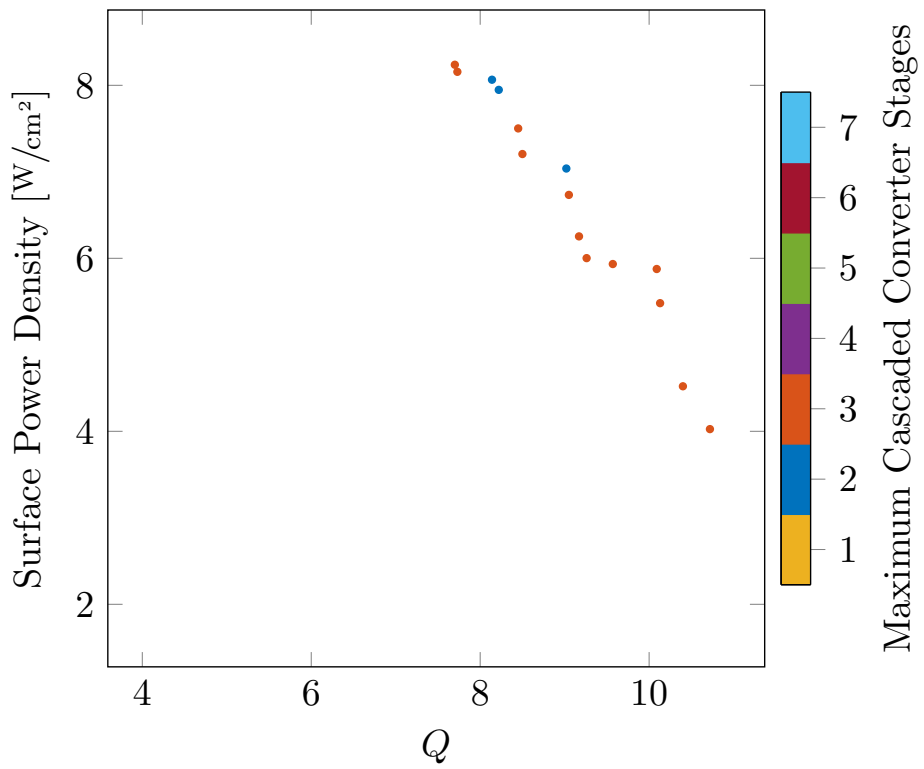

Figure 4.2: Filtered surface power density vs. quality factor $Q=P_{\text {out }} / P_{\text {loss }}$ for the seven-output test case considered in Fig. 4.1. The Pareto front is generated by showing only the solutions from Fig. 4.1 that have a higher quality factor than all other architectures with greater than or equal surface power density. 
converter FOM could result in a higher system FOM given the same arrangement. 


\section{Chapter 5}

\section{Exhaustive Search Optimization}

For the non-convex, discrete optimization problem of designing de distribution architectures the only way to guarantee finding the optimal result is by complete enumeration and evaluation of the full design space. This exhaustive search solution will ensure that the optimal solution is found at the cost of computational expense. The exhaustive search implementation is described in [10], [41] as the Permutation-Graph (PG) Method. A high-level flow chart of the PG method can be seen in Fig. 5.1. The method systematically enumerates and chooses the optimal arrangement for a DC distribution system containing multiple voltage domains. To begin, the optimization procedure finds all possible arrangements of the given voltage domains, it then finds converters to realize the connections in each arrangement, and finally it determines system wide attributes for each

architecture and selects the optimal solution based on user defined goals. Detailed explanations of each step are provided in Section 5.1 and Chapters 3 and 4.

\subsection{Enumeration of Bucking or Boosting Trees}

As shown in Chapter 2 there are multiple design spaces that can be enumerated to find all possible arrangements. As possible graphs and spanning trees both grow quickly an exhaustive enumeration of each of their arrangements quickly becomes intractable. Therefore, the exhaustive search solver will consider only the arrangements in the bucking and boosting trees design space.

Enumeration of all the bucking or boosting trees can be accomplished by finding all permutations of the lists of output voltages below or above the input, respectively, and then working through each list systematically. In the bucking case, the first element in the list is attached to 


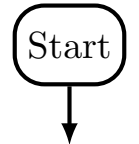

User specifies system requirements

$V_{\text {in }},\left\{V_{\text {out }, i}\right\},\left\{P_{\text {out }, i}\right\}$ and computa-

tional complexity modifiers $n_{\max }, n_{\text {bus }}$

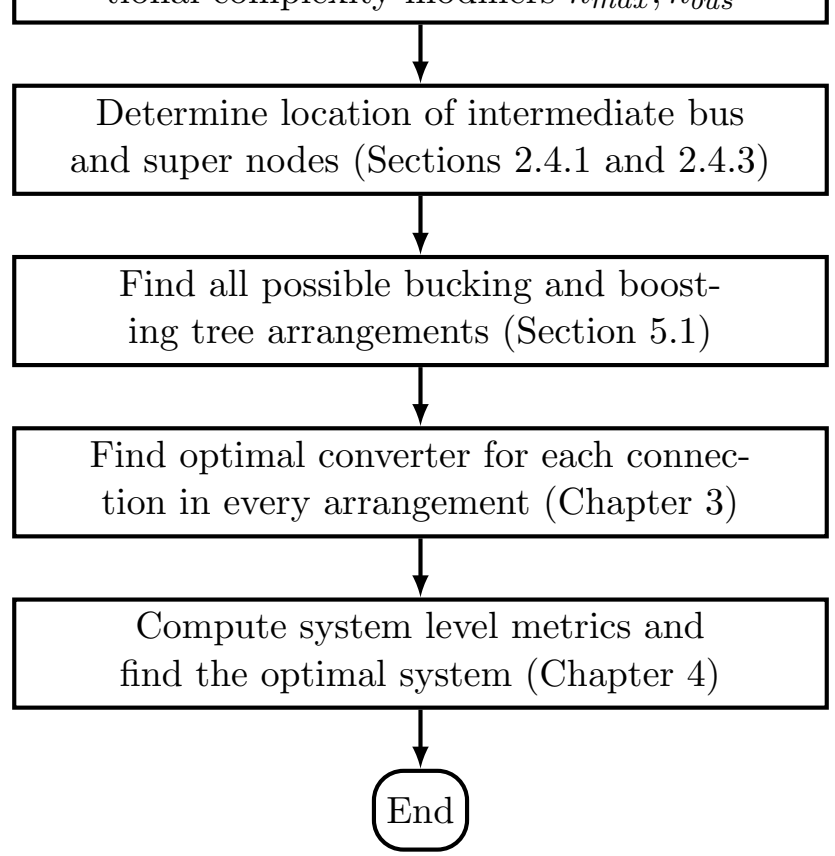

Figure 5.1: Top level flow chart of the permutation graph approach for optimization of dc distribution architectures.

the input voltage in all cases. If the second element is less than the first it is connected to the first, and if not it is also connected to the input. Moving down the remainder of the list, each output is connected to the closest preceding voltage that is of a larger value (or the input if no other option is available). It is from this process of enumerating graphs through permutations that the PG method [41] inherits its name. Simplified pseudocode for this operation can be found in Algorithm 5.1. A similar algorithm may be followed to generate the boosting trees.

To illustrate the algorithm by an example, consider the system in Fig. 1.3 shown in the graph form in Fig. 1.4a. This arrangement is created from the permutation $\{24,5,1,3.3,12\}$. Start by attaching the first element, 24, to the input. The second element, 5, is less than 24 so it is attached to the 24 node. The third element, 1 , is less than 5 so it is attached to the 5 node. The forth element, 3.3, is greater than 1 and less than 5 , so it is attached to the 5 node. The final element, 
12, is greater than 3.3 and 5 , so it is attached to the 24 node. As another example, the arrangement in Fig. 1.4b is generated from the permutation $\{24,12,1,3.3,5\}$.

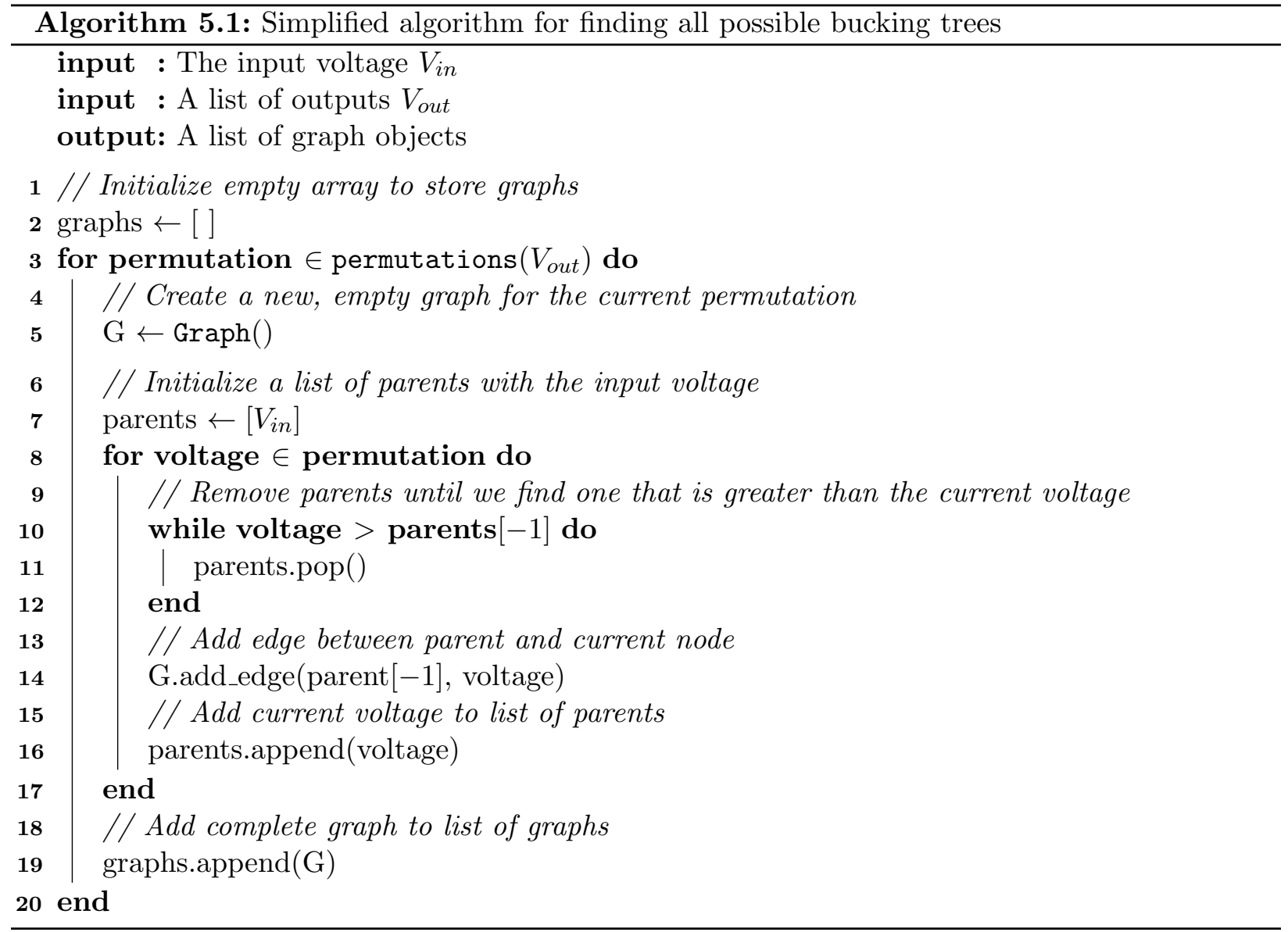

Pseudocode of the complete algorithm that incorporates both super nodes and intermediate bus voltages into the arrangement generation process together with support for boosting trees is provided in Appendix A.

\subsection{Performance Comparison}

To evaluate the performance of the PG method compared to the current state of the art several case studies were carried out. The first is discussed in [10], which compares the TI WEBENCH Power Architect [29] tool with results generated by the PG method using only converters in the TI database. In these test cases a system with a $48 \mathrm{~V}$ input and seven outputs, $40,24,12,5,3.3,1.3$, and $1 \mathrm{~V}$, is considered at various output power levels. The main results are summarized in Fig. 5.2 


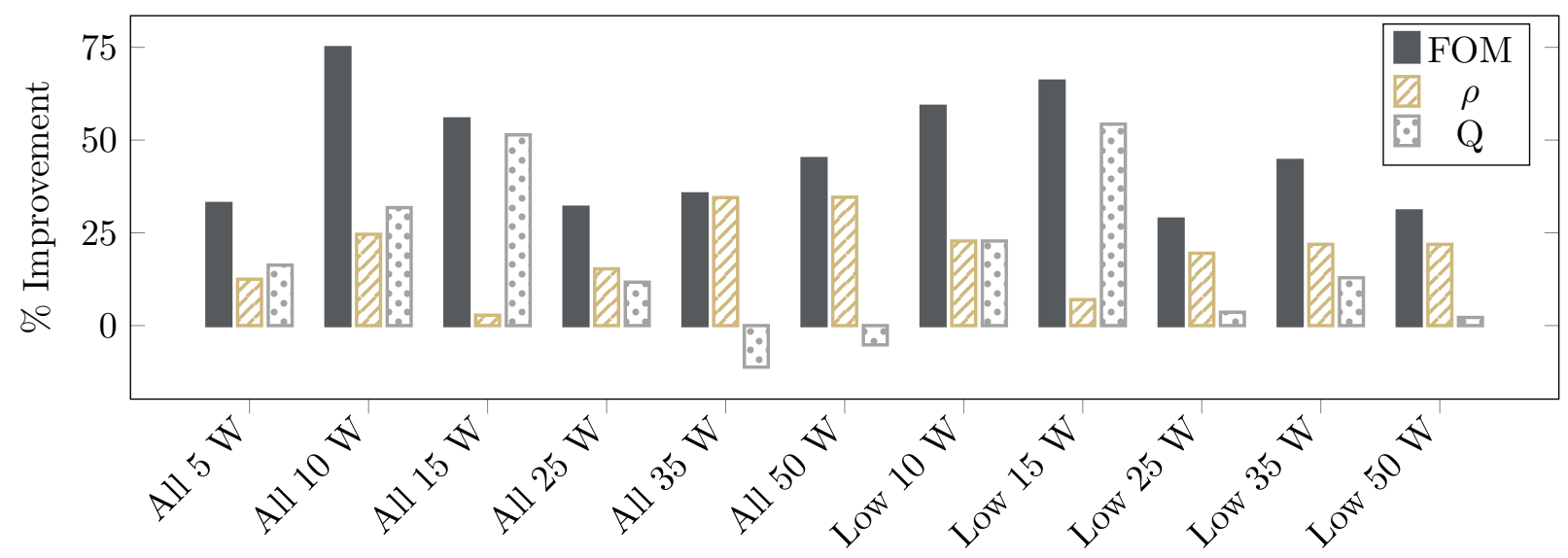

Figure 5.2: Improvements offered by the PG algorithm compared to the results obtained using [29] for different test cases. In each test case the input is $48 \mathrm{~V}$ and the outputs are $40,24,12,5,3.3$, 1.3 , and $1 \mathrm{~V}$. For the "Low" test cases low refers to voltage domains $5 \mathrm{~V}$ and below and the other outputs are $5 \mathrm{~W}$ each. The system FOM is defined in (4.2) as $Q \rho / P_{\text {out }}$

which shows that depending on the test case the PG method produces results between $30 \%$ to $75 \%$ better than those produced by [29] using the FOM described in (4.2). A detailed examination of these results shows that the improvements do not always take the same form and that the optimal result produced by each method can be very similar or quite different, highlighting the need to perform a broad systematic search to achieve the optimal result.

\subsubsection{All Voltages $10 \mathrm{~W}$}

A detailed examination of the individual test cases helps provide insight into how the PG method offers improved performance. Fig. 5.4 shows the arrangements obtained by the two methods and Tables 5.1 and 5.2 compare the converters selected with each method for the case when all voltages are set to $10 \mathrm{~W}$. This case shows the the greatest improvement over [29]. The arrangements for each method are quite similar, with only two nodes repositioned; however, the improvement from one to the other is substantial.

The greatest impact is seen on the 5 and $3.3 \mathrm{~V}$ converters with an increase in FOM of 173 and $97 \%$, respectively, due to the reduction in conversion ratio. The impact of conversion ratio on converter FOM can be seen in Fig. 5.9 where it is clear that a higher conversion ratio results in 


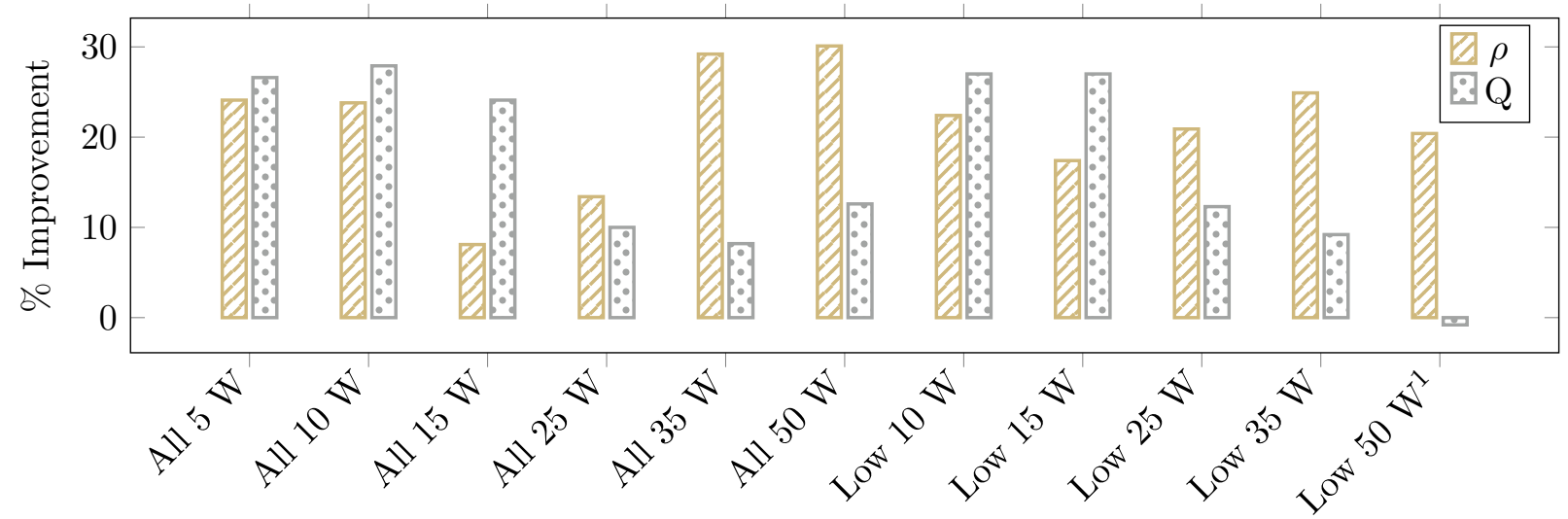

${ }^{1}$ When optimizing for efficiency the WEBENCH Results for the Low $50 \mathrm{~W}$ case are better than the PG method because the WEBENCH solution makes use of a $7 \mathrm{~V}$ pre-regulation node.

Figure 5.3: Improvements offered by the PG method compared to the results obtained using [29] for different test cases when optimizing for efficiency or power density.

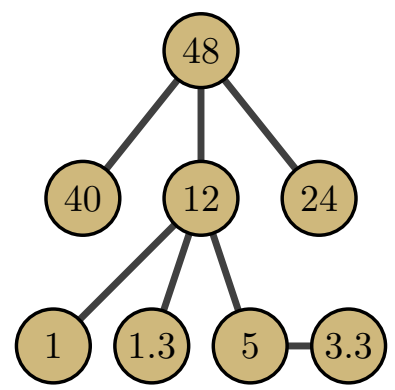

(a)

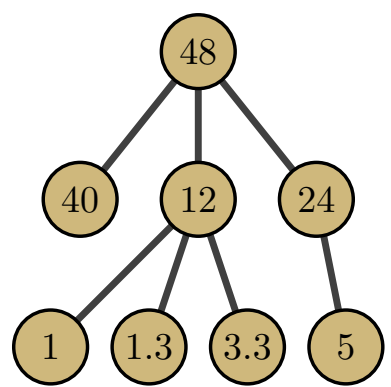

(b)

Figure 5.4: The two arrangements generated for the All Voltages $10 \mathrm{~W}$ Case discussed in Section 5.2.1. $\mathrm{a}$ is for the PG method and $\mathrm{b}$ is for [29].

lower performing converters. A slight increase in FOM for the $12 \mathrm{~V}$ converter due the increase in power processed is also shown. The impact on amount of power processed on FOM can be seen in Fig. 5.6; however, it is not necessarily true that as power increases FOM also increases. There are steps, dips, and occasional downward trends in the curve that may lead to unpredictable results. Fig. 5.6 is created by holding the input and output voltages constant and changing the required power and requesting converters from the WEBENCH tool [29]. For each power level in the sweep the converter with the highest FOM is chosen and plotted. Additionally, tick marks show where the the converter used changed, either by the control IC or the components used. 
Table 5.1: WEBENCH [29] converters for all voltages at $10 \mathrm{~W}$

\begin{tabular}{llllr}
\hline$V_{\text {out }}$ & Part & FOM $\left[1 / \mathrm{dm}^{2}\right]$ & $\eta[\%]$ & Area $\left[\mathrm{mm}^{2}\right]$ \\
\hline \hline 1 & TPS53915 & 315 & 83.80 & 164 \\
1.3 & TPS53915 & 381 & 86.50 & 168 \\
3.3 & TPS53915 & 1048 & 94.20 & 155 \\
5 & LMR23630AP & 685 & 90.50 & 139 \\
12 & LM5145 & 582 & 94.20 & 279 \\
24 & LMR36015A & 717 & 95.80 & 318 \\
40 & LM5008A & 707 & 92.90 & 185 \\
\hline
\end{tabular}

Table 5.2: PG method converters for all voltages at $10 \mathrm{~W}$

\begin{tabular}{llllr}
\hline$V_{\text {out }}$ & Part & FOM $\left[1 / \mathrm{dm}^{2}\right]$ & $\eta[\%]$ & Area $\left[\mathrm{mm}^{2}\right]$ \\
\hline \hline 1 & TPS53319 & 407 & 90.65 & 238 \\
1.3 & TPS53319 & 511 & 89.84 & 173 \\
3.3 & TPS62182 & 2065 & 93.53 & 70 \\
5 & TPS62180 & 1872 & 94.73 & 96 \\
12 & LM5145 & 839 & 95.89 & 278 \\
24 & LM5575-Q1 & 1638 & 95.58 & 132 \\
40 & LM5166 & 4281 & 96.94 & 74 \\
\hline
\end{tabular}

\subsubsection{Low Voltages $15 \mathrm{~W}$ - Others $5 \mathrm{~W}$}

Clearly, the arrangements found by each method are quite similar with only one node repositioned; however, the improvements are substantial. The selection of converters shows why such a change is possible from a small alteration in arrangement. By moving the $5 \mathrm{~V}$ domain's input to the $12 \mathrm{~V}$ node the conversion ratio is halved and an improved converter may be selected. Additionally, as the $48 \mathrm{~V}$ to $24 \mathrm{~V}$ converter must no longer supply the power required by the $5 \mathrm{~V}$ load it's FOM is also able to improve. The $48 \mathrm{~V}$ to $12 \mathrm{~V}$ converter also improves as a result of the arrangement adjustment. It is now processing more power and is able to achieved a higher efficiency, this results in an improved FOM despite the required increase in footprint. 


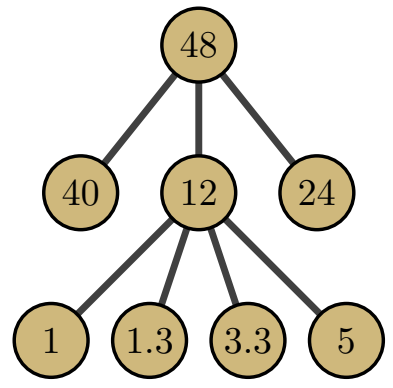

(a)

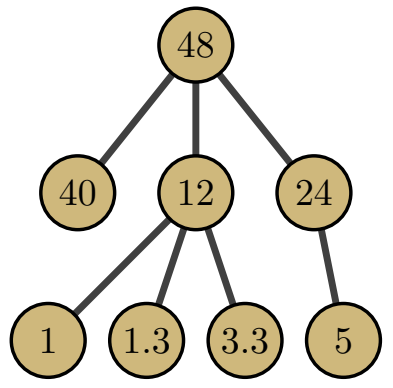

(b)

Figure 5.5: The two arrangements generated for the Low Voltages $15 \mathrm{~W}$ - Others $5 \mathrm{~W}$ Case discussed in Section 5.2.2. a is for the PG method and b is for [29].

Table 5.3: WEBENCH [29] converters for low voltages at $15 \mathrm{~W}$

\begin{tabular}{llllr}
\hline$V_{\text {out }}$ & Part & FOM $\left[1 / \mathrm{dm}^{2}\right]$ & $\eta[\%]$ & Area $\left[\mathrm{mm}^{2}\right]$ \\
\hline \hline 1 & LM25145 & 190 & 83.50 & 265 \\
1.3 & TPS40345 & 340 & 87.90 & 216 \\
3.3 & TPS53915 & 1120 & 94.10 & 142 \\
5 & LMR33630B & 810 & 89.60 & 106 \\
12 & LM5145 & 672 & 94.50 & 277 \\
24 & LMR36015A & 720 & 95.80 & 318 \\
40 & LM5008A & 1340 & 94.50 & 128 \\
\hline
\end{tabular}

Table 5.4: PG method converters for low voltages at $15 \mathrm{~W}$

\begin{tabular}{llllr}
\hline$V_{\text {out }}$ & Part & FOM $\left[1 / \mathrm{dm}^{2}\right]$ & $\eta[\%]$ & Area $\left[\mathrm{mm}^{2}\right]$ \\
\hline \hline 1 & LM27403 & 340 & 91.34 & 314 \\
1.3 & TPS53319 & 480 & 91.92 & 238 \\
3.3 & TPS53915 & 1170 & 94.19 & 139 \\
5 & TPS62180 & 2080 & 95.24 & 96 \\
12 & LM5145 & 820 & 96.75 & 365 \\
24 & LM5166 & 1450 & 95.04 & 132 \\
40 & LM5166 & 4440 & 97.12 & 76 \\
\hline
\end{tabular}

\subsubsection{All Voltages $25 \mathrm{~W}$}

For the test case with all voltage domains providing $25 \mathrm{~W}$ to their respective loads the two arrangements are shown in Fig. 5.10 and the converters for each are shown in Tables 5.5 and 5.6. In 


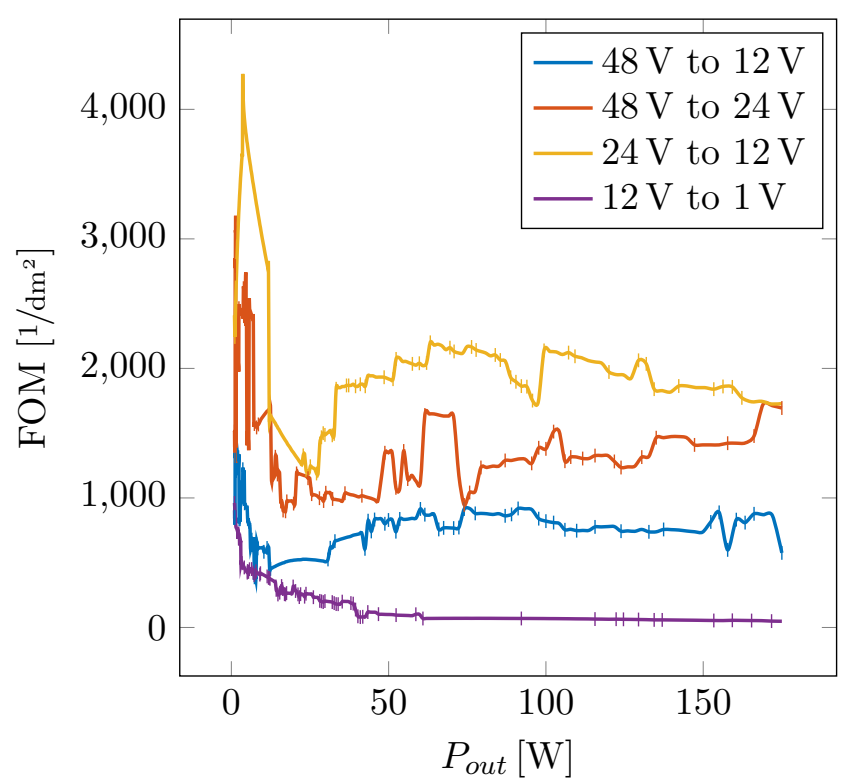

Figure 5.6: Power Vs. Figure of Merit for two converters with different output voltages. These curves are shown for converters that are available from the WEBENCH Tool. Each tick mark shows a point where a different converter is optimally chosen from the WEBENCH results [29].

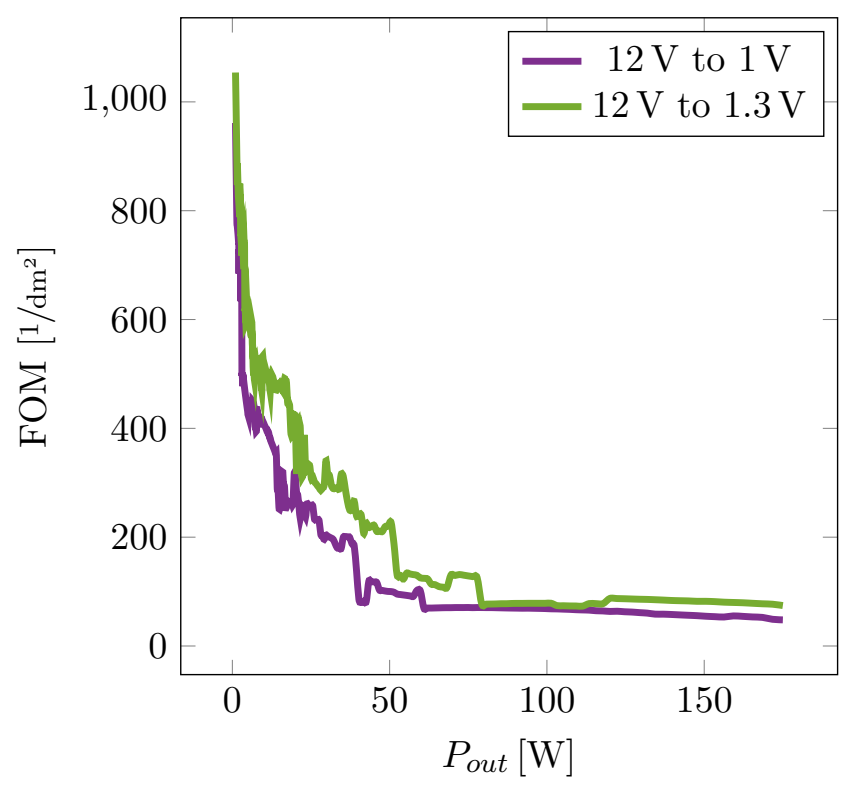

Figure 5.7: Power Vs. Figure of Merit for two converters with different output voltages. 


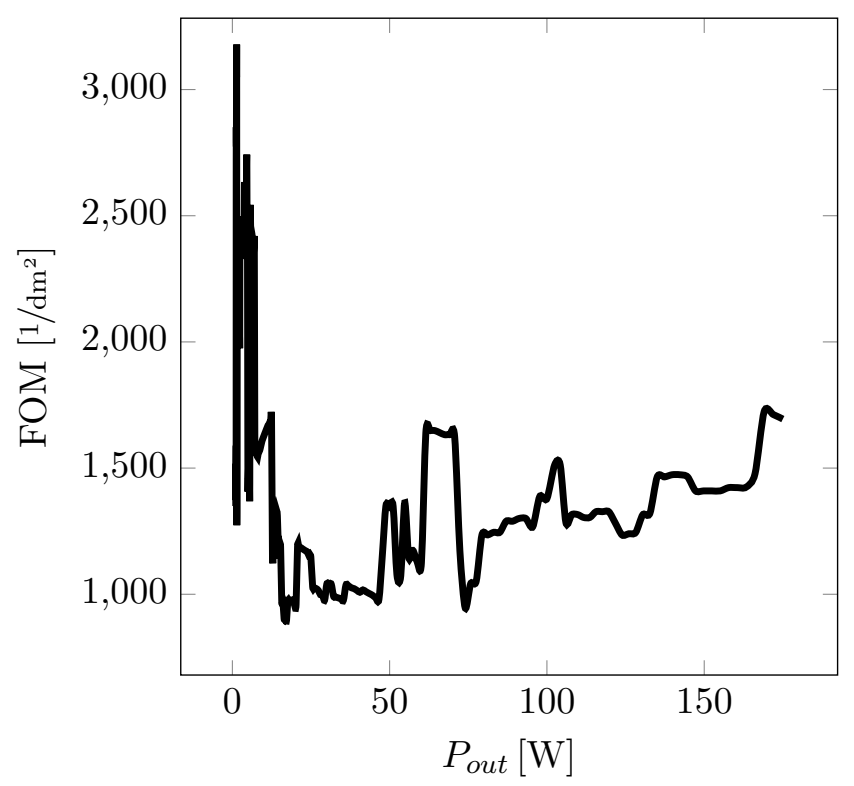

Figure 5.8: Power Vs. Figure of Merit for a $48 \mathrm{~V}$ to $24 \mathrm{~V}$ converter.

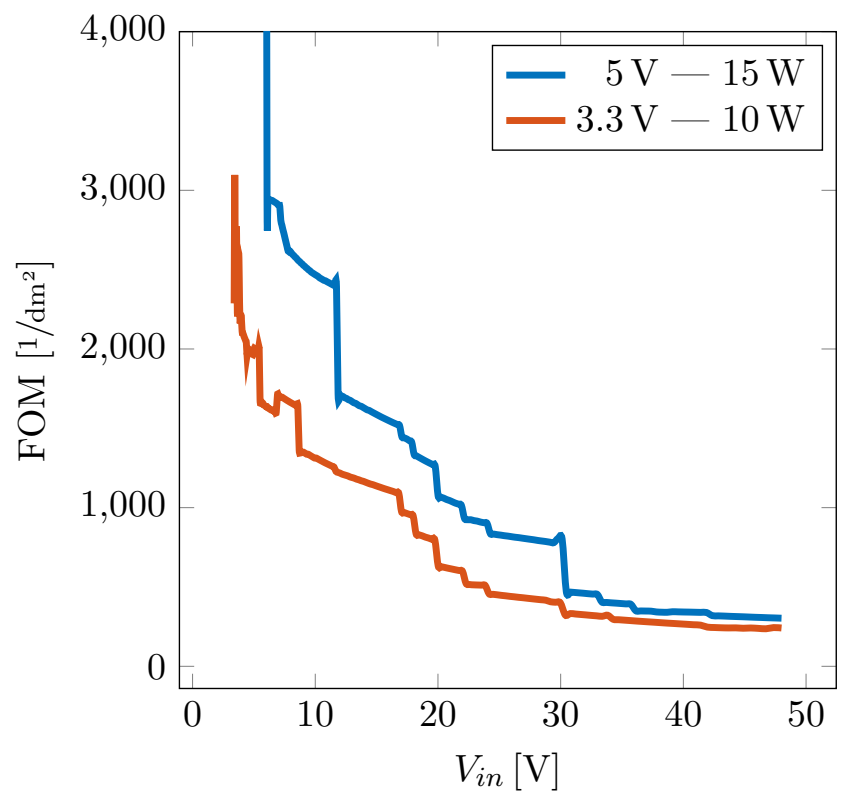

Figure 5.9: Input Voltage Vs. Figure of Merit for two converters with different output voltages and power levels. These curves are shown for converters that are available from the WEBENCH Tool [29]. 


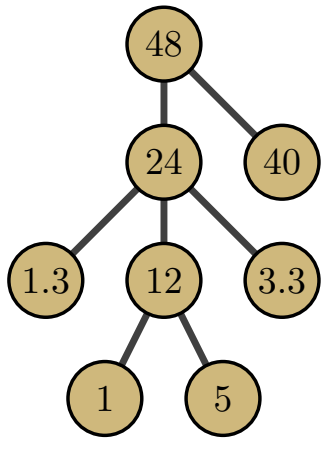

(a)

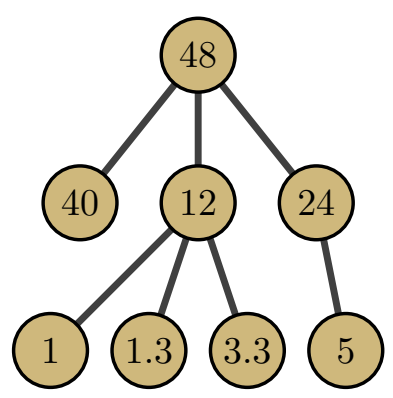

(b)

Figure 5.10: The two arrangements generated for the All Voltages $25 \mathrm{~W}$ Case discussed in Section 5.2.3. $\mathrm{a}$ is for the PG method and $\mathrm{b}$ is for [29].

this case the difference in optimum arrangement found by the two methods is much more dramatic. A complex three-layer structure is present in the PG method solution, which counter intuitively does not attempt to minimize conversion ratios. Note how the $5 \mathrm{~V}$ node is attached to the $12 \mathrm{~V}$ node while the lower 1.3 and $3.3 \mathrm{~V}$ nodes are attached to the $24 \mathrm{~V}$ node. However, looking again at Figs. 5.6 and 5.9 a decrease in FOM for the $24 \mathrm{~V}$ to $12 \mathrm{~V}$ converter can be seen when the power increases from $80 \mathrm{~W}$ with the arrangement in Fig. 5.10a to $134 \mathrm{~W}$ for the arrangement with all the lower voltages feeding off the $12 \mathrm{~V}$ node. This decrease does not make up for the increase seen by reducing the conversion ratio of the 1.3 and $3.3 \mathrm{~V}$ converters. Fig. 5.9 is created in a similar manner to Fig. 5.6 with output power and output voltage being held constant and sweeping input voltage.

This case helps demonstrate the need to check all arrangements as the way the converter's performance changes with changing inputs and outputs is non-linear and difficult to predict. These non-linear characteristics are due to the discrete nature of commercially available converter configurations and devices. Improvements in automated converter design could reduce these effects in the future; however, when using commercially available devices there will always be some steps at the boundary between two, in addition to the steps between converter configurations. 
Table 5.5: WEBENCH [29] converters for all voltages at $25 \mathrm{~W}$

\begin{tabular}{llllr}
\hline$V_{\text {out }}$ & Part & FOM $\left[1 / \mathrm{dm}^{2}\right]$ & $\eta[\%]$ & Area $\left[\mathrm{mm}^{2}\right]$ \\
\hline \hline 1 & TPS40345 & 200 & 88.20 & 372 \\
1.3 & LM25145 & 300 & 90.20 & 304 \\
3.3 & TPS53915 & 960 & 94.20 & 170 \\
5 & TPS40170 & 610 & 93.00 & 217 \\
12 & LM5145 & 740 & 96.00 & 323 \\
24 & LM5088-2 & 1360 & 98.00 & 359 \\
40 & LM76002 & 940 & 97.20 & 371 \\
\hline
\end{tabular}

Table 5.6: PG method converters for all voltages at $25 \mathrm{~W}$

\begin{tabular}{llllr}
\hline$V_{\text {out }}$ & Part & FOM $\left[1 / \mathrm{dm}^{2}\right]$ & $\eta[\%]$ & Area $\left[\mathrm{mm}^{2}\right]$ \\
\hline \hline 1 & TPS543C20 & 230 & 85.68 & 258 \\
1.3 & LM5145 & 280 & 89.58 & 306 \\
3.3 & LM5145 & 740 & 94.99 & 255 \\
5 & TPS53915 & 1690 & 95.91 & 139 \\
12 & LM25145 & 2090 & 98.04 & 239 \\
24 & LM5145 & 1730 & 98.56 & 396 \\
40 & LM5576 & 3300 & 98.50 & 199 \\
\hline
\end{tabular}

\subsubsection{Comparison with Prior Work}

In addition to the comparison with the commercial tool, WEBENCH [29], a comparison is performed against the PD3T tool presented in [26], [27]. In this test case the loads presented in Table I of [27] are combined and subsequently processed by the PG method. As several of the voltage domains in the considered test case are higher than the input, the boost arrangement was assumed to optimally have a depth of one and the converters for these loads are found separately. Additionally, as insufficient details are provided for the converters used in [27], it is assumed that the area estimates were accurate, and so no area scale factor is applied. The area of the solutions found using the PG method is scaled up by the larger of the factors discussed in Section 8.1, i.e., by approximately a factor of two. Fig. 5.11 and Table 5.7 shows the results found with the PD3T tool compared to the PG method. Comparing the highest FOM between the two methods shows an 


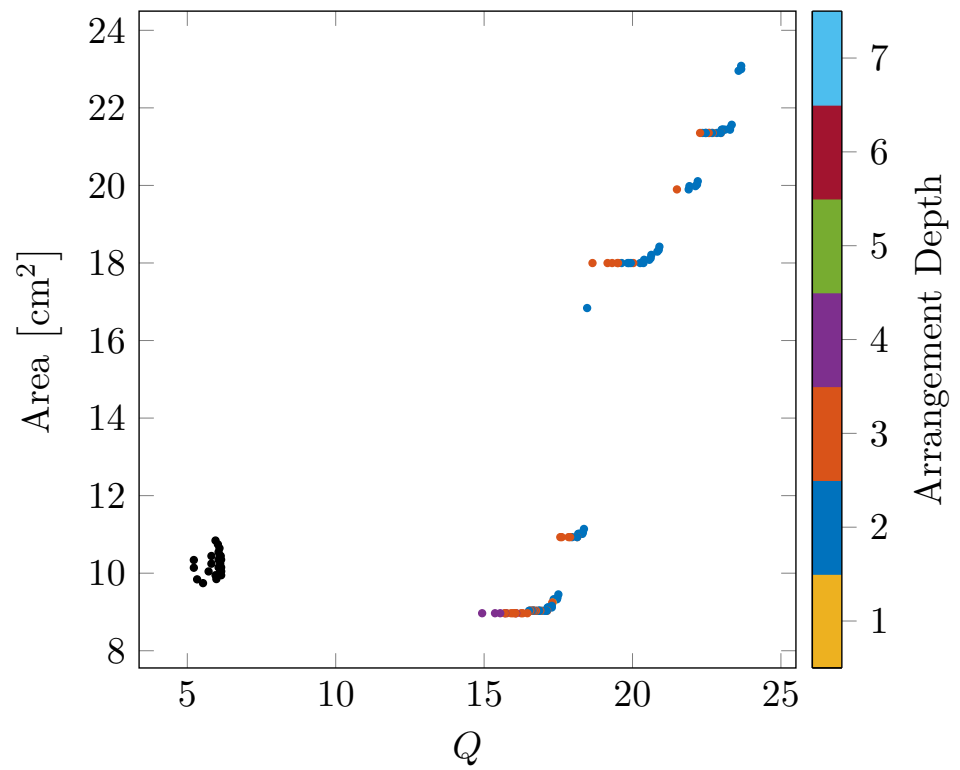

Figure 5.11: $Q$ vs. Area Plot for the test case in [27]. Black dots represent results from PD3T [27] and the colored dots are those from the PG method.

increase in FOM of more than $200 \%$. There are a few potential reasons for the improvement shown with the PG method, the first is a dramatic increase in the pool of available converters. The tool in [27] used less than forty converters to construct its solutions, but the PG method makes use of a pool of thousands of converters. Additionally, the PG method looks at all possible arrangements while the PD3T tool looks at only those heuristically selected.

Table 5.7: PD3T [27] results for test case presented in [27]

\begin{tabular}{rlllrr}
\hline \multicolumn{2}{c}{$\eta[\%]$} & \multicolumn{2}{c}{ Area $\left[\mathrm{mm}^{2}\right]$} & \multicolumn{2}{c}{ FOM $\left[1 / \mathrm{dm}^{2}\right]$} \\
{$[27]$} & PG & {$[27]$} & PG & {$[27]$} & PG \\
\hline 86.0 & 94.5 & 995 & 903 & 61.7 & 189.6 \\
86.0 & 94.5 & 1005 & 903 & 61.1 & 189.5 \\
85.9 & 94.5 & 1005 & 912 & 60.8 & 189.6 \\
\hline 84.2 & 94.0 & 984 & 5596 & 54.2 & 27.8 \\
83.9 & 93.6 & 1014 & 5554 & 51.5 & 26.4 \\
83.9 & 93.6 & 1034 & 5554 & 50.5 & 26.4 \\
\hline
\end{tabular}




\section{Chapter 6}

\section{Descent}

The systematic exhaustive search approach described in the previous chapters works well for relatively small systems that contain seven or fewer outputs. However, as the number of required outputs $\left(n_{o}\right)$ increases, the number of possible arrangements of dc-dc converters increases factorially, as shown in Fig. 6.1. In systems with nine or more outputs, such as in systems containing certain processors or FPGAs [42]-[44], finding a solution becomes intractable. To address this problem, several heuristics are proposed in Section 2.4 to shrink the design space, so that larger problems may still be solved using the exhaustive-search method. However, these design space shrinking heuristics inevitably lead to suboptimal designs, and the impact on the system performance is difficult to characterize.

In order to find an optimal architecture for large systems a new method of searching the design space is desirable. In [41] the system is described as a directed graph, as shown in Fig. 1.4, where the nodes in the graph represent voltage domains and the edges represent dc-dc converters. The optimal solution is the so called, "minimum spanning arborescence." Efficient algorithms [45][47] have existed since the 1960s for finding the minimum spanning arborescence; however, these algorithms do not apply directly because of the power electronics nature of the problem. For the spanning arborescence sought in this work the edge weights are not constant, but change depending on what connections are made after a particular edge. As an example of this problem see the two possible arrangements in Fig. 1.4. In both Fig. 1.4a and Fig. 1.4b there is an edge (i.e. a converter) between the $24 \mathrm{~V}$ node and the $12 \mathrm{~V}$ node. In Fig. 1.4a this converter supplies only the power needed for the $12 \mathrm{~V}$ node's output; however, in Fig. 1.4b the converter must supply the power for 


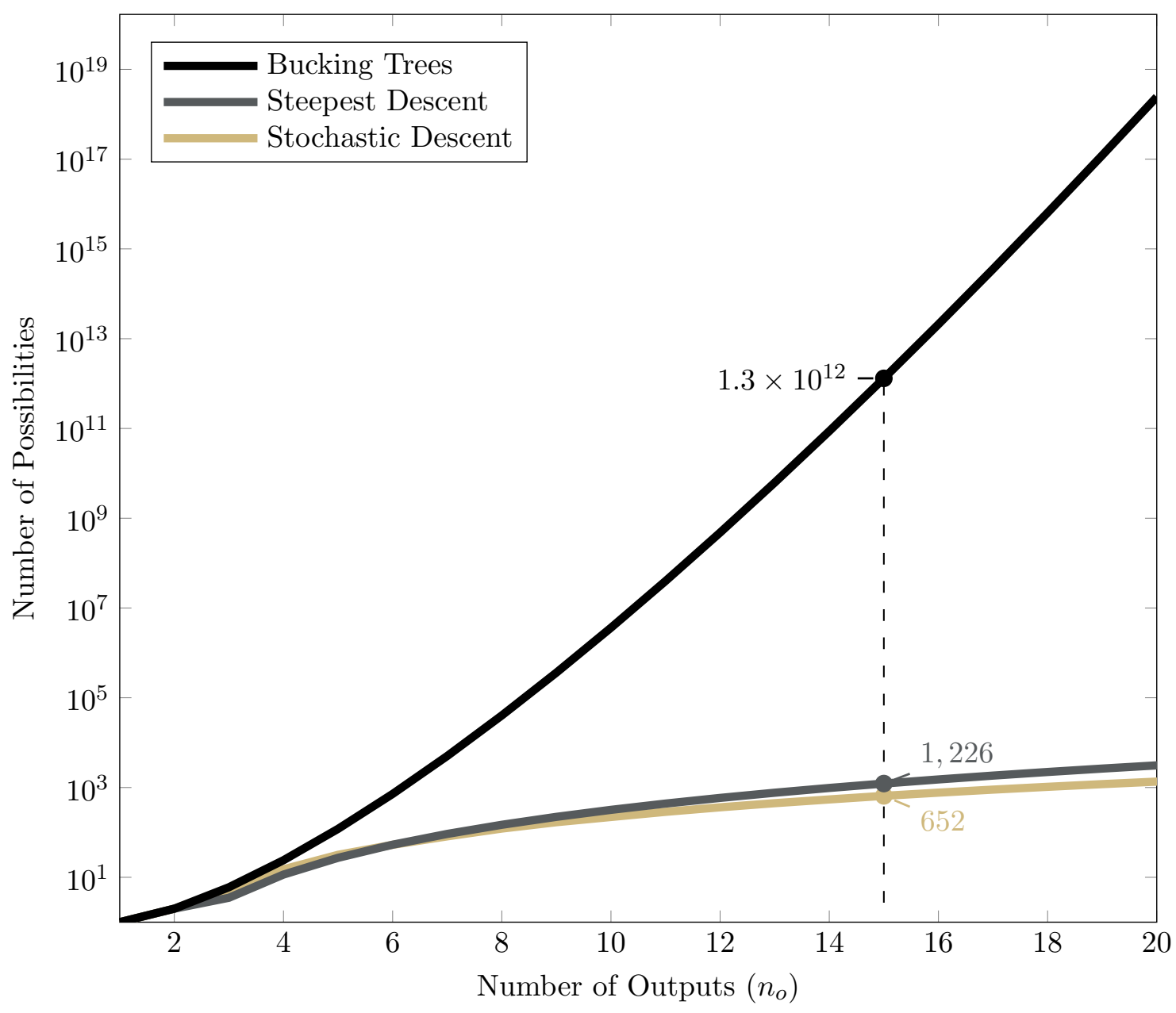

Figure 6.1: Number of possible bucking trees and approximation of number of trees evaluated using steepest descent and stochastic descent as functions of the number of outputs $n_{o}$. Marks indicate the total number of possibilities of each type for $n_{o}=15$. It is clear that steepest descent offers a significant reduction in the number of trees that must be evaluated compared to an exhaustive search and stochastic descent offers further reductions from there.

the $12,5,1$, and $3.3 \mathrm{~V}$ nodes' outputs. This will undoubtedly change the cost of the edge regardless of the metric used.

To design large dc distribution systems efficient optimization procedures are required. In the next two chapters two such techniques are discussed: The first based on descent in Chapter 6 and the second based on simulated annealing in Chapter 7.

This chapter is structured in four sections: first the design space is described in Section 6.1, then steepest descent and stochastic descent algorithms are presented in Sections 6.2 and 6.3, and 


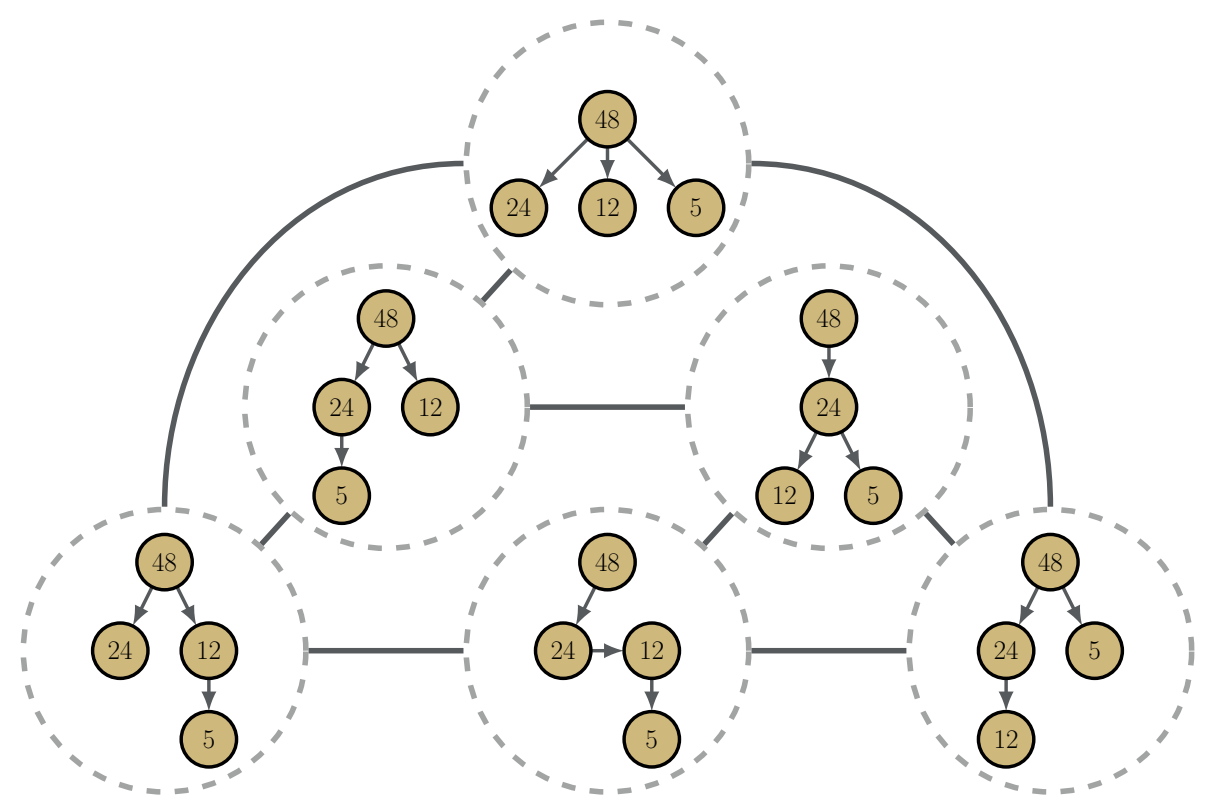

Figure 6.2: Design space graph for a system with three outputs. Each possible arrangement is represented as a node in the graph. The nodes are connected to every other node whose corresponding arrangement differs by only one edge.

finally Section 6.4 presents a series of case studies to compare the performance of descent based methods with the state-of-the-art.

\subsection{Design Space Structure}

The design space in this problem is inherently discrete; each node selects one input from a finite list of possibilities. In Chapter 5 the problem is solved in an exhaustive search manner with all possibilities enumerated and evaluated. However, in this work the optimization procedure aims to move through the design space and therefore the design space's structure is an important factor in the procedure's performance. If the design space is too discrete there will be large changes in performance at every step in the optimization procedure. Therefore, the design space should be constructed such that movement from one solution to another produces a minimally changed system.

To meet these requirements the design space is constructed as a densely connected graph of possible arrangements as in Fig. 6.2. Each node in this graph will correspond to a possible 


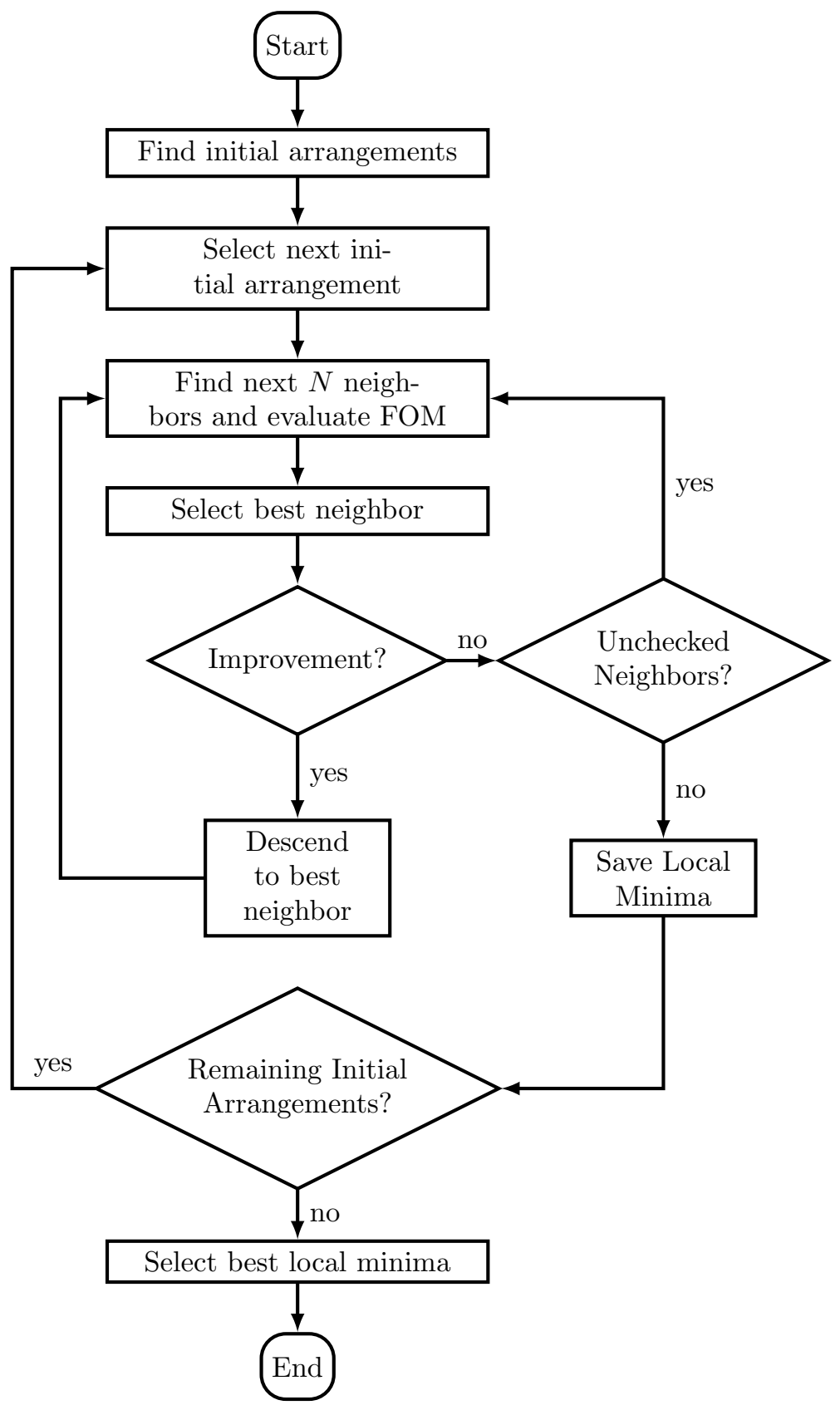

Figure 6.3: Flowchart for operation of stochastic descent and steepest descent when is $N$ is set to all possible neighbors. Initial arrangements are chosen randomly, but they must be feasible. 
arrangement of the system's output voltages. The edges within the design space graph connect arrangements that are different by only one edge.

\subsubsection{Size of the Design Space}

As each node in the design space represents a single arrangement their will be $n_{o}$ ! nodes as shown in Section 2.3. To determine the number of edges connected to each node, i.e. the number of neighbors for each arrangement consider an arbitrary arrangement for a system with $n_{o}$ outputs. Using the same monotonic conversion assumptions as in Chapter 2 for the highest output voltage there are no alternate input nodes as it may only be supplied by the input. For the second highest output voltage there is one possible alternate input node as it may be connected to the input or the highest output. This pattern continues to the lowest output voltage which has $n_{o}-1$ alternate input nodes (The input plus every other output minus its current supply node.) The number of neighbors is the number of arrangements possible with only one change, so the total number of neighbors is the sum of the number of alternate supply nodes for each node in the system

$$
n_{\text {neighbors }}=\sum_{n=1}^{n_{o}} n_{o}-n=\frac{\left(n_{o}-1\right) n_{o}}{2} .
$$

In addition to the number of neighbors for each arrangement in the design space it is also of interest how many arrangements there are at every possible distance from the optimal, where distance is defined as the minimum number of steps to move from the given arrangement to the optimal. To begin we will again consider the number of arrangements one step away from the optimal, i.e. the number of neighbors. Slight manipulation of (6.1) produces

$$
N_{1}=\sum_{n=1}^{n_{o}-1} n .
$$

Now consider the number of arrangements exactly two steps away from the optimal solution. In this case there are two nodes with a parent different from the optimal solution. If the first node perturbed from the optimal is the second highest output then it has just one possible alternative parent (it must be connected to either the input or the highest output.) If the second node perturbed is the third highest output it has two possible alternates for a total of two possible arrangements 
with two perturbations involving the second and third highest outputs. The forth has three, the fifth four, and so on until the lowest output has $n_{o}-1$ possible alternate parents for $n_{o}-1$ possible arrangements with two perturbations involving the second highest and the lowest outputs. Now consider if the first node perturbed is the third highest node. It has two possible alternates and if the second node perturbed is the fourth highest, each of those perturbations can be perturbed in three different ways for six possible arrangements two perturbations from the optimal involving the third and forth highest outputs. The full list of possible arrangements associated with each pair of nodes is

$$
\begin{gathered}
V_{1}: 1 \overbrace{}^{V_{2}}+\overbrace{3}^{V_{3}}+\cdots+\overbrace{n_{o}-1}^{V_{4}}) \\
V_{3}: 2(\overbrace{3}^{V_{4}}+\overbrace{4}^{V_{5}}+\cdots+\overbrace{n_{o}-1}^{V_{n_{o}}}) \\
\vdots \\
V_{n_{o}-1}:\left(n_{o}-2\right)(\overbrace{n_{o}-1}^{V_{n_{o}}})
\end{gathered}
$$

Where $V_{i}$ is the $i$ th highest output. Compacting each row and combining gives

$$
N_{2}=\overbrace{\sum_{n=2}^{n_{o}-1} 1 n}^{V_{2}}+\overbrace{\sum_{n=3}^{n_{o}-1} 2 n+\cdots+\overbrace{\sum_{n=n_{o}-1}^{n_{o}-1}\left(n_{o}-2\right) n}^{V_{3}}}^{V_{n_{o}}}
$$

which can be furthered simplified to

$$
N_{2}=\sum_{m=1}^{n_{o}-2} \sum_{n=m+1}^{n_{o}-1} m n .
$$

Now consider the number of arrangements exactly three steps from the optimal solution where three of the nodes in each perturbed arrangement has three nodes with a suboptimal parent. Following a similar logic as with the two step enumeration the number of perturbed arrangements associated with each first perturbed node is

$$
V_{2}: 1(\overbrace{2(\underbrace{3}_{V_{4}}+\underbrace{4}_{V_{5}}+\cdots+\underbrace{\left(n_{o}-1\right)}_{V_{n_{o}}})}^{V_{3}}
$$




$$
\begin{aligned}
& +\overbrace{3(\underbrace{4}_{V_{5}}+\underbrace{5}_{V_{6}}+\cdots+\underbrace{\left(n_{o}-1\right)}_{V_{n_{0}}})}^{V_{4}} \\
& +\overbrace{\left(n_{o}-2\right) \underbrace{V_{n_{o}-1}^{\left(n_{o}-1\right)}}_{V_{n_{o}}})}^{V_{n}} \\
& V_{3}: 2(\overbrace{3(\underbrace{4}_{V_{5}}+\underbrace{5}_{V_{6}}+\cdots+\underbrace{\left(n_{o}-1\right)}_{V_{n_{o}}})}^{V_{4}} \\
& +\overbrace{4(\underbrace{5}_{V_{6}}+\underbrace{6}_{V_{7}}+\cdots+\underbrace{\left(n_{o}-1\right)}_{V_{n_{o}}})}^{V_{5}} \\
& +\overbrace{\left(n_{o}-2\right) \underbrace{\left.V_{n_{o}} n_{o}-1\right)}_{V_{n_{o}}})}^{V_{0}} \\
& \vdots \\
& V_{n_{o}-2}:\left(n_{o}-3\right)(\overbrace{\left(n_{o}-2\right) \underbrace{V_{n_{o}-1}^{\left.n_{o}-1\right)}}_{V_{n_{o}}}}^{V_{0}})
\end{aligned}
$$

which can be simplified to

$$
N_{3}=\sum_{p=1}^{n_{o}-3} \sum_{m=p+1}^{n_{o}-2} \sum_{n=m+1}^{n_{o}-1} p m n .
$$

The pattern from (6.2), (6.5) and (6.7) continues until considering arrangements $n_{o}-1$ steps from the optimal which is the maximum. Here the expression is

$$
N_{n_{o}-1}=\sum_{i_{1}=1}^{1} \sum_{i_{2}=i_{1}+1}^{2} \ldots \sum_{i_{n_{o}-1}=n_{o}-1}^{n_{o}-1} \prod_{j=1}^{n_{o}-1} i_{j}=\left(n_{o}-1\right) !
$$

An arbitrary recursive implementation of these expressions is shown in Algorithm 6.1.

\subsection{Steepest Descent}

Steepest descent represents the most straightforward way to move through the design space as defined in Section 6.1. First an arrangement in the design space is selected randomly and its FOM is evaluated based on the user design goals as in Chapter 4. Then all of the design space neighbors are found and evaluated. Once all neighbors are evaluated the best neighbor is selected and the algorithm "descends" to that node in the design space. If there is no neighbor better 


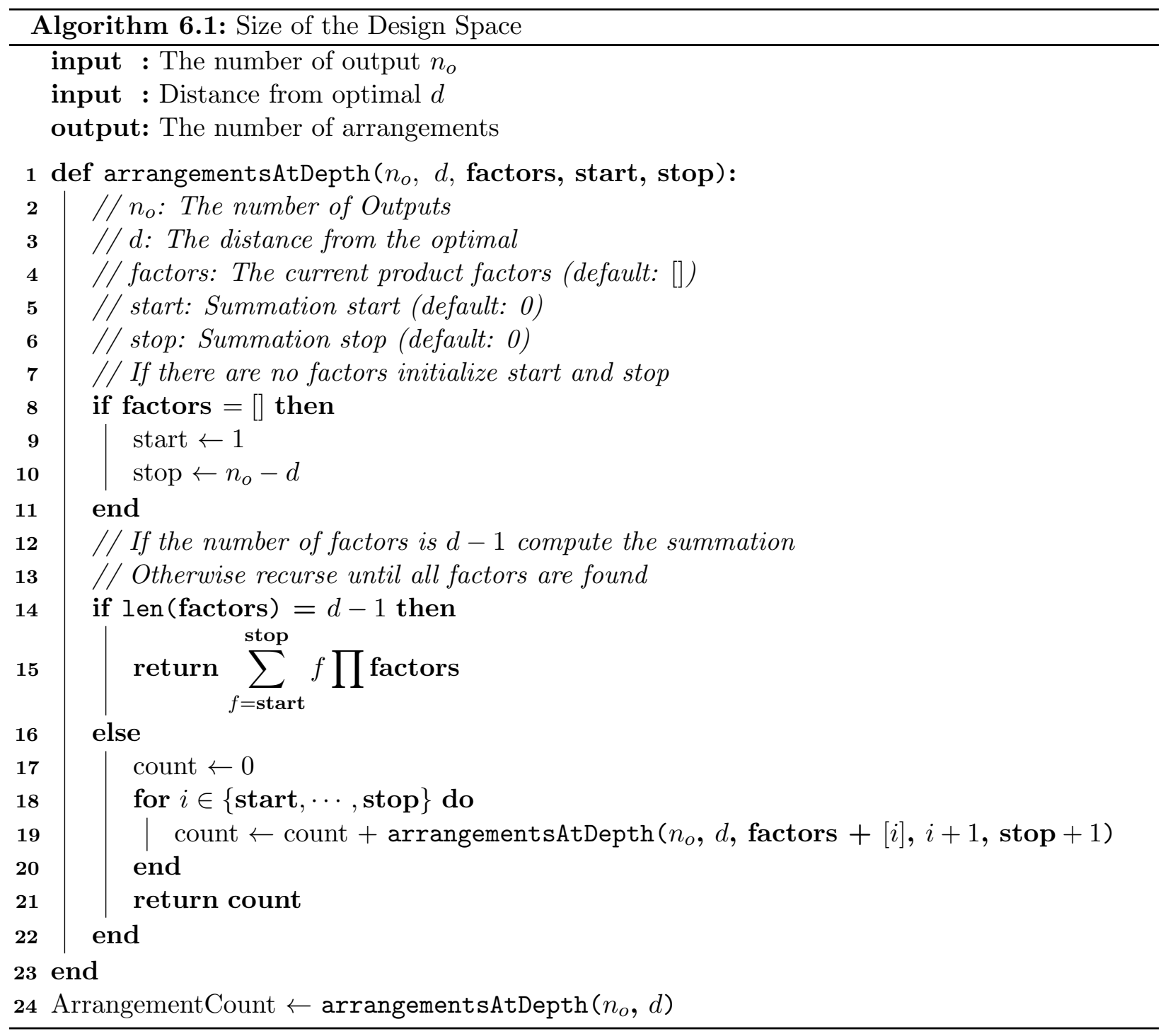

than the current arrangement the arrangement is a local minima. As this problem is discrete and non-convex there is no global optimality guarantee, so the process may be repeated for multiple random starting points to help avoid local minima.

\subsubsection{Steepest Descent Scaling}

The number of arrangements that must be evaluated to find the solution in steepest descent is the number of neighbors for each arrangement multiplied by the number of descent steps before reaching a global optimal

$$
n_{\text {eval }}=n_{\text {neighbors }} n_{\text {steps }} .
$$


As shown in (6.1) the number of neighbors scales with $n_{o}^{2}$. Additionally, if the ideal path through the design space is chosen, there will be at most $n_{o}-1$ descent steps (each outputs supply node changing once to its optimal value.) This adds a further scaling component of approximately $n_{o}$ as it may take more or less steps depending on the starting arrangement and the optimization goal.

For a more precise computation of the number of arrangements which must be evaluated the analysis from Section 6.1 may be used. For each $n_{o}$ the total number of arrangements $n$ steps from the optimal, $N_{n}$ can be computed using (6.2), (6.5), (6.7) and (6.8). The probability that the starting arrangement will start $n$ steps from the input can then be computed by dividing $N_{n}$ by the total number of arrangements, $n_{o}$ !

$$
P_{n}=\frac{N_{n}}{n_{o} !}
$$

Multiplying $P_{n}$ by $n$ and summing over all $n$ gives the average number of steps and multiplying by $N_{1}$ gives the total number of evaluated arrangements on average

$$
n_{\text {eval }, a v g}=N_{1} \sum_{n=0}^{n_{o}-1} P_{n} n .
$$

Fig. 6.1 shows $n_{\text {eval,avg }}$ for steepest descent and stochastic descent plotted against the number of bucking trees, which shows a considerable reduction in the computational expense required for steepest descent compared to an exhaustive search. This analysis assumes that after evaluating all neighbor architectures the best neighbor architecture is in fact closer to the global optimal solution than the current solution. While it is always true that the best neighbor will have an improved cost compared to the current solution, there is no guarantee that it will share more edges with the optimal solution than the current architecture due to the non-convex design space.

\subsection{Stochastic Descent}

Stochastic descent is a generalization of steepest descent where neighbors are evaluated $N$ at a time and descent occurs as soon as an improved neighbor is found. A flow chart that describes both stochastic and steepest descent algorithms is shown in Fig. 6.3. 


\subsubsection{Stochastic Descent Scaling}

To approximate the number of arrangements evaluated using stochastic descent consider again the analysis in Section 6.1. The probability that the starting arrangement is $n$ steps from the optimal can be computed using (6.10). Then, given that an arrangement is $n$ steps from the optimal, there exists $n$ arrangements $n-1$ steps from the optimal that may be reached in a single perturbation from the current arrangement. This is because there are $n$ nodes with the wrong parent and each of them may be changed to the correct parent to be $n-1$ steps from the optimal. The total number of neighbors can also be computed with (6.2). With this information the probability, $P_{d}$, that given $n_{s, n}$ samples of the current arrangements neighbors a arrangement $n-1$ steps from the optimal will be found can be computed with a hypergeometric distribution [48]

$$
P_{d}=1-\frac{\left(\begin{array}{c}
n \\
k
\end{array}\right)\left(\begin{array}{c}
N_{1}-n \\
n_{s, n}-k
\end{array}\right)}{\left(\begin{array}{c}
N_{1} \\
n_{s, n}
\end{array}\right)}
$$

where $k=0$ is the number of observed arrangements $n-1$ steps from the optimal and $n_{s, n}$ is the number of evaluated arrangements. By choosing a desired probability, $P_{d}$, and solving for $n_{s, n}$ the number of arrangements that must be evaluated to find an arrangement $n-1$ steps from the optimal can be determined. Next, the number of arrangements that must be evaluated to find the optimal starting from distance $n$ can be computed as

$$
n_{\text {eval }, n}=n_{s, n}+n_{\text {eval }, n-1}
$$

where $n_{\text {eval, } 0}=N_{1}$ as once the optimal is reached all neighbors must be evaluated to ensure it is the minima.

Finally, the average number of arrangements that must be evaluated to reach to optimal for a system with $n_{o}$ outputs can be computed as

$$
n_{\text {eval }, \text { sto }}=\sum_{n=0}^{n_{o}-1} P_{n} n_{\text {eval }, n}
$$

Fig. 6.1 shows the number of arrangements that must be evaluated to reach a $95 \%$ probability that an arrangement closer to the optimal was sampled before descending. 
Table 6.1: Probability of Meeting Performance Tolerance

\begin{tabular}{lllllllll}
\hline & \multicolumn{7}{c}{ Number of Initial Arrangements } \\
\cline { 2 - 8 } Tolerance & 1 & 2 & 3 & 4 & 5 & 6 & 7 \\
\hline \hline $0 \%$ & 0.40 & 0.64 & 0.79 & 0.87 & 0.92 & 0.95 & 0.97 \\
$5 \%$ & 0.67 & 0.84 & 0.91 & 0.94 & 0.96 & 0.97 & 0.98 \\
$15 \%$ & 0.95 & 0.99 & 1.00 & 1.00 & 1.00 & 1.00 & 1.00 \\
\hline
\end{tabular}

\subsection{Case Studies}

To evaluate the performance of the new algorithms a series of case studies are performed. First, Section 6.4.1 examines the prevalence of local minima within the design space. Next, in Section 6.4.2, test cases examine the performance of stochastic descent as the number of neighbors checked varies. Finally, Section 6.4.3 examines test cases to compare the performance of stochastic and steepest descent with am exhaustive search algorithm. In all test cases the results use converter data from the TI WEBENCH design tool [29].

\subsubsection{Local Minima}

To examine the prevalence of local minima the test cases in [10] and [49] are examined. For each test case the problem is solved using steepest decent starting from every feasible arrangement. A histogram of the local minima's score from each starting arrangement can be seen in Fig. 6.4. These results show that local minima are rare and in $81 \%$ of the cases' local minima are within $10 \%$ of the optimal.

This test case also provides insight into the selection of the number of initial arrangements. As shown in Table 6.1 to ensure a $90 \%$ probability that the global optimal solution is found five initial arrangements must be used. However, if a solution within $5 \%$ or $15 \%$ of the optimal is acceptable only three or one initial arrangements are required respectively. 


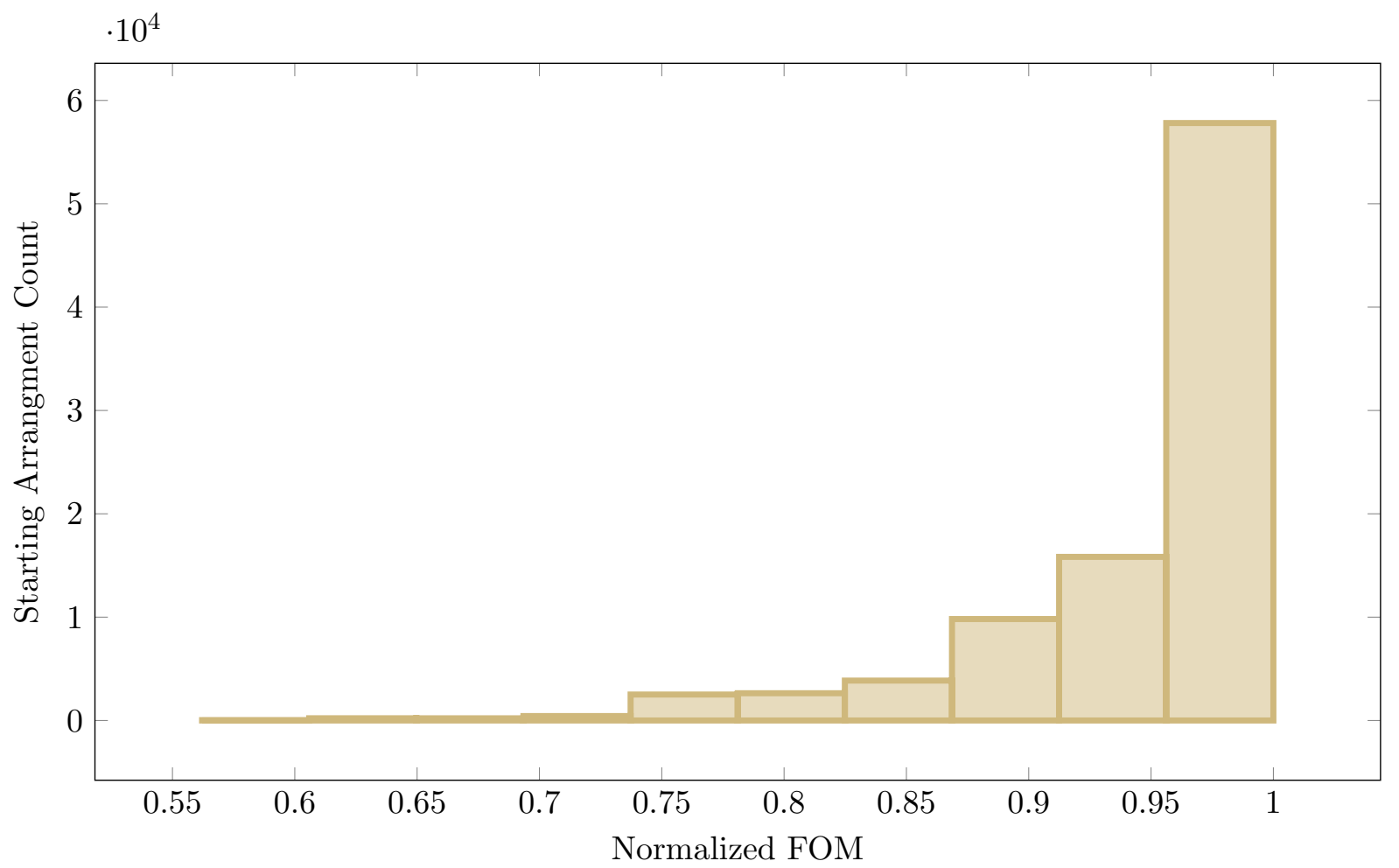

Figure 6.4: Histogram of normalized FOM for the optimal system found from each possible starting arrangement in six test cases. In $81 \%$ of cases the optimal value is within $10 \%$ of the global optimum demonstrating that there are few local minima far from the global optimum.

\subsubsection{Stochastic Descent Neighbors}

To examine how the number of neighbors evaluated affects the performance of the stochastic descent technique six test cases are solved using stochastic descent with varying numbers of neighbors evaluated. Fig. 6.5 shows the normalized system FOM and run time as functions of the percent of neighbors evaluated. Each line is a linear line of best fit for the combined test cases' results. These results show that stochastic descent can find the true optimal by evaluating a single neighbor while reducing the computational time by roughly $60 \%$. This verifies the analysis in Sections 6.2 .1 and 6.3.1 where it was shown that stochastic descent reduced the number of arrangements that must be evaluated at 15 outputs by approximately $50 \%$. 


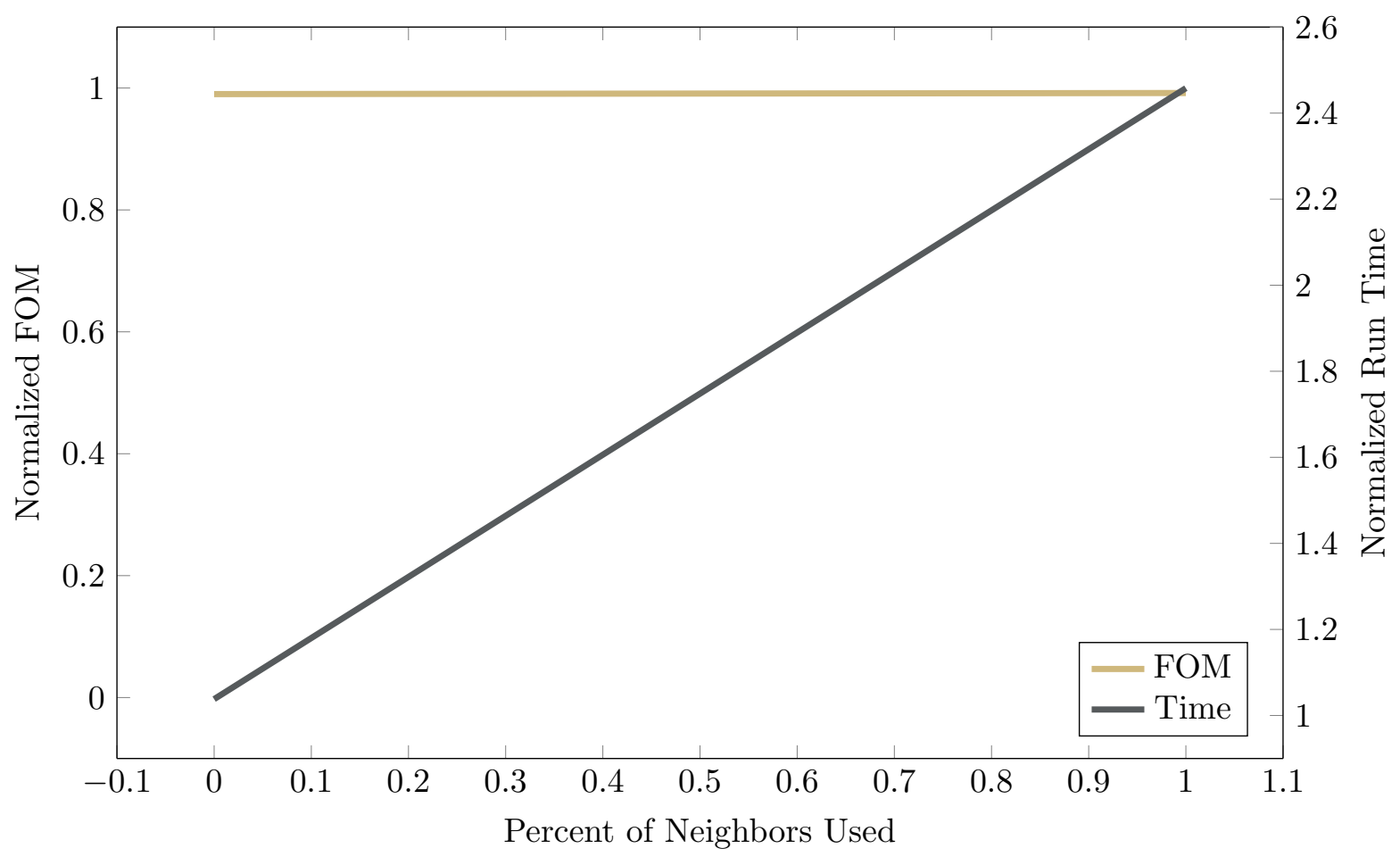

Figure 6.5: Normalized stochastic descent optimal FOM and run time as functions of the percent of neighbors used. By descending before evaluating all neighbors the run time can be reduced considerably without any reduction in the final optimal FOM. Lines are best fit lines from the results of six test cases.

\subsubsection{Performance Comparison}

To evaluate the performance of the new optimization algorithm a series of case studies are performed and a comparison can be seen in Fig. 6.6. For each case the input is a $48 \mathrm{~V}$ bus, and the optimal is found with steepest descent, stochastic descent, exhaustive search, and a heuristically modified exhaustive search. In all cases the optimization goal is system FOM. The "small" system has 7 outputs: 3 high current, low voltage outputs, 2 analog voltage rails, a digital voltage rail, and a high voltage node. The "medium" system with 8 outputs adds another low voltage output for an additional processor. In the "large" system a processor [42] and FPGA [43] must be powered in addition to the analog, digital, and high-voltage loads. As discussed in Chapter 5 the exhaustivesearch algorithm enumerates all possible arrangements, selects converters to realize each, and selects the best architecture. The heuristics group together similar nodes or eliminate arrangements with 


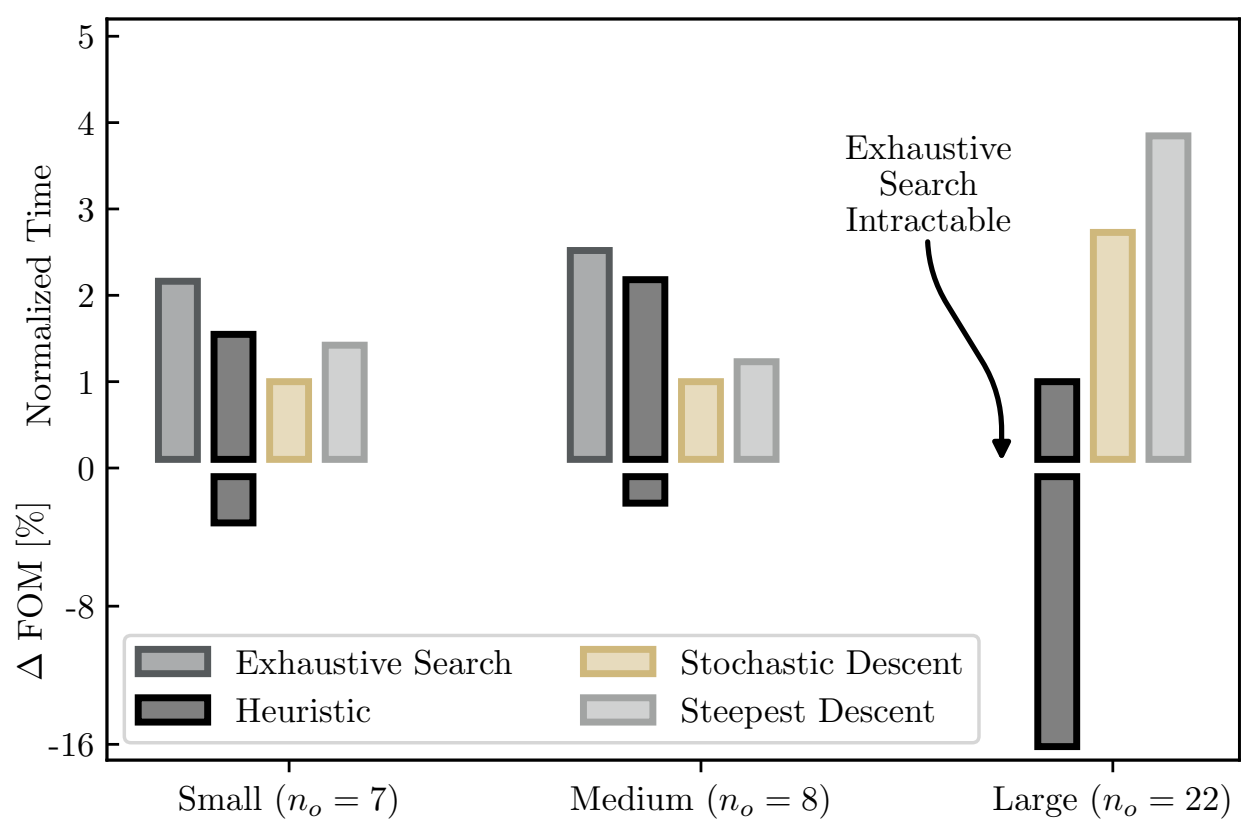

Figure 6.6: Comparison between exhaustive search, steepest descent, stochastic descent, and simulated annealing for three systems of varying size. Results show that stochastic descent finds a comparable or better solution for all test cases; however, it has $10 \%$ to $40 \%$ longer solve times compared to simulated annealing. The small system contains 7 nodes, the medium contains 8 , and the large contains 22. The large system could not be solved using an exhaustive search. Results are normalized by the best result for each test case. A missing bar for FOM indicates that the technique found the optimal solution. Each bar represents the average of three runs.

large conversion ratios. Intermediate bus nodes may also be inserted in cases when large conversion ratios are required. The solution run times can be seen in Table 7.2. For each test case the algorithm is run three times and the average run time is reported. Times were recorded using a desktop computer with an i7-7700 CPU running at 3.6 GHz.

\subsubsection{Small Test Case}

The small test case contains seven outputs as shown in Table 6.3 and is supplied by a $48 \mathrm{~V}$ input. The optimal solution as found by stochastic descent and exhaustive search is shown in Fig. 6.8 where the graph edges are scaled proportional to the logarithm of the power processed. In the small case stochastic descent is the only efficient technique which finds the same optimal as exhaustive search every time and it does so in $54 \%$ less time than the exhaustive search. 
Table 6.2: Average Solve Time

\begin{tabular}{|c|c|c|c|c|}
\hline Case & Exthaust & ste $e^{S e a r c h t}$ & $\begin{array}{l}\text { Descent } \\
\text { stee } \\
e^{e s t}\end{array}$ & Descent \\
\hline Small & $5: 41: 30$ & $2: 37: 54$ & $3: 44: 36$ & $4: 04: 25$ \\
\hline Medium & $8: 15: 37$ & $3: 16: 45$ & $4: 02: 13$ & 7:09:10 \\
\hline Large & & 29:13:03 & $41: 11: 25$ & $10: 42: 31$ \\
\hline All Converters - $5 \mathrm{~W}$ & $3: 36: 18$ & $2: 30: 14$ & $2: 37: 58$ & $3: 03: 44$ \\
\hline All Converters - $10 \mathrm{~W}$ & $3: 30: 30$ & $1: 55: 54$ & $2: 17: 27$ & $2: 31: 07$ \\
\hline All Converters - $15 \mathrm{~W}$ & $3: 04: 53$ & $1: 46: 03$ & $2: 15: 05$ & $3: 10: 26$ \\
\hline All Converters - $25 \mathrm{~W}$ & $4: 07: 05$ & $2: 46: 08$ & $3: 10: 50$ & $3: 16: 11$ \\
\hline All Converters - $35 \mathrm{~W}$ & $4: 18: 45$ & $2: 47: 49$ & $3: 14: 42$ & $3: 07: 29$ \\
\hline All Converters - $50 \mathrm{~W}$ & $5: 34: 21$ & $2: 45: 37$ & $3: 29: 30$ & $5: 35: 26$ \\
\hline Low Voltages - $5 \mathrm{~W}$, Others - $5 \mathrm{~W}$ & $3: 36: 25$ & $2: 37: 12$ & $2: 36: 58$ & $3: 03: 44$ \\
\hline Low Voltages - $10 \mathrm{~W}$, Others - $5 \mathrm{~W}$ & $3: 53: 55$ & $2: 19: 24$ & $2: 26: 26$ & $3: 05: 59$ \\
\hline Low Voltages - $15 \mathrm{~W}$, Others $-5 \mathrm{~W}$ & $3: 23: 08$ & $2: 04: 25$ & $2: 24: 52$ & $3: 32: 32$ \\
\hline Low Voltages - $25 \mathrm{~W}$, Others - $5 \mathrm{~W}$ & 4:08:39 & $2: 53: 43$ & $3: 00: 46$ & $3: 04: 34$ \\
\hline Low Voltages - $35 \mathrm{~W}$, Others - $5 \mathrm{~W}$ & $4: 13: 49$ & $2: 54: 15$ & $3: 26: 58$ & $3: 20: 26$ \\
\hline Low Voltages - $50 \mathrm{~W}$, Others - $5 \mathrm{~W}$ & $5: 26: 50$ & $2: 33: 53$ & $3: 57: 58$ & $5: 21: 51$ \\
\hline
\end{tabular}




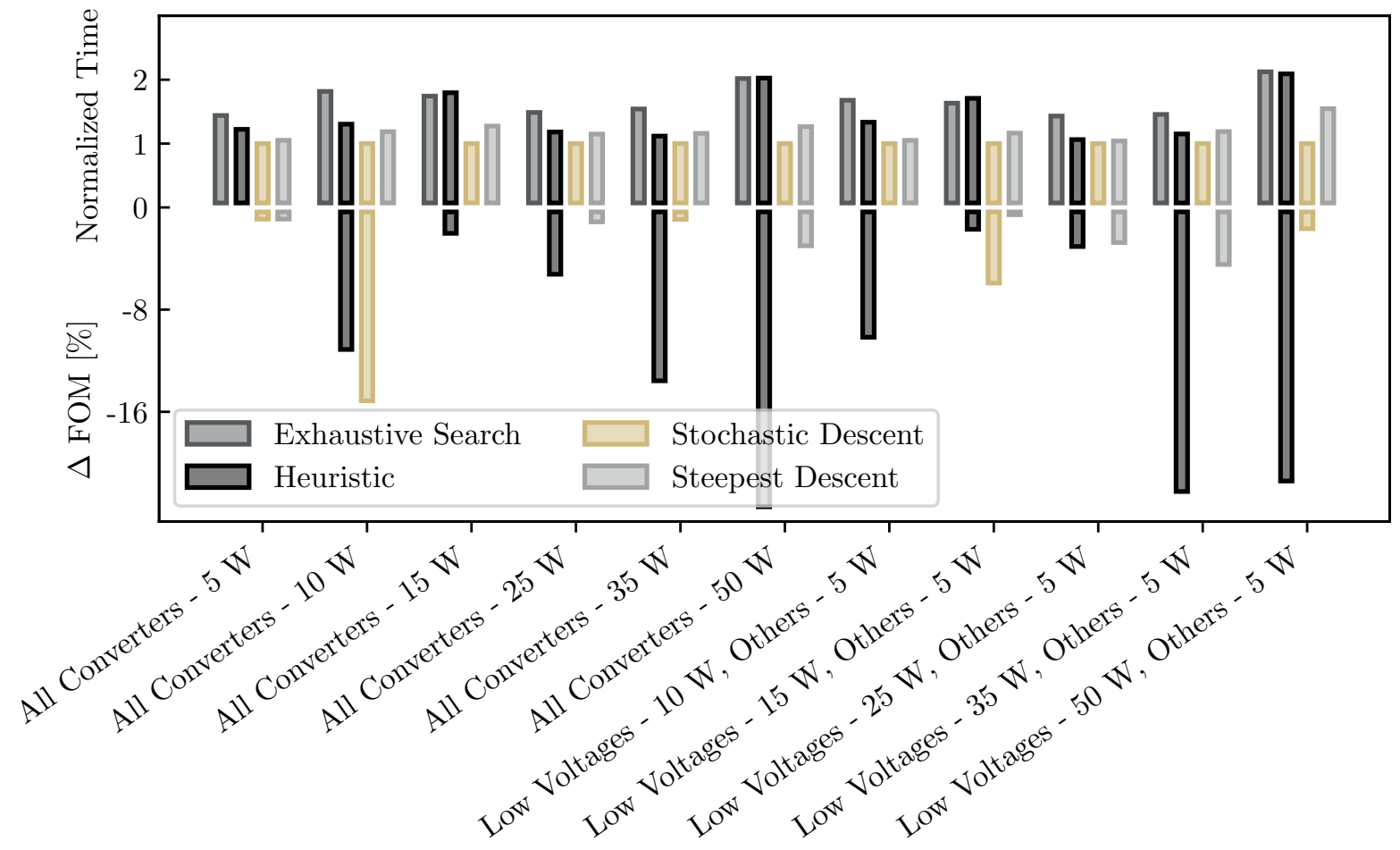

Figure 6.7: Comparison between considered techniques for test cases described in [10] each with seven outputs.

Table 6.3: Outputs in the small system

\begin{tabular}{ll}
\hline Volts & Amps \\
\hline \hline 24 & 1 \\
12 & 1 \\
5 & 5 \\
1 & 10 \\
1.1 & 10 \\
1.3 & 10 \\
3.3 & 5 \\
\hline
\end{tabular}




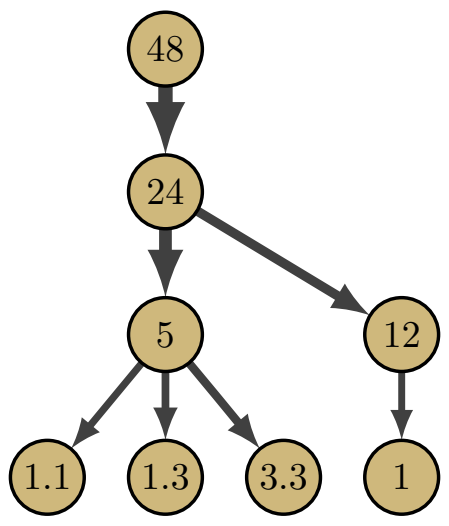

Figure 6.8: Optimal arrangement for the small test case. Edge widths are proportional to the logarithm of the power processed by the converter it represents.

\subsubsection{Medium Test Case}

The medium test case contains 8 output nodes, as shown in Table 6.4 and is supplied by a $48 \mathrm{~V}$ input. The optimal solution as found by the exhaustive-search and steepest descent algorithms is shown in Fig. 6.9. For the medium case steepest descent is the only efficient technique to find the same optimal as the exhaustive search which it does in $51 \%$ less time than the exhaustive search. Stochastic descent and simulated annealing both find solutions in slightly less time than steepest descent, but are slightly less consistent in finding the global optimal system.

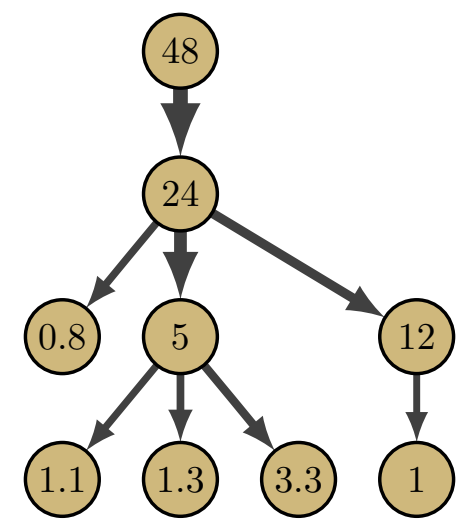

Figure 6.9: Optimal arrangement for the medium test case. Edge widths are proportional to the logarithm of the power processed by the converter it represents. 
Table 6.4: Outputs in the medium system

\begin{tabular}{ll}
\hline Volts & Amps \\
\hline \hline 24 & 1 \\
12 & 1 \\
5 & 5 \\
1 & 10 \\
1.1 & 10 \\
1.3 & 10 \\
3.3 & 5 \\
0.8 & 15 \\
\hline
\end{tabular}

\subsubsection{Large Test Case}

The large test case contains 22 output nodes as shown in Table 6.5 and is also supplied by a $48 \mathrm{~V}$ input. The optimal solution as found by stochastic descent is shown in Fig. 6.10. The progress of both descent algorithms for the large case is shown in Fig. 6.11. The steepest descent clearly descends faster from a descent step point of view, but it's important to note that stochastic must evaluate fewer arrangements for each step, so it is still faster despite requiring more steps to reach the optimal.

Similar to the conclusions in [41], [49] there are several properties of the optimal system of interest. The first is that the optimal solution is very likely to be at a depth of two or three. While the optimal solution in the large problem does have a depth of four all of the nodes deeper than the third level are small and could likely be moved up a level with minimal impact on the system performance. Second, it can again be seen that "super nodes" as described in Section 2.4.1 are likely to produce suboptimal systems. There is more than voltage that determines which nodes are likely to share a parent. From these results it appears that a heuristic which also takes into account the power of a node's load would be a more effective predictor of shared parents. The highest power nodes are likely to be moved away from where voltage only heuristics would place them, whereas low power nodes are likely to be grouped with one another. This may not be true in all systems though, in this test case roughly half of the outputs have high power loads and the other half are low power. In systems where all nodes have similar power requirements voltage based heuristics 


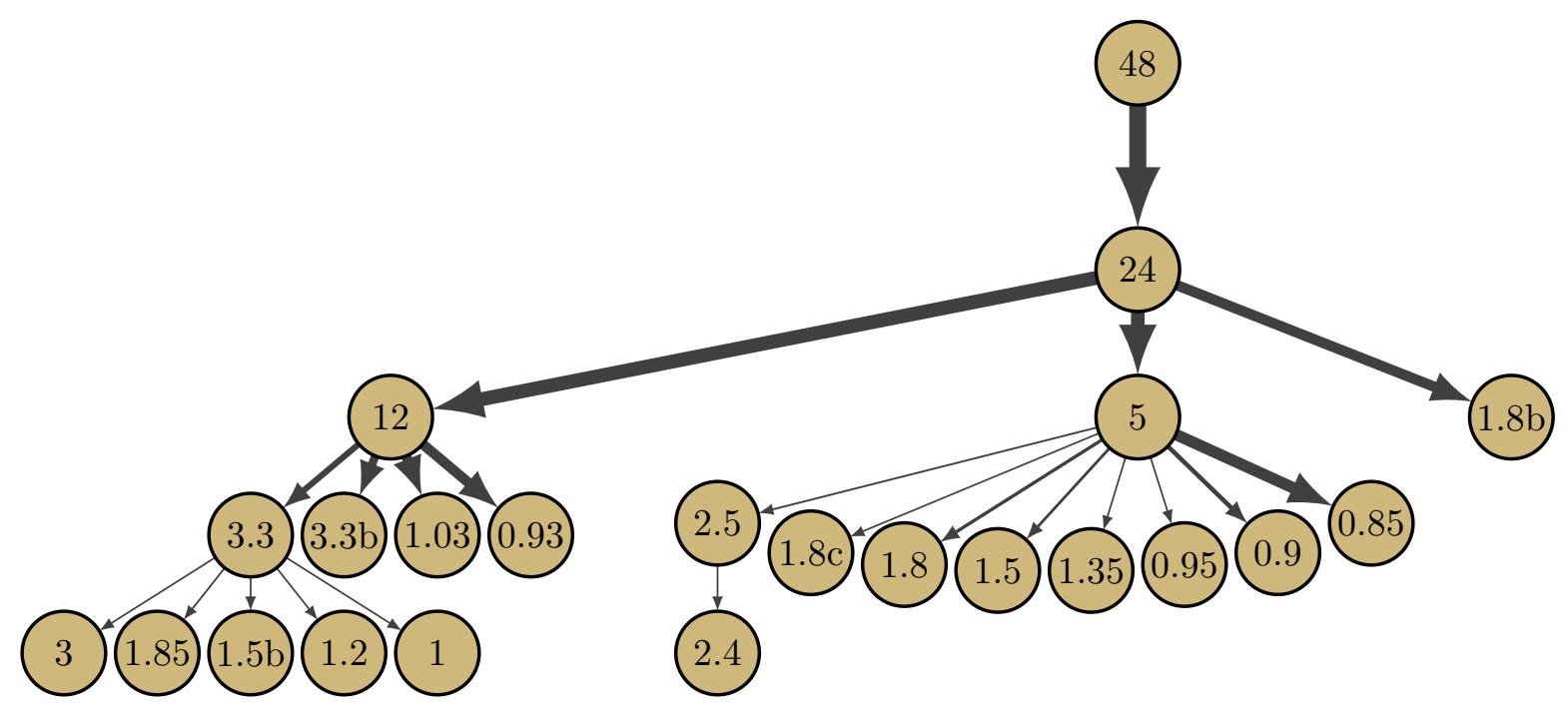

Figure 6.10: Optimal arrangement for the large test case found with stochastic descent. Edge widths are proportional to the logarithm of the power processed by the converter it represents.
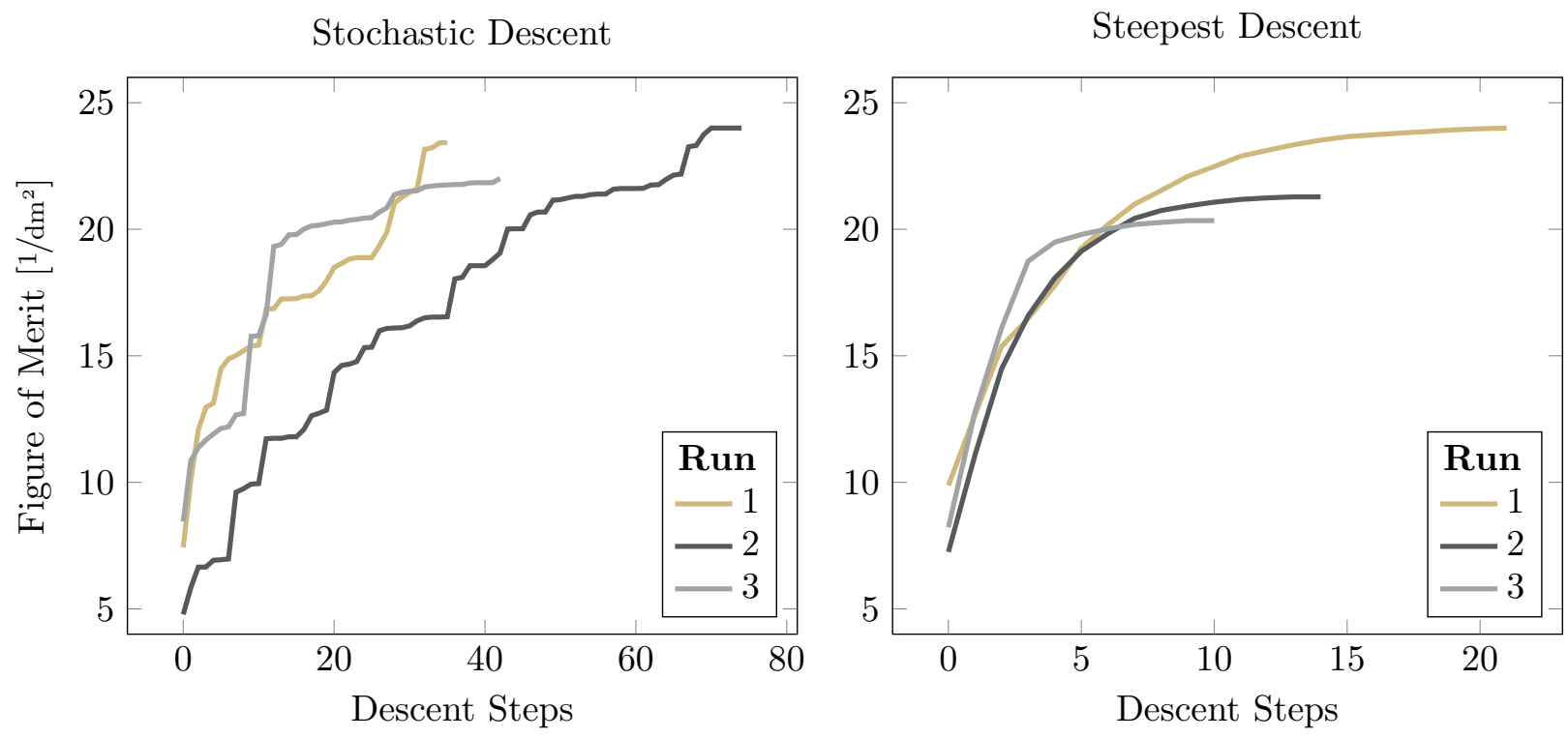

Figure 6.11: Comparison of the descent progress while solving the large test case for stochastic descent (left) and steepest descent (right). As steepest descent evaluates all neighbors and descends to the best it initially descends very rapidly to architectures with improved FOMs. Stochastic descent, on the other hand, descends as soon as it finds an improved solution so its steps are much smaller and it must take more before it reaches a minima. However, because it does not need to evaluate all neighbors before descending it is still faster than steepest descent. Each line represents a different starting arrangement. 
Table 6.5: Outputs in the large system

\begin{tabular}{ll}
\hline Volts & Amps \\
\hline \hline 0.85 & 55.4 \\
0.9 & 5.1 \\
0.93 & 32.0 \\
0.95 & 0.25 \\
1 & 0.75 \\
1.03 & 27.9 \\
1.2 & 0.75 \\
1.35 & 0.75 \\
\hline
\end{tabular}

\begin{tabular}{ll}
\hline Volts & Amps \\
\hline \hline 1.5 & 1.7 \\
$1.5 \mathrm{~b}$ & 0.75 \\
1.8 & 1.7 \\
$1.8 \mathrm{~b}$ & 18.2 \\
$1.8 \mathrm{c}$ & 0.75 \\
1.85 & 0.98 \\
2.4 & 0.001 \\
2.5 & 0.75 \\
\hline
\end{tabular}

\begin{tabular}{ll}
\hline Volts & Amps \\
\hline \hline 3 & 0.02 \\
3.3 & 0.75 \\
$3.3 \mathrm{~b}$ & 5.0 \\
5 & 5.0 \\
12 & 1.0 \\
24 & 2.0 \\
& \\
\hline
\end{tabular}

will likely be better predictors.

\subsection{Conclusions}

By describing a meaningful design space with connections to similar arrangements optimization of dc distribution architectures can be effectively solved using descent based methods. In this thesis two descent based methods, steepest descent and stochastic descent, are described and implemented. Detailed analysis of the design space and the way the presented techniques travel through it show how descent based techniques can reduce the number of evaluated arrangements from the trillions in an exhaustive search approach to thousands or hundreds. Test cases are presented which compare the performance of descent based methods to an exhaustive search and also to recently described simulated annealing based solvers. Results from these test cases show that descent based methods can find the same or better solutions than simulated annealing without requiring any performance modifying hyperparameters. Despite slightly longer solve times than simulated annealing, the simplicity and ease of implementation of descent based methods make them a good choice when optimizing de distribution architectures. 


\section{Chapter 7}

\section{Simulated Annealing}

Simulated Annealing is a probabilistic optimization procedure first developed in the 1980s for a variety of non-convex optimization problems where the cost function may have several local minima [50], [51]; it is particularly utilized when the optimization problem involves discrete variables, including problems associated with integrated circuit design and layout and the traveling salesman problem [50]-[53]. The theoretical convergence of simulated annealing to a globally optimal solution is provided in, e.g., [54]. This chapter is structured as follows: first a brief review of simulated annealing is given in Section 7.1, then the implementation details for a power electronics system are described in Section 7.2, and finally a set of case studies are examined in Section 7.3.

\subsection{Simulated Annealing Review}

The main ingredients of simulated annealing are a finite set, a real-valued cost function to be minimized (with the set of globally optimal solution being a proper subset), and a nonincreasing function called the "temperture". As the name suggests, its operation is designed to mimic the process of cooling a metal during annealing. The optimization procedure starts with a high temperature where perturbations that increase the cost function are accepted with a high probability. This allows the process to escape local minima as it moves through the design space. As the procedure continues the temperature is gradually lowered and the probability of moving to less optimal solutions is reduced. Finally the temperature of the system is reduced low enough that new changes are infrequently accepted and the system is considered "frozen."

General operation of simulated annealing can be seen in Fig. 7.1. To begin, a starting 


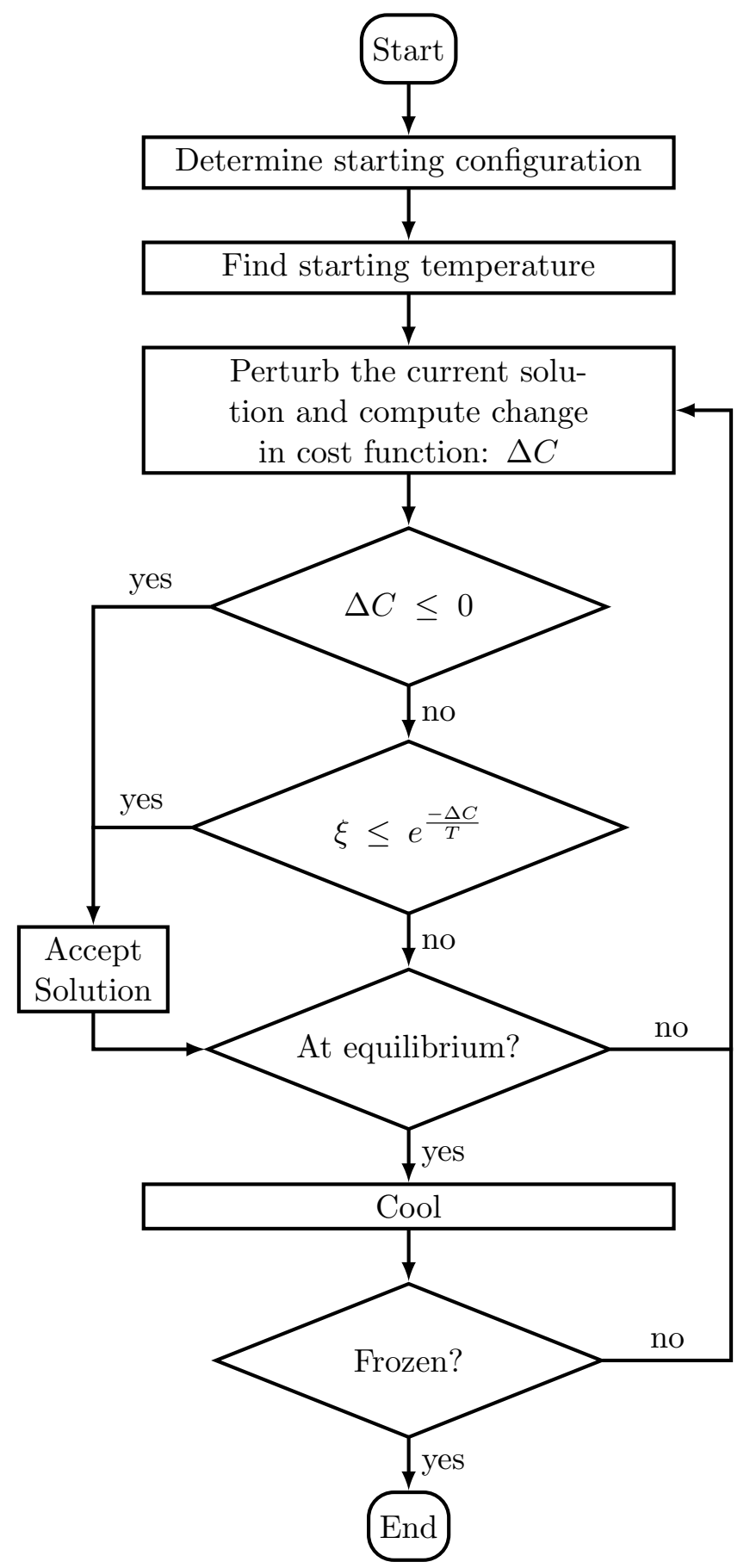

Figure 7.1: Flowchart for operation of a generic simulated annealing algorithm. $\xi$ is a random variable with a uniform distribution between zero and one. 
configuration must be chosen. This can be done with a heuristic, as is commonly done with the traveling salesman problem (TSP), or it may be chosen randomly. Then a starting temperature must be chosen to meet a tradeoff between solution time and performance. Once these choices have been made the algorithm enters its main loop. The first step in the loop is to perturb the current solution to generate a new solution that is only different from the previous one by a small amount. For example, in the TSP a common choice is to swap the travel order of two adjacent nodes. Next, the cost of the perturbed solution is compared to the current solution. If the perturbed solution is an improvement it is accepted and the current solution is updated to the perturbed solution. If it is not an improvement the solution will be accepted with probability

$$
e^{\frac{-\Delta C}{T}}
$$

where $\Delta C$ is the change in cost between the current solution and the perturbed solution and $T$ is the temperature of the system. Once a perturbation has been accepted or rejected the system checks if it is at "equilibrium." If it is not at equilibrium the algorithm continues perturbing the current solution until it reaches equilibrium at which point the system will cool. Once it has cooled the system checks if it is frozen and otherwise continues with another cooling cycle. Once the system is frozen, the optimal solution is the best solution encountered at any time during the optimization procedure, which is ideally the last solution.

\subsection{Optimization Algorithm Using Simulated Annealing}

While the top level view of simulated annealing is straightforward there are non-trivial application specific choices and implementation details. This section describes the optimization algorithm implementation for DC distribution architectures with focus on the components that require special attention due to the power electronics nature of the system.

\subsubsection{Input System Structure}

As in Chapter 2 the input system (i.e. the system to be optimized) is represented as a directed graph. This "characteristic graph" contains a node for every output required in the system and 
a node for the system input as shown in Fig. 2.2. In the characteristic graph there are directed edges from nodes to other nodes where a converter may be placed. An assumption is made that each output below the input will only have edges into it from either the input or from another node below the input that represents a larger voltage and vice versa for outputs above the input. This decision decouples the problem into two separate optimization problems, a "boosting" system with output voltages above the input voltage, and a "bucking" system with voltages below the input.

\subsubsection{Initial Configuration}

To initialize the optimization procedure an arrangement is chosen randomly and converters are selected according to the optimization goal. A random arrangement may be generated by selecting an input edge from the characteristic graph at random for each node in the system. As the graph is directed and there are no edges moving back up the graph (in the bucking case) the characteristic graph is acyclic and this process is guaranteed to generate an arborescence. It is, however, possible to generate an infeasible initial configuration if there are no converters available for one of the edges or if the arrangement cannot meet startup order requirements. Multiple attempts may therefore be required to arrive at the initial configuration.

\subsubsection{Choosing the Initial Temperature}

Setting the initial temperature is done with a procedure described in [51]. At the beginning of the simulated annealing process it is generally desired to have a relatively high acceptance rate when a perturbation does not improve the system. This is to eliminate any dependence in the final outcome on the initial configuration and also to ensure that it is easy to escape local minima. In order to achieve a target initial acceptance, $\chi_{0}$, the initial configuration is perturbed $n_{\text {init }}$ times and when the solution is not improved the change in cost is recorded. The acceptance expression can then be solved for $T$ with the average cost increase as $\Delta C$ and $\chi_{0}$ as the acceptance probability

$$
T_{0}=\frac{\Delta C^{+}}{\ln \left(\chi_{0}^{-1}\right)}
$$




\subsubsection{Perturbing the System}

There are two types of system perturbations: the first is to change the input for a node and the second is to insert or remove an Intermediate Bus Node (IBN) in the system. IBNs are described in [25] and their usage in DC distribution architecture design is shown in Section 2.4. In certain systems they may improve the performance of high conversion ratio stages that may otherwise be inefficient or infeasible. To change the input for a node its current input edge is removed and a new edge is selected randomly from the list of edges into it in the characteristic graph as well as the edges from any IBNs present in the current solution that represent a larger voltage. Additionally, any IBNs left at the tip of a branch after the perturbation should be removed. Adding or removing an IBN is more complicated as there may be multiple IBNs chained together. When adding or removing an IBN from a chain that contains IBNs the other IBNs are dispersed such that each converter in the chain has an identical conversion ratio.

With these two perturbation types perturbing a solution is done as follows: first a node from the current solution is selected at random. If it is an output node an intermediate bus node is inserted if $\xi \leq \zeta_{I B N}$ otherwise the input edge is changed. If the selected node is an IBN it is split if $\xi \leq \zeta_{\text {split }}$ otherwise it is removed. As when initializing, any infeasible solution is rejected.

\subsubsection{Checking Equilibrium}

Equilibrium is achieved if the number of accepted solutions since the last cooling cycle is equal to $l_{\text {chain }}$. When the system has cooled to an almost frozen state it is possible that there are no perturbations that would be accepted, so the system should also be considered at equilibrium if system would freeze after cooling.

\subsubsection{Cooling}

The system is cooled to a fraction of its current temperature each cooling cycle as

$$
T_{k+1}=\alpha_{c} T_{k}
$$

where $\alpha_{c} \in(0,1)$ is a given constant. 


\subsubsection{Freezing}

There are two conditions that the system may meet to be considered frozen. The first is if the acceptance ratio during the previous cooling cycle fell below $\chi_{\min }$. The second is when the solution has changed by less than $\beta_{\text {stall }}$ percent in the last $n_{\text {stall }}$ cooling cycles and the acceptance rate is below $\chi_{\max }$. As mentioned in Section 7.2.5 the system will also be considered at equilibrium if it would be frozen after cooling. However, at the beginning of each cooling cycle the acceptance ratio may be artificially low if the first few perturbations are rejected. Therefore, the freezing logic must ensure that during this check the system is not considered frozen if there are only a handful of rejections. To accomplish this the algorithm only considers the acceptance rate if there have been at least

$$
N_{r}=\left\lceil\sqrt{l_{\text {chain }}}\right\rceil\left(\frac{1}{\chi_{\min }}-1\right)
$$

rejections in the current cooling cycle. This prevents the system from freezing before the first $\left\lceil\sqrt{l_{\text {chain }}}\right\rceil$ accepted solutions if there are not enough rejections to reduce the acceptance ratio below $\chi_{\min }$ given as many accepted solutions.

\subsubsection{System Evaluation}

During the optimization process there are multiple points where the solution must be evaluated to assign a score or cost. In this chapter this is accomplished as in Chapter 4. The solutions generated by simulated annealing are arrangements without converters and to assign a cost they are first populated with converters chosen to meet the optimization goal of the particular application. This can either be maximum efficiency, smallest area, lowest cost, or some other custom FOM. Once converters are selected the system cost can be computed and used in the simulated annealing process. Care is required during this step to ensure that the optimization procedure moves in the correct direction. Depending on the specific cost used it may be desirable to maximize the cost instead of minimize it as is the case when the cost is FOM. For a given cost $\Delta C$ must be computed such that it is negative when the perturbed system represents an improvement and positive otherwise. 
Table 7.1: Simulated Annealing Hyperparameters

\begin{tabular}{llll}
\hline Parameter & Symbol & Normal Value & Slow Value \\
\hline \hline Initial Perturbations & $n_{\text {init }}$ & $n_{o}$ & $n_{o}$ \\
Initial Acceptance & $\chi_{0}$ & $5 \%$ & $70 \%$ \\
Chain Length & $l_{\text {chain }}$ & $\left\lceil\sqrt{n_{o}}\right\rceil$ & $n_{o}$ \\
Cooling Fraction & $\alpha_{c}$ & $70 \%$ & $95 \%$ \\
IBN Probability & $\zeta_{\text {IBN }}$ & $0 \%$ & $0 \%$ \\
IBN Split & $\zeta_{\text {split }}$ & $50 \%$ & $50 \%$ \\
Max Final Acceptance & $\chi_{\text {max }}$ & $15 \%$ & $15 \%$ \\
Min Final Acceptance & $\chi_{\text {min }}$ & $0.5 \%$ & $4 \%$ \\
Stall Chains & $n_{\text {stall }}$ & 10 & 5 \\
Stall Tolerance & $\beta_{\text {stall }}$ & $0.5 \%$ & $0.5 \%$ \\
\hline
\end{tabular}

\subsubsection{Parameter Tuning}

Careful selection of parameters is critical to the performance of simulated annealing and is itself a multi-objective optimization problem balancing solution performance and run time. In this work two sets of parameters are considered and the values for each are shown in Table 7.1. The "slow" version of simulated annealing is based on the typical values used in simulated annealing literature to solve other problems. The initial acceptance rate is high, cooling is applied slowly, and the conditions for freezing are focused on the minimum acceptance ratio. Test cases (see Section 7.3) show that this approach is highly effective at finding the optimal solution; however, there is significant oscillation in the progress and the convergence is relatively slow. In contrast, the fast version has a low initial acceptance rate, cools considerably quicker, and its freezing condition is focused on the number of stall chains, only freezing based on acceptance rate when almost all possible perturbations have been rejected. This technique is considerably faster than the slow version and is still effective at finding the optimal or near-optimal solution in many of the considered test cases. 
Table 7.2: Average Solve Time

\begin{tabular}{|c|c|c|c|c|c|c|}
\hline Case & $510 \times 5$ & singuted & An neatis & je Search & D $^{e^{s} e^{e n t}}$ & Descent \\
\hline Small & $4: 11: 13$ & $2: 03: 27$ & $5: 41: 30$ & $2: 37: 54$ & $3: 44: 36$ & 4:04:25 \\
\hline Medium & $5: 26: 18$ & $2: 59: 32$ & $8: 15: 37$ & $3: 16: 45$ & 4:02:13 & $7: 09: 10$ \\
\hline Large & $51: 48: 36$ & $21: 14: 06$ & & $29: 13: 03$ & $41: 11: 25$ & $10: 42: 31$ \\
\hline All Converters $-5 \mathrm{~W}$ & $3: 14: 12$ & $1: 44: 19$ & $3: 36: 18$ & $2: 30: 14$ & $2: 37: 58$ & 3:03:44 \\
\hline All Converters - $10 \mathrm{~W}$ & $2: 16: 51$ & $1: 43: 41$ & $3: 30: 30$ & $1: 55: 54$ & $2: 17: 27$ & $2: 31: 07$ \\
\hline All Converters - $15 \mathrm{~W}$ & $2: 35: 14$ & $1: 50: 53$ & $3: 04: 53$ & $1: 46: 03$ & $2: 15: 05$ & $3: 10: 26$ \\
\hline All Converters - $25 \mathrm{~W}$ & $3: 33: 44$ & $2: 11: 44$ & 4:07:05 & $2: 46: 08$ & $3: 10: 50$ & $3: 16: 11$ \\
\hline All Converters - $35 \mathrm{~W}$ & $3: 56: 51$ & $2: 37: 49$ & $4: 18: 45$ & $2: 47: 49$ & $3: 14: 42$ & $3: 07: 29$ \\
\hline All Converters - $50 \mathrm{~W}$ & $3: 32: 32$ & $2: 27: 47$ & $5: 34: 21$ & $2: 45: 37$ & $3: 29: 30$ & $5: 35: 26$ \\
\hline Low Voltages - $5 \mathrm{~W}$ & $3: 08: 29$ & $1: 51: 50$ & $3: 36: 25$ & $2: 37: 12$ & $2: 36: 58$ & 3:03:44 \\
\hline Low Voltages - $10 \mathrm{~W}$ & $2: 52: 57$ & $2: 04: 16$ & $3: 53: 55$ & $2: 19: 24$ & $2: 26: 26$ & $3: 05: 59$ \\
\hline Low Voltages - $15 \mathrm{~W}$ & $3: 01: 34$ & $1: 42: 36$ & $3: 23: 08$ & $2: 04: 25$ & $2: 24: 52$ & $3: 32: 32$ \\
\hline Low Voltages $-25 \mathrm{~W}$ & $3: 43: 16$ & $2: 14: 28$ & 4:08:39 & $2: 53: 43$ & $3: 00: 46$ & $3: 04: 34$ \\
\hline Low Voltages $-35 \mathrm{~W}$ & $3: 43: 28$ & $2: 02: 46$ & $4: 13: 49$ & $2: 54: 15$ & $3: 26: 58$ & $3: 20: 26$ \\
\hline Low Voltages - $50 \mathrm{~W}$ & $4: 10: 20$ & $2: 21: 54$ & $5: 26: 50$ & $2: 33: 53$ & $3: 57: 58$ & $5: 21: 51$ \\
\hline
\end{tabular}

\subsection{Case Studies}

To evaluate the performance of the simulated annealing optimization algorithm a series of case studies are performed and a comparison can be seen in Fig. 7.2. For each case a series of outputs are specified and the optimal solution is found using simulated annealing, using the exhaustive-search

algorithm in Chapter 5, and using the stochastic descent algorithm described in Chapter 6. The cases are identical to those examined in Section 6.4.3.

One important factor in any test case is the source of converter data used to realize the architecture. As discussed in [41] this data may come from many sources. In this work TI's 


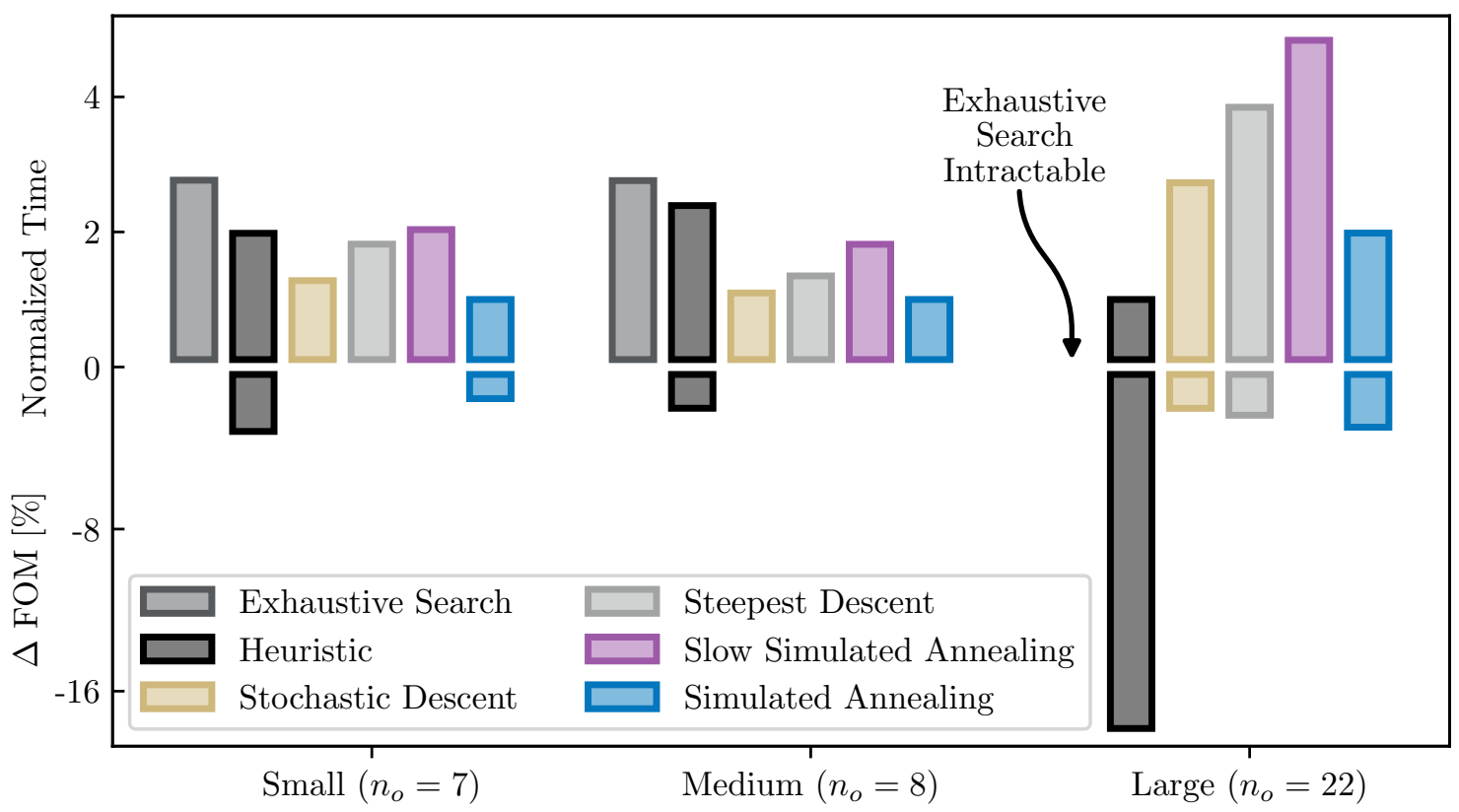

Figure 7.2: Comparison between normal and slow simulated annealing, exhaustive search, and exhaustive search with heuristically modified design space [10] for three systems of varying size. Results show that simulated annealing finds a comparable solution in $30 \%$ to $50 \%$ of the time for each test case. An alternate simulated annealing implementation finds the optimal solution and is still more computationally efficient than exhaustive search in all cases. Heuristics are slower and perform worse in the small and medium cases and perform considerably worse in the large case. The small system contains 7 nodes, the medium contains 8, and the large contains 22 . For the heuristic method the small and medium systems allowed a single intermediate bus voltage and the number of independent nodes was reduced by 1 . In the large system the number of independent nodes was limited to 9 . In all three cases the maximum conversion ratio was set to 10 . The large system could not be solved using an exhaustive search without heuristics. Results are normalized by the best result for each test case. A missing bar for FOM indicates that the technique found the optimal solution. Each bar represents the average of three runs. 


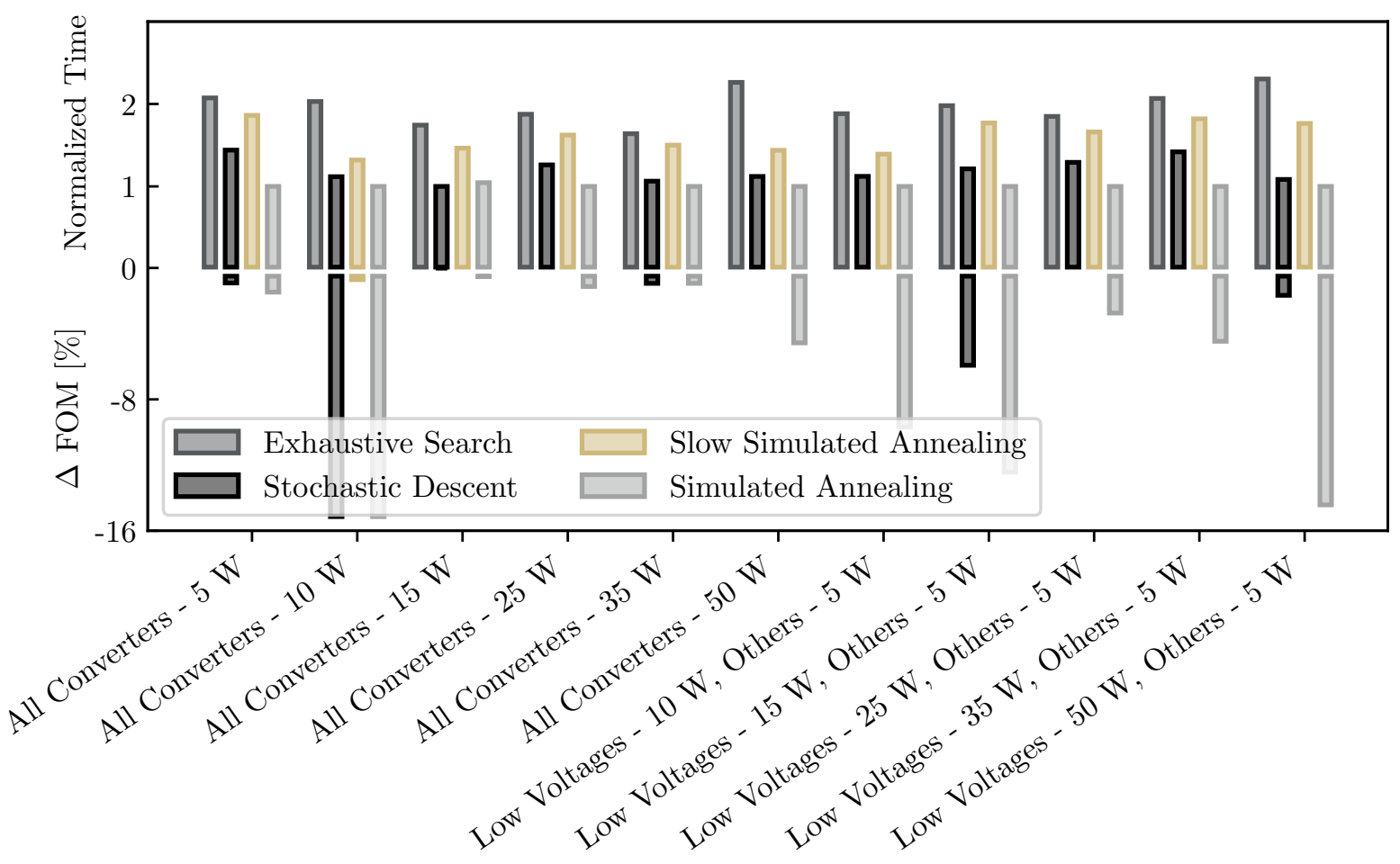

Figure 7.3: Comparison between considered techniques for test cases described in [10] each with seven outputs.

WEBENCH [29] software is used; this source provides realistic, high-quality converter designs, but must be accessed through a website, so database access is relatively slow. However, there is nothing about the proposed algorithm that limits it to this particular converter database, or prevents it from utilizing more sophisticated techniques to chose converters such as designing custom converters using an automated design procedure.

\subsubsection{Small Test Case}

The small test case contains seven outputs as shown in Table 6.3 and is supplied by a $48 \mathrm{~V}$ input. The optimal solution is shown in Fig. 6.8 where the graph edges are scaled proportional to the logarithm of the power. For this case simulated annealing is $63.9 \%$ faster and produces a result with a figure of merit within $2 \%$ of the true optimal solution obtained by the exhaustivesearch method. The slow simulated annealing approach finds the true optimal solution in less time 


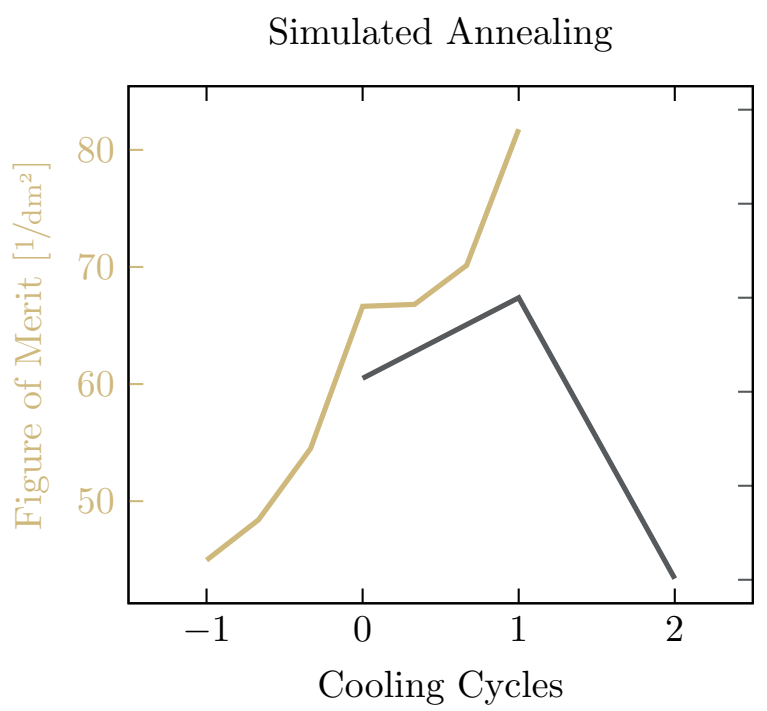

Slow Simulated Annealing

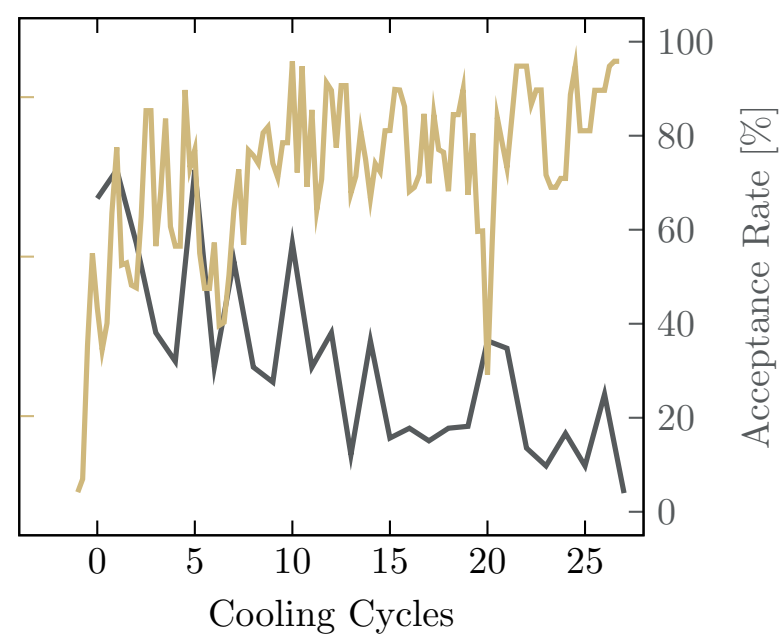

Figure 7.4: Comparison of the simulated annealing progress while solving the small test case for the normal (left) and slow (right) versions of the algorithm.

than the exhaustive search. The solution obtained by heuristics is worse and it takes longer time compared to both versions of simulated annealing.

For the small test case the solution found using simulated annealing is almost identical to the optimal solution, except that the $1 \mathrm{~V}$ and $1.1 \mathrm{~V}$ nodes are transposed. This represents a good example of a local minimum. In order to switch the two voltages two perturbations are required, the first must move either node to the correct position and the second must move the other. During the intermediate step the parent converter will not be able to meet the power requirements of both loads and will be replaced with a worse-performing converter. This degrades the overall system performance enough that the perturbation will be rejected if the temperature is too low and as a result the second perturbation to a better performing system will not be performed. The slow simulated annealing process starts with a higher temperature and cools slower, so it is able to make these transitions and find the optimal solution at the cost of backtracking when attempts to escape local minima do not work. 

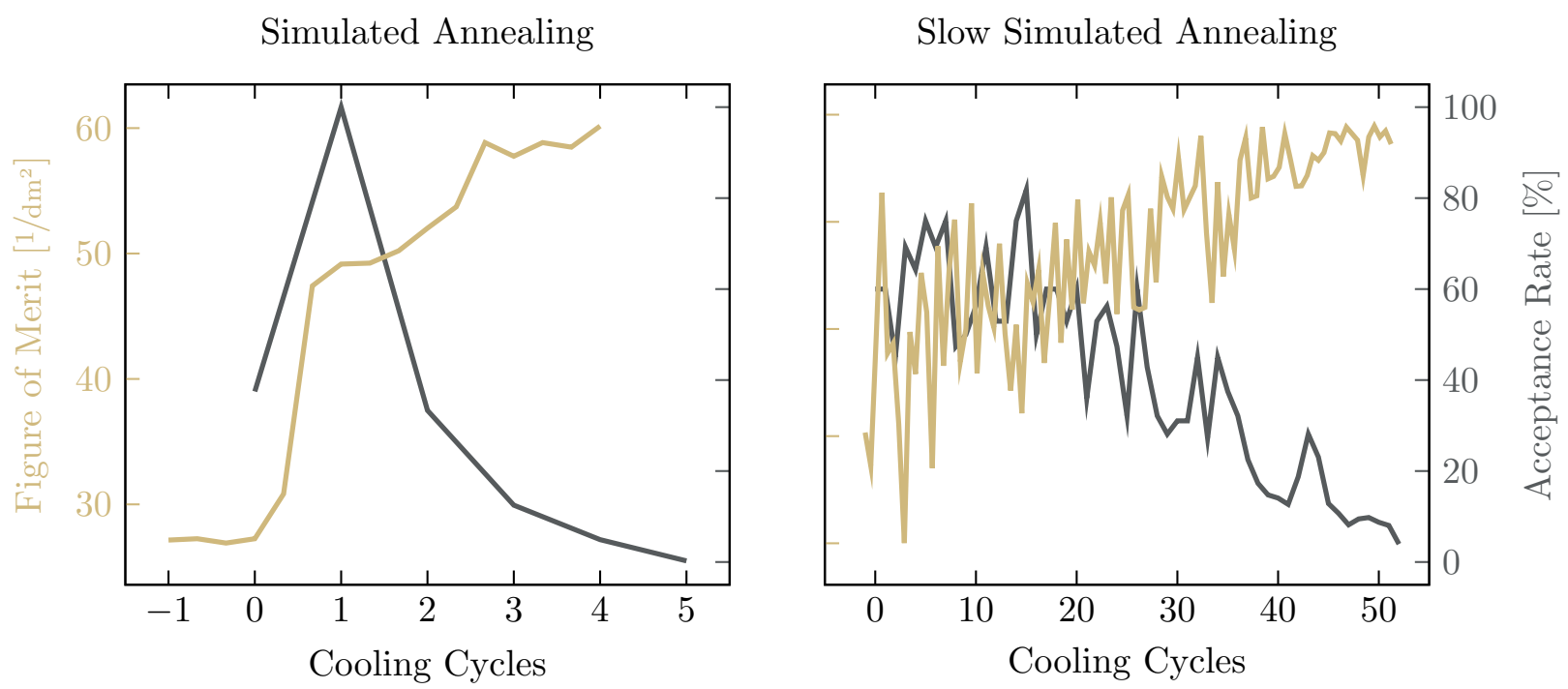

Figure 7.5: Comparison of the simulated annealing progress while solving the medium test case for the normal (left) and slow (right) versions of the algorithm.

\subsubsection{Medium Test Case}

The medium test case contains 8 output nodes, as shown in Table 6.4 and is supplied by a $48 \mathrm{~V}$ input. The optimal solution as found by the exhaustive-search and slow simulated annealing algorithms is shown in Fig. 6.9. The results in the medium case are interesting because they show how the maximum conversion ratio heuristic can fail to predict which edges may be included in the optimal system. In this system the optimal is identical to the small case except the new $0.8 \mathrm{~V}$ node is connected to the $24 \mathrm{~V}$ node. This connection has a conversion ratio of 30 which exceeds the conversion ratio of any other converter used in the system by more than a factor of two.

\subsubsection{Large Test Case}

The large test case contains 22 output nodes as shown in Table 6.5 and is also supplied by a $48 \mathrm{~V}$ input. The solution arrived at by the algorithm using simulated annealing can be seen in Fig. 7.6 and the simulated annealing progress can be seen in Fig. 7.7. In the large case there are more than $1 \times 10^{21}$ possible arrangements so the exhaustive-search method cannot be applied.

In Fig. 7.6 edges are weighted based on the log of the power they must process. This reveals some interesting power electronics insights that help explain what makes a good system solution. 
The first detail to note is that high power converters are no more than three conversion stages from the input. This is further evidence for the claim made in Section 8.3 that optimal solutions are most likely to be systems with a depth of two or three. While the optimal large solution has a depth of five, the nodes that are beyond a depth of three are relatively low power and could likely be moved such that the system depth is limited to three without significant impact to the system FOM. From this result it also appears that the maximum conversion ratio heuristic is generally a good choice; excessive conversion ratio edges are avoided and many nodes have a node similar in voltage to their own as a parent. The maximum conversion ratio is 12.9 from $12 \mathrm{~V}$ to $0.93 \mathrm{~V}$.

Finally, the results show some limitations of the "super nodes" heuristic from [41]. In the optimal system there is some correlation between the voltage of nodes that share the same parent, but the $1.8 \mathrm{~V}$ nodes, which appear three times in the system each have a different parent node. Other similar voltage nodes such as 0.85 and $0.93 \mathrm{~V}$ are also connected to different parents. It is clear that voltage alone is not necessarily a good predictor of which nodes will share a parent in the optimal system.

The progress comparison in Fig. 7.7 gives insight into how the hyperparameter selection in the two versions of simulated annealing algorithm affects the process. In the slow version the acceptance rate starts higher and moves down slowly. This causes the FOM to gradually rise from a macro point of view, but rapidly move up and down as part of the overall upward trend. In contrast, the normal version starts with a slightly lower initial acceptance ratio and rapidly brings it down as the system cools. This results in a rapid increase in the system FOM with very few decreases and those that do occur are relatively small. At the end the slow version stops as soon as the acceptance rate falls whereas the standard version continues for several more cooling cycles with only small changes while it waits for the number of stall cycles to pass. These two processes represent the tradeoff in simulated annealing between system performance and the solution time. The standard version quickly finds a good solution; however, the slow version does show that patience with local minima can yield some system performance benefits. These conclusions are also clear from Fig. 7.2 where the slow simulated annealing finds the optimal solution in all three test cases at the cost of 
2-5 times the solution time compared to the normal simulated annealing algorithm.

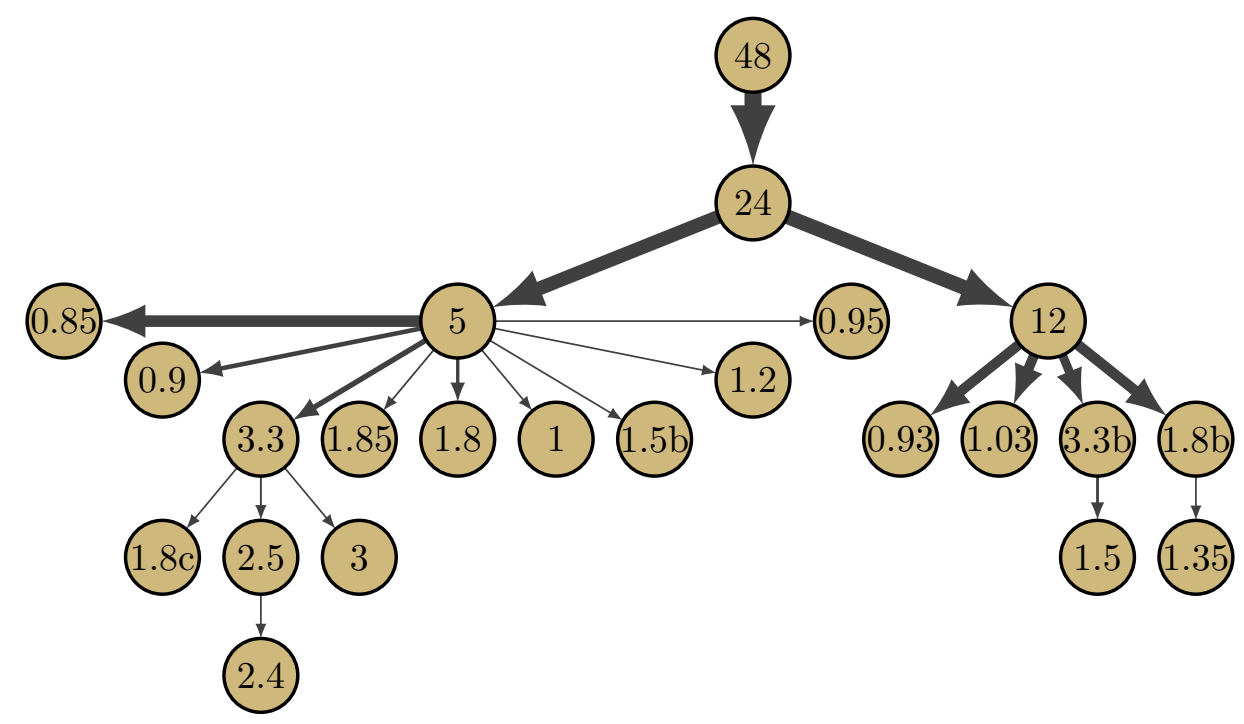

Figure 7.6: Optimal arrangement for the large test case found with simulated annealing. Edge widths are proportional to the logarithm of the power processed by the converter it represents.

\subsection{Conclusions}

Optimization of dc distribution architectures can be viewed as a combinatorial problem, which can be effectively solved using simulated annealing. This chapter describes implementation of a dc distribution optimization algorithm using simulated annealing and presents a set of test cases to compare the algorithm performance to the prior state-of-the-art including an exhaustivesearch approach and a heuristics-based approach. These test cases show that simulated annealing can produce solutions as good as or better then the prior art while reducing the solution time by up to $64 \%$ depending on the problem size and data source. Further analysis reveals insights into design heuristics that may be used by practicing design engineers. Limitations of heuristics are also discussed, showcasing the need for systematic design techniques which can improve system performance by not relying on strict design rules. 


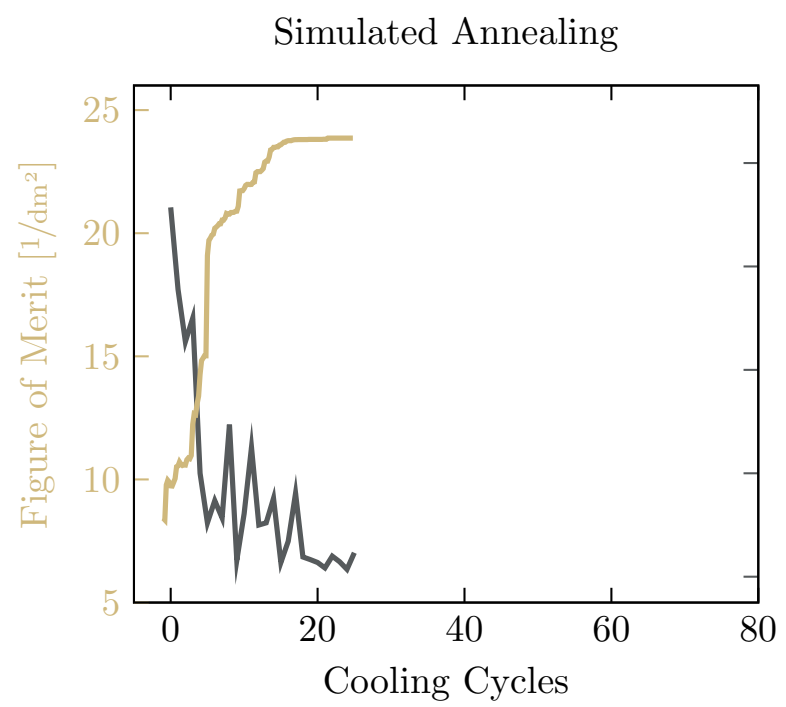

Slow Simulated Annealing

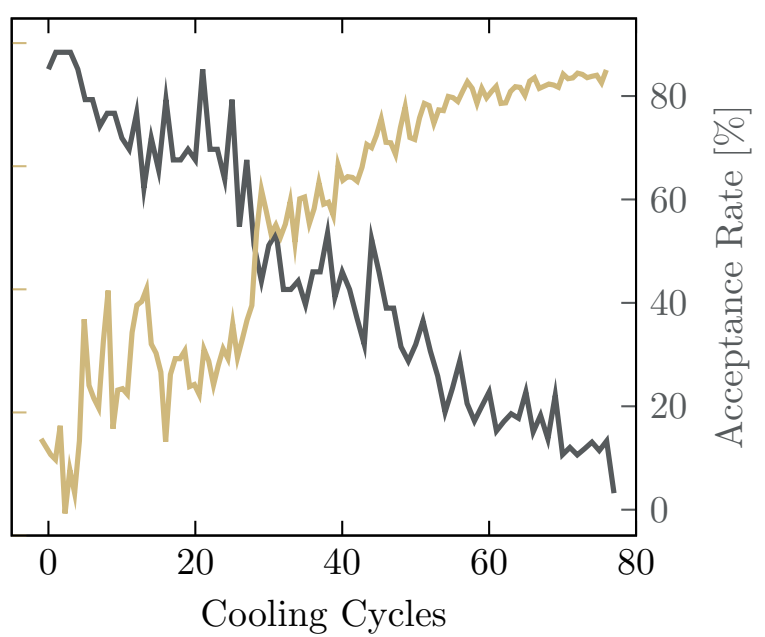

Figure 7.7: Comparison of the simulated annealing progress while solving the large test case for the normal (left) and slow (right) versions of the algorithm. In each case the initial FOM is low and the acceptance rate is high. As each algorithm progresses the FOM gradually increases and the acceptance rate decreases. In the slow case the algorithm is much more tolerant of decreases in the system FOM and there are large swings as the algorithm attempts to escape any local minima it might find. While this technique takes approximately twice the time as the normal simulated annealing approach it is able to find a slightly better solution as shown in Fig. 7.2. 


\section{Chapter 8}

\section{Case Studies}

\subsection{Accuracy of Area Estimate}

To estimate the accuracy of the area estimate, a test case is constructed and a PCB layout is manually generated, attempting to follow usual converter layout practices. For this particular test case, both the output from the WEBENCH [29] tool and the PG method are analyzed. An example layout can be seen in Fig. 8.1. Table 8.1 compares the area predicted by the two methods as well as the actual area found from the layout process. It can be seen that in both cases the area estimate is off by roughly a factor of two. This shows that while inaccurate in an absolute sense, the relative error is small, so the simple area estimate is still a good metric for comparison of architectures.

\subsection{Effect of Super Nodes}

As discussed in Section 2.4.1, the primary assumption made when reducing the number of effective nodes by adding super nodes is that voltage domains close to each other will be attached to the same parent node. However, this is not always the case. Table 8.2 shows how the FOM decreases with a reduction in the number of effective nodes for the test cases in [10]. These results

Table 8.1: Area Estimate Comparison

\begin{tabular}{llll}
\hline Tool & Area Approximation $\left[\mathrm{mm}^{2}\right]$ & Layout Area $\left[\mathrm{mm}^{2}\right]$ & Error Factor \\
\hline \hline WEBENCH & 1492 & 3160 & 2.12 \\
PG & 1532 & 2919 & 1.91 \\
\hline
\end{tabular}




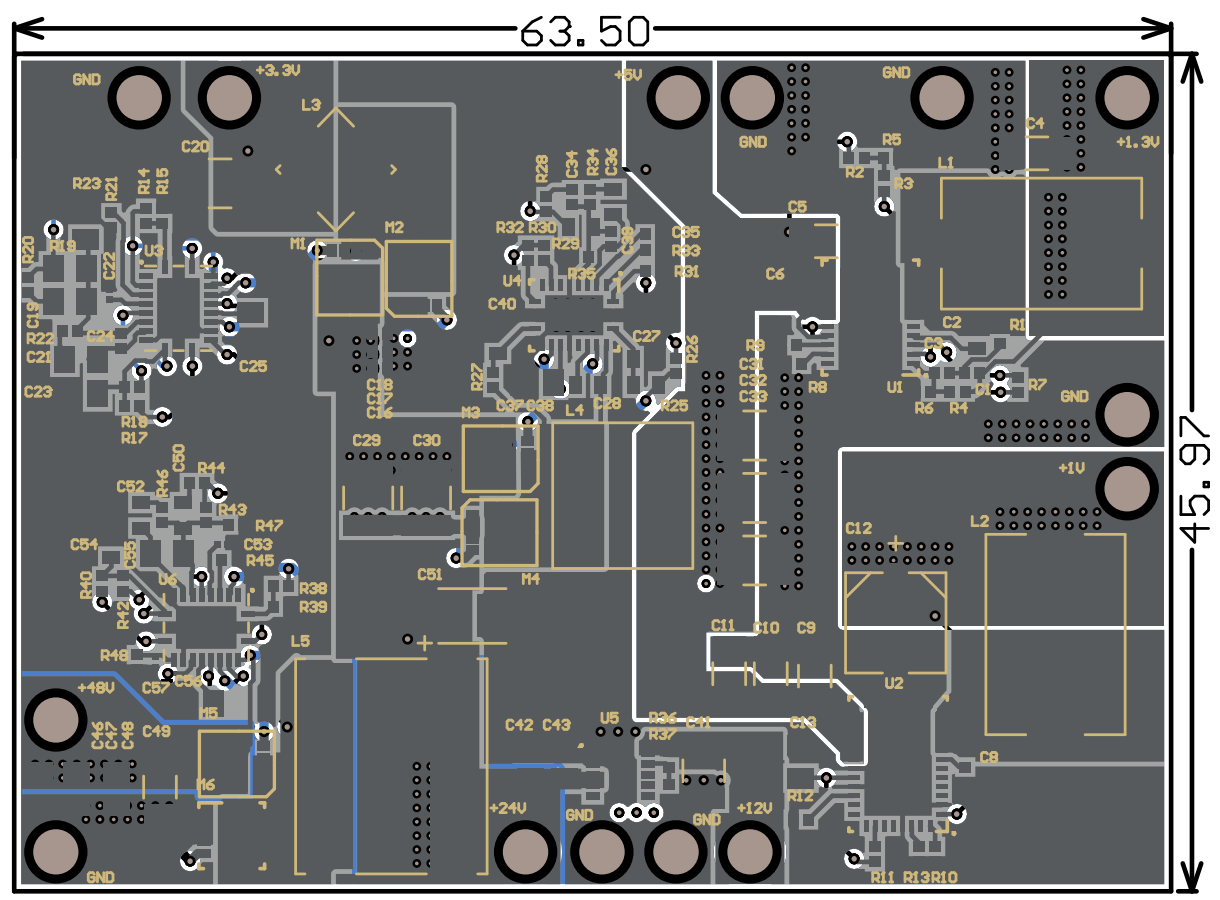

Figure 8.1: Layout of the Area Comparison Test Case. Dimensions are in mm.

match well with the expectations. When the voltage domains that are merged into a super node are close together the performance drop is relatively low; however, when voltage domains that are not close neighbors are joined into one super node the resulting system performance begins to drop dramatically. It can also be observed that there is a significant drop in the number of possibilities as the super nodes are initially formed, but that there are diminishing returns as the number of independent nodes drops.

\subsection{Effect of the Maximum Number of Cascaded Stages}

To examine the effects of depth, i.e. the maximum number of cascaded stages, the test cases in [10], which are the same as those summarized in Fig. 5.2, are re-examined in this section. First, the effect of depth on the system FOM is examined. For each test case the solutions' FOMs are normalized by the test case's optimal FOM. Finally, the solutions are separated based on their depth and the average is taken across all test cases. The results, as illustrated with grey bars in Fig. 8.2, show that the correlation between the system depth and FOM is weak. This conclusion 


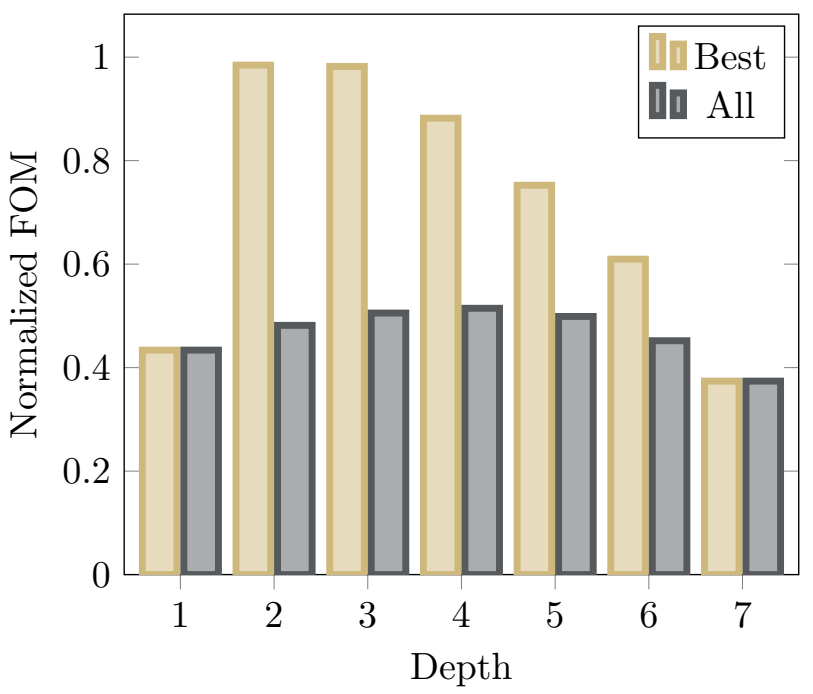

Figure 8.2: Average normalized FOM for each depth over a series of test cases. The gold-colored bars show the average of the best system at each depth while the grey bars show the average of all the arrangements at that depth. For these test cases it is very likely that the optimal solution has a depth of either two or three. Additionally, the results show that the depth alone does not correlate strongly with the FOM.

is consistent with the results in Fig. 4.1, which also implies a lack of strong correlation between architecture depth and FOM. For example, architectures with a depth of three offer simultaneously some of the best and some of the worst solutions.

Second, the likelihood that a given depth contains the overall optimal solution is examined. In this case, only the best solution at each depth is considered. The maximum FOM, averaged and normalized over all test cases, is shown as gold-colored bars in Fig. 8.2. These results show that depth is a good predictor of where the optimal designs are likely to be in the design space. For the considered test cases, the optimum is most likely to be found among architectures with a depth equal to two or three.

\subsection{Sensitivity to Converter Modeling Errors}

In order to assess the sensitivity of the algorithm to errors in the converter modeling, the test case in Fig. 8.5 is re-examined in this section. A Monte Carlo simulation is performed where the losses in all candidate converters are assumed to follow a normal distribution with a mean of 1 and 


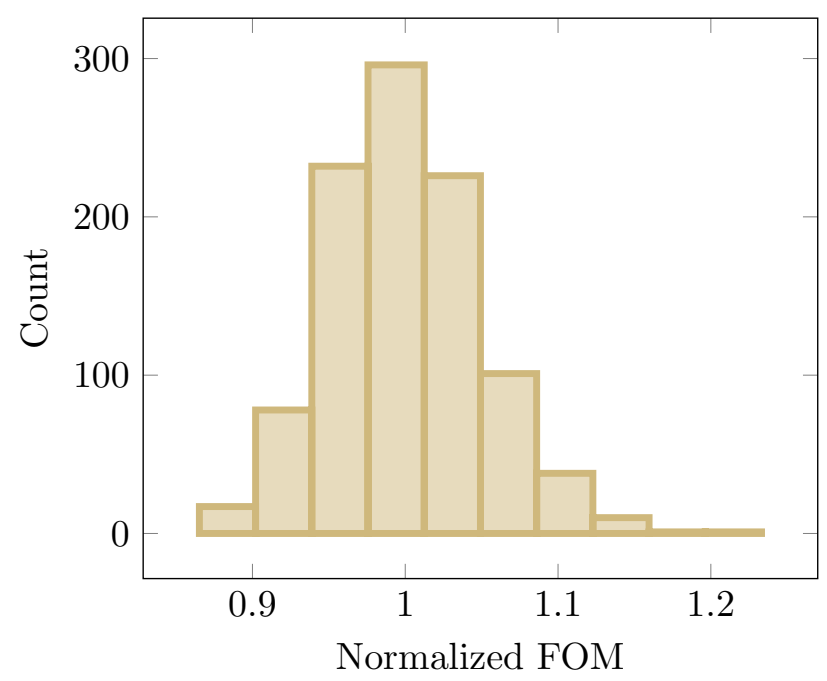

Figure 8.3: Distribution of system FOMs in a 1000 run Monte Carlo simulation assuming that errors in converter loss modeling relative to a nominal value follow a Gaussian distribution with a mean of 1 and standard deviation of 0.15 . The resulting distribution of 1000 system FOMs has a standard deviation of 0.05 , implying a relatively low sensitivity to errors in the converter loss modeling.

a standard deviation of 0.15 when normalized by the modeled losses, representing a loss modeling error. These perturbed losses are then used to select converters for each edge and to evaluate the system FOM. The results for a 1000 run simulation are shown in Fig. 8.3. Interestingly, the standard deviation of the optimal systems' FOMs is 0.05 , smaller than the input perturbation of 0.15. In $46 \%$ of the runs the optimal arrangement was the same as in the unperturbed optimal, $28 \%$ of the runs had the second most common arrangement, $11 \%$ had the third, and the remaining arrangements had fewer than $6 \%$ of the results each. Overall, the results in this test case imply a relatively low sensitivity of the system-level optimization to loss modeling errors.

\subsection{Optimal Intermediate Bus Node Placement}

While the techniques described in this thesis are primarily focused on more complex, multiple output systems, they can also be used to analyze simpler systems of practical interest. As an example, a single-input, single-output $48 \mathrm{~V}$-to- $1 \mathrm{~V}$ conversion is considered in this section at three different power levels $(20,100$, and $200 \mathrm{~W})$. The objective is to find the optimal number of intermediate bus nodes (IBN) using off-the-shelf converters from [29]. The intermediate bus voltages 


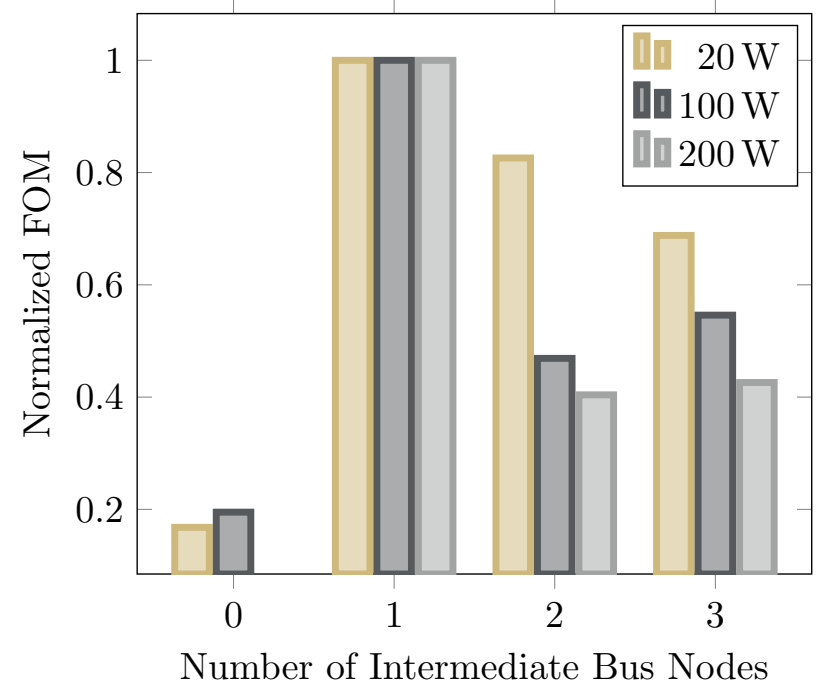

Figure 8.4: Normalized FOM vs. number of intermediate bus nodes (IBN) for 48 V-to-1 V conversion at three different power levels using off-the-shelf converters from [29]. A missing data point for the direct conversion case indicates that no converter could be found to realize the system. A single IBN results in the highest system FOM in all cases considered.

are assumed to be $12 \mathrm{~V}$ for a single IBN, $24 \mathrm{~V}$ and $5 \mathrm{~V}$ for two IBN's, and $24 \mathrm{~V}, 12 \mathrm{~V}$ and $5 \mathrm{~V}$ for three IBN's. The results in Fig. 8.4 show that a single IBN results in the highest system FOM at all three considered power levels, which is consistent with a standard practice in this application.

\subsection{Inclusion of Custom Converter Blocks}

Using the approach presented in this thesis, a second comparison is performed between the results found using WEBENCH [29], the PG method, and the PG method including custom 2:1

Table 8.2: Effect of Super Nodes on Test Cases

\begin{tabular}{|c|c|c|c|c|c|}
\hline Indel & ${ }_{\Delta}$ & 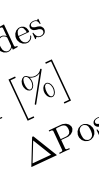 & tie $e^{50 \%]}$ & Groups & \\
\hline 6 & 0.6 & 85.7 & $40, \quad 24$, & $12, \quad 5,3.3$ & $(1.3,1)$ \\
\hline 5 & 3.3 & 97.1 & $40, \quad 24$, & $12,(5,3.3)$ & $(1.3,1)$ \\
\hline 4 & 6.7 & 99.5 & $40, \quad 24$, & $12, \quad(5,3.3$ & $1.3,1)$ \\
\hline 3 & 30.1 & 99.9 & $40, \quad 24$, & $(12, \quad 5,3.3$ & $1.3,1)$ \\
\hline 2 & 56.6 & 100 & $40,(24$, & $12, \quad 5,3.3$ & $1.3,1)$ \\
\hline
\end{tabular}




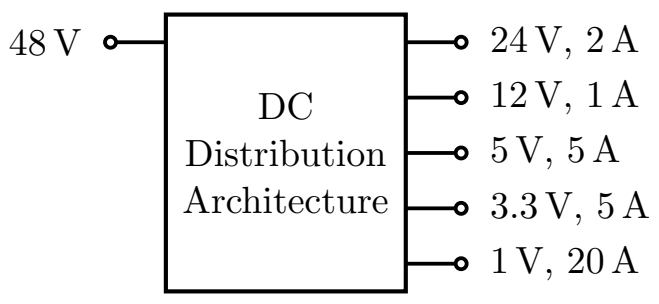

Figure 8.5: The DC distribution architecture for the case study described in Section 8.6. The architecture is realized using two different arrangements of dc-dc converter modules, one using only COTS blocks and another using COTS and custom TSAB blocks.

converters for the test case shown in Fig. 8.5. The custom 2:1 converters, which follow the approach described in [39], belong to the family of transformerless stacked active bridge (TSAB) converters [40], which feature small inductor sizes and very high efficiency when operated around a nominal conversion ratio. The TSAB converter schematic and design details can be seen in Fig. 8.6. Additionally, a fourth case is considered, where the optimal system found with the PG method is modified to use the TSAB converters as drop-in replacements, but with no change in the arrangement.

The system optimization results can be seen in Table 8.3. Restricted to just standard converter choices, the PG method offers a $53 \%$ improvement over WEBENCH. With the inclusion of custom 2:1 TSAB converters this improvement over WEBENCH increases to $80 \%$.

The optimum arrangement for the case when only standard converters are available can be seen in Fig. 1.4a. When the custom 2:1 TSAB converters are added to the pool of available choices,

Table 8.3: Model Based System Comparison

\begin{tabular}{lllll}
\hline Method & $\rho\left[\mathrm{W} / \mathrm{cm}^{2}\right]$ & $\eta[\%]$ & FOM $\left[1 / \mathrm{dm}^{2}\right]$ & $\Delta \mathrm{FOM}$ \\
\hline \hline WEBENCH [29] & 8.14 & 90.1 & 61.0 & \\
PG COTS Only & 9.36 & 92.4 & 93.4 & $53 \%$ \\
2:1 Drop-in & 8.20 & 94.0 & 95.9 & $57 \%$ \\
PG COTS + 2:1 & 8.87 & 94.3 & 109.7 & $80 \%$ \\
\hline
\end{tabular}

Model-based comparison of four different methods of designing the system shown in Fig. 8.5. The "2:1 Drop-in" method replaces the 2:1 COTS converters ( $48 \mathrm{~V}$ to $24 \mathrm{~V}$ and $24 \mathrm{~V}$ to $12 \mathrm{~V}$ ) in the PG COTS system with custom TSAB 2:1 converters without altering the arrangement. 


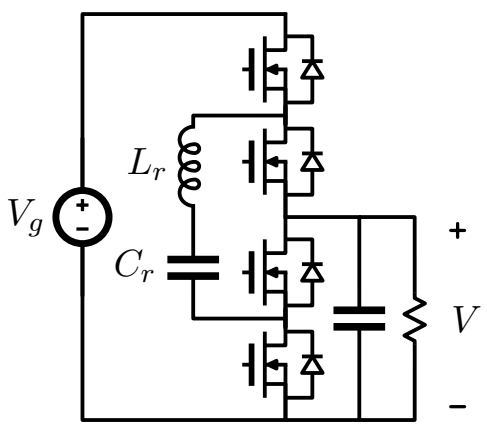

Figure 8.6: Custom 2:1 TSAB converter [39], [40]. One of the hardware system prototypes contains two of these converters, as shown in Fig. 8.7b. The first custom TSAB module operates from $48 \mathrm{~V}$ to $24 \mathrm{~V}$ and uses $40 \mathrm{~V}$ EPC2024 eGaN FETs operating at $150 \mathrm{kHz}$, with $C_{r}=15 \mu \mathrm{F}$ and $L_{r}=810 \mathrm{nH}$ planar inductor made with an ELT 11 core. The second custom TSAB module operates from $24 \mathrm{~V}$ to $12 \mathrm{~V}$ and also uses $40 \mathrm{~V}$ EPC2024 eGaN FETs operating at $150 \mathrm{kHz}$ with $C_{r}=28 \mu \mathrm{F}$ and $L_{r}=360 \mathrm{nH}$ planar inductor made with an ELT 11 core. Both converters have modeled efficiency of $99 \%$ at their nominal operating point (121.5 W and $73.5 \mathrm{~W}$ respectively.) The cascaded efficiency of the two modules was measured at $98 \%$.

the optimum arrangement changes to that of Fig. 1.4b. It can be seen from these results that new converter topologies can have a significant impact on the optimal arrangement and the system FOM. Furthermore, it can be seen from the drop-in results that the improvements in the system FOM are not caused by the TSAB converters alone. A drop-in replacement in the arrangement found using COTS-only converters result in only a modest increase in the system FOM. Looking at these results together it can be seen that the improved converters account for only $15 \%$ of the system FOM whereas the other $85 \%$ can be attributed to the change in arrangement.

In general, with more efficient topologies available, multiple voltage domain power distribution systems can be rearranged to allow converters supplying lower voltage domains with smaller conversion ratios. These results demonstrate that improved converters not only allow for improvements to individual blocks in a power distribution architecture, but also enable structural changes to the architecture, which can result in more substantial system level improvements. Furthermore, improved topologies lead to a shift in the Pareto front, as seen in Fig. 8.8. 


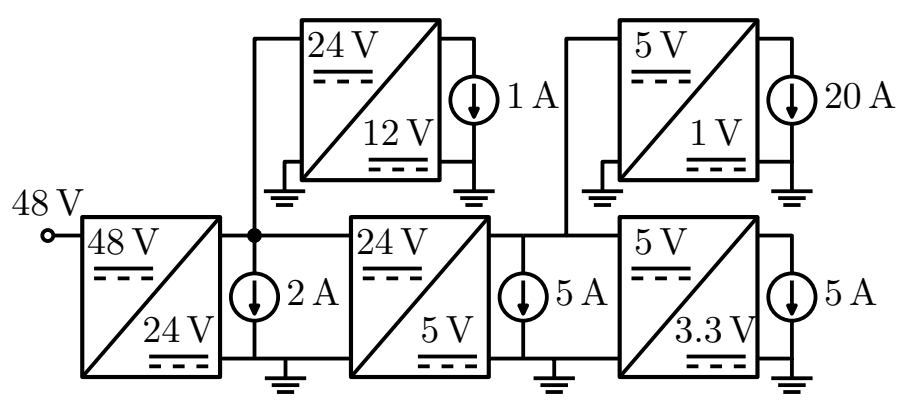

(a) COTS Only

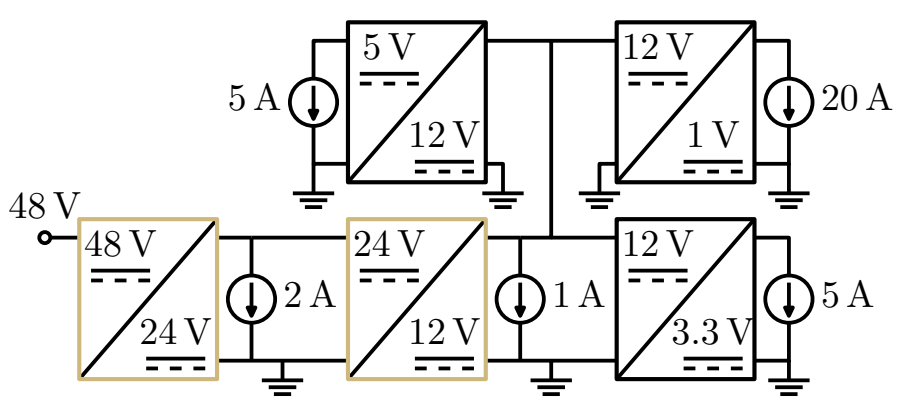

(b) COTS \& TSAB

Figure 8.7: Block diagrams for the system prototypes. (a) shows the system containing only COTS converter blocks, an image of which can be seen in Fig. 9.1a. (b) shows the system containing the custom 2:1 TSAB blocks as well as the COTS blocks. In this system the $48 \mathrm{~V}$ to $24 \mathrm{~V}$ converter as well as the $24 \mathrm{~V}$ to $12 \mathrm{~V}$ converter were implemented using the 2:1 TSAB converters shown in Fig. 8.6. An image of this system can be seen in Fig. 9.1b. (a) and (b) are block diagram representations of the graphs shown in Fig. 1.4a and Fig. 1.4b respectively. 


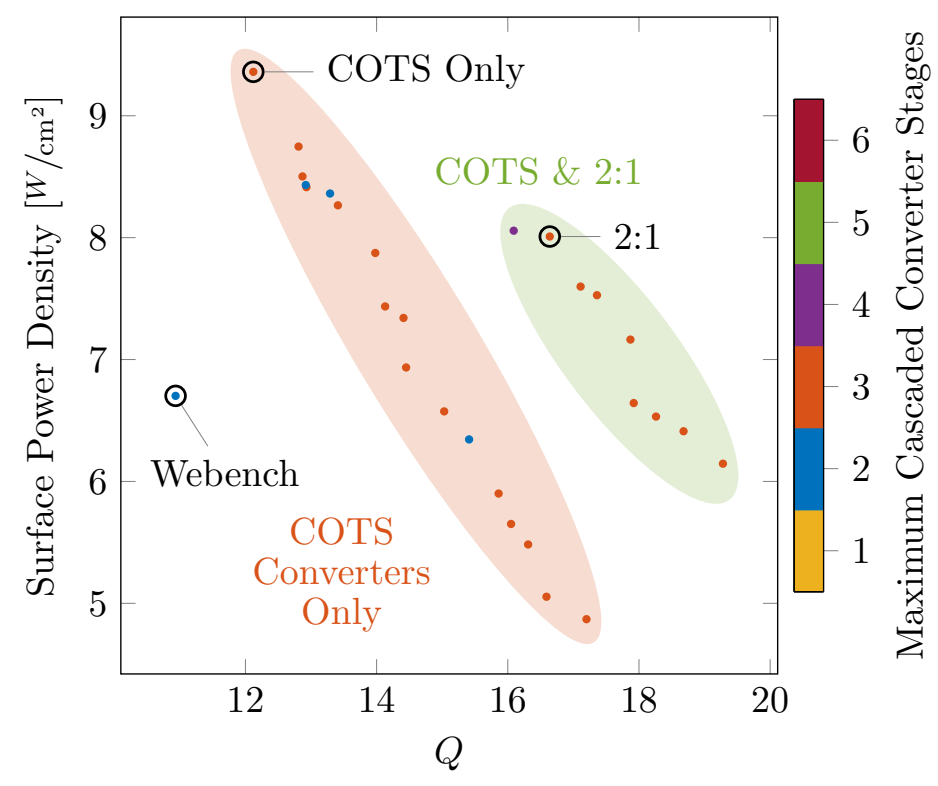

Figure 8.8: Surface density vs. quality factor $Q$ Pareto fronts generated algorithmically as in Fig. 4.2 for two cases: systems using only standard COTS converters and systems also using custom 2:1 converters. In each case converters are selected to meet the required input and output voltage and current requirements, and to maximize each edge's figure of merit as defined in (4.2). Called out data points represent the systems that achieve the highest FOM for three cases: the system recommended by WEBENCH $\left(\mathrm{FOM}=61 \mathrm{dm}^{-2}\right)$, the highest FOM system obtained by the PG method using COTS modules only $\left(\mathrm{FOM}=93 \mathrm{dm}^{-2}\right)$, and the highest FOM system obtained by the PG method using COTS and custom 2:1 converters $\left(\mathrm{FOM}=110 \mathrm{dm}^{-2}\right)$. System comparison details are shown in Table 8.3. 


\section{Chapter 9}

\section{Experimental Results}

Two experimental prototypes have been constructed from the test case examined in Section 8.6. The system built with only Commercial Off The Shelf (COTS) converter blocks is shown as a block diagram in Fig. 8.7a and a prototype photo is shown in Fig. 9.1a. The version using custom 2:1 TSAB and COTS converter blocks is shown as a block diagram in Fig. 8.7b and a prototype photo is in Fig. 9.1b. For the two prototype systems converter blocks using COTS components were built to manufacturer specifications and the two TSAB converters were built with specifications detailed in Fig. 8.6.

To test the two systems a custom electronic load is used; a block diagram of the setup can be seen in Fig. 9.6 and a photo of the full experimental setup can be seen in Fig. 9.2. The multiple-channel programmable load uses power MOSFETs operating in the linear region as current sources with gate voltages set by a feedback loop using references from a microcontroller. Input power is measured with a voltage measurement on each power board (Figs. 9.1a and 9.1b) and a current measurement in-line with the input power supply. Output power is measured with voltage measurements for each output on the power boards and current measurements using sense resistors on the load board. The experimental results are shown in Table 9.1 along with measurement details in Tables 9.2 to 9.4. These results show that the hardware prototype that includes the 2:1 TSAB blocks achieves a $179 \%$ increase in system figure of merit over the system that contains only Commercial Off The Shelf (COTS) converter blocks by achieving significantly higher system efficiency with comparable power density.

A comparison between model-based results and the experimental results reveals some dis- 


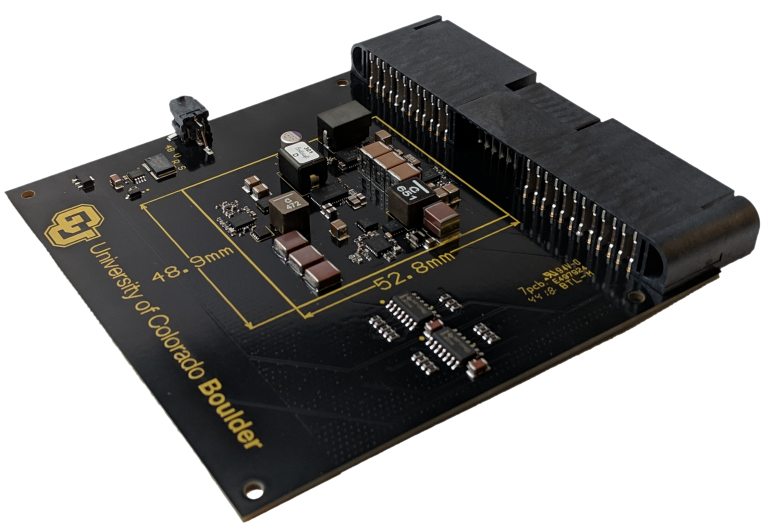

(a) COTS Only

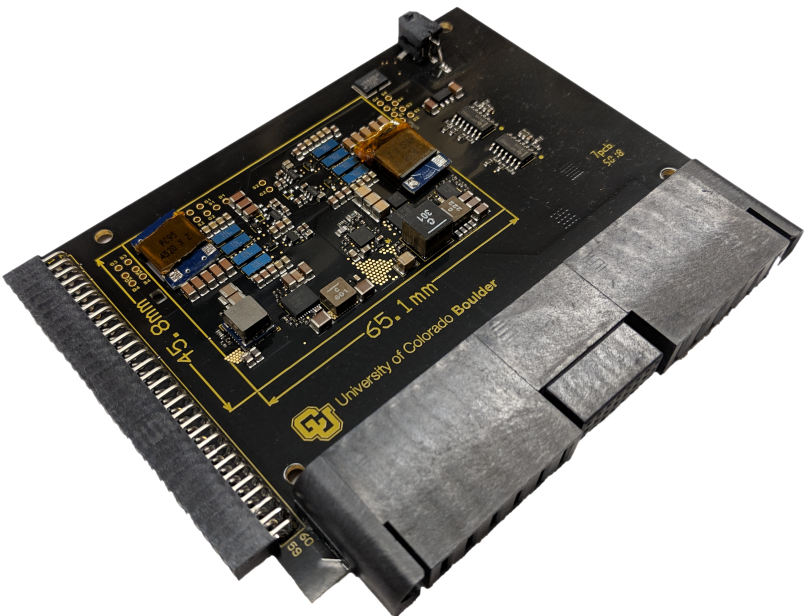

(b) TSAB \& COTS

Figure 9.1: Hardware prototypes of the case study in Fig. 8.5 (a) using only COTS converter blocks, as shown in a block-diagram form in Fig. 8.7a and (b) using COTS and custom 2:1 TSAB converter blocks, as shown in Fig. 8.7b.

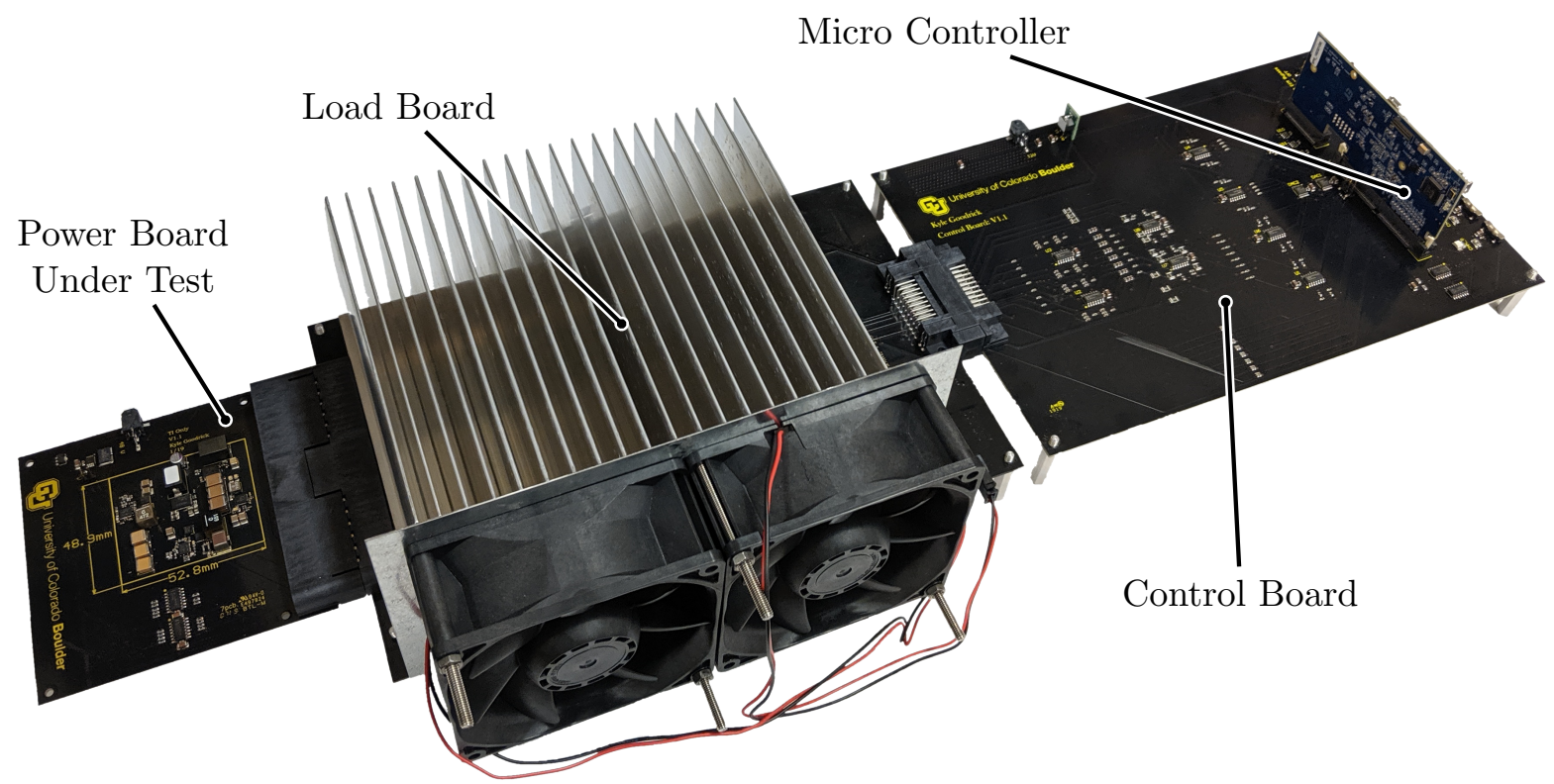

Figure 9.2: Experimental test setup

Table 9.1: Experimental System Comparison

\begin{tabular}{llllll}
\hline Method & Arrangement & $\rho\left[\mathrm{w} / \mathrm{cm}^{2}\right]$ & $\eta[\%]$ & FOM $\left[1 / \mathrm{dm}^{2}\right]$ & $\Delta$ FOM \\
\hline \hline PG COTS Only & Fig. 1.4a & 4.71 & 87.7 & 27.6 & \\
PG $+2: 1$ & Fig. 1.4b & 3.80 & 96.1 & 77.0 & $179 \%$ \\
\hline
\end{tabular}




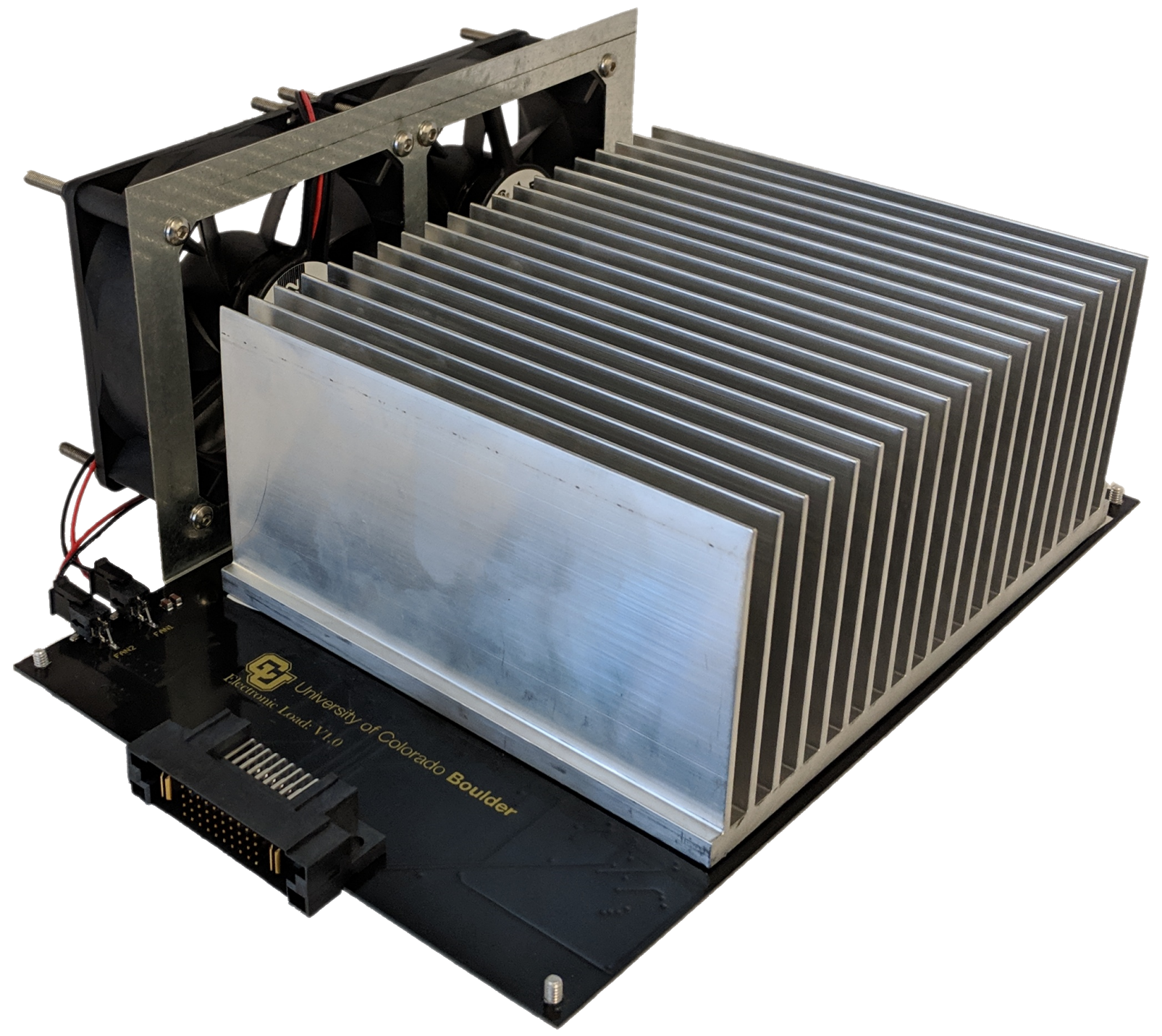

Figure 9.3: Load Board 


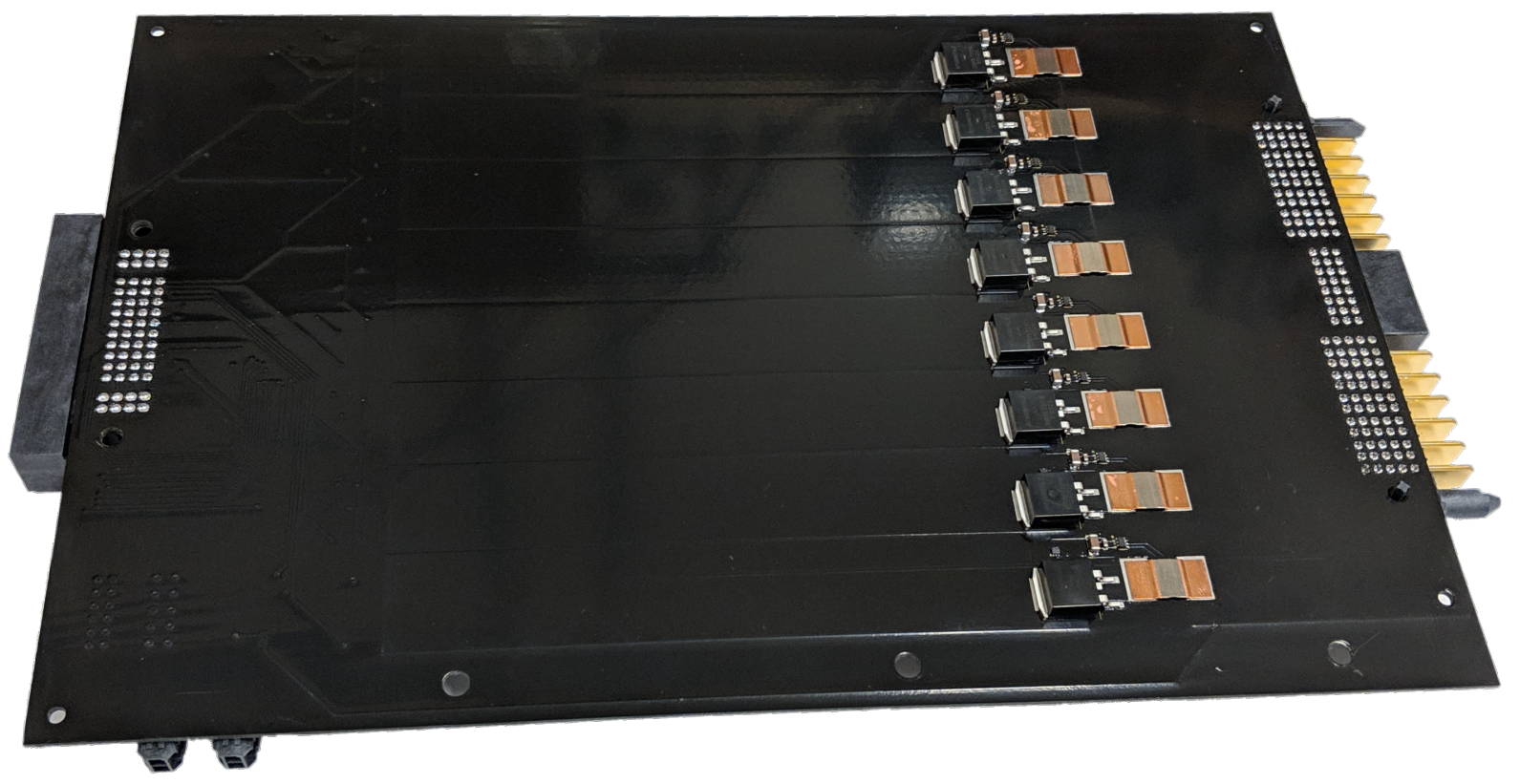

Figure 9.4: Load Board Bottom

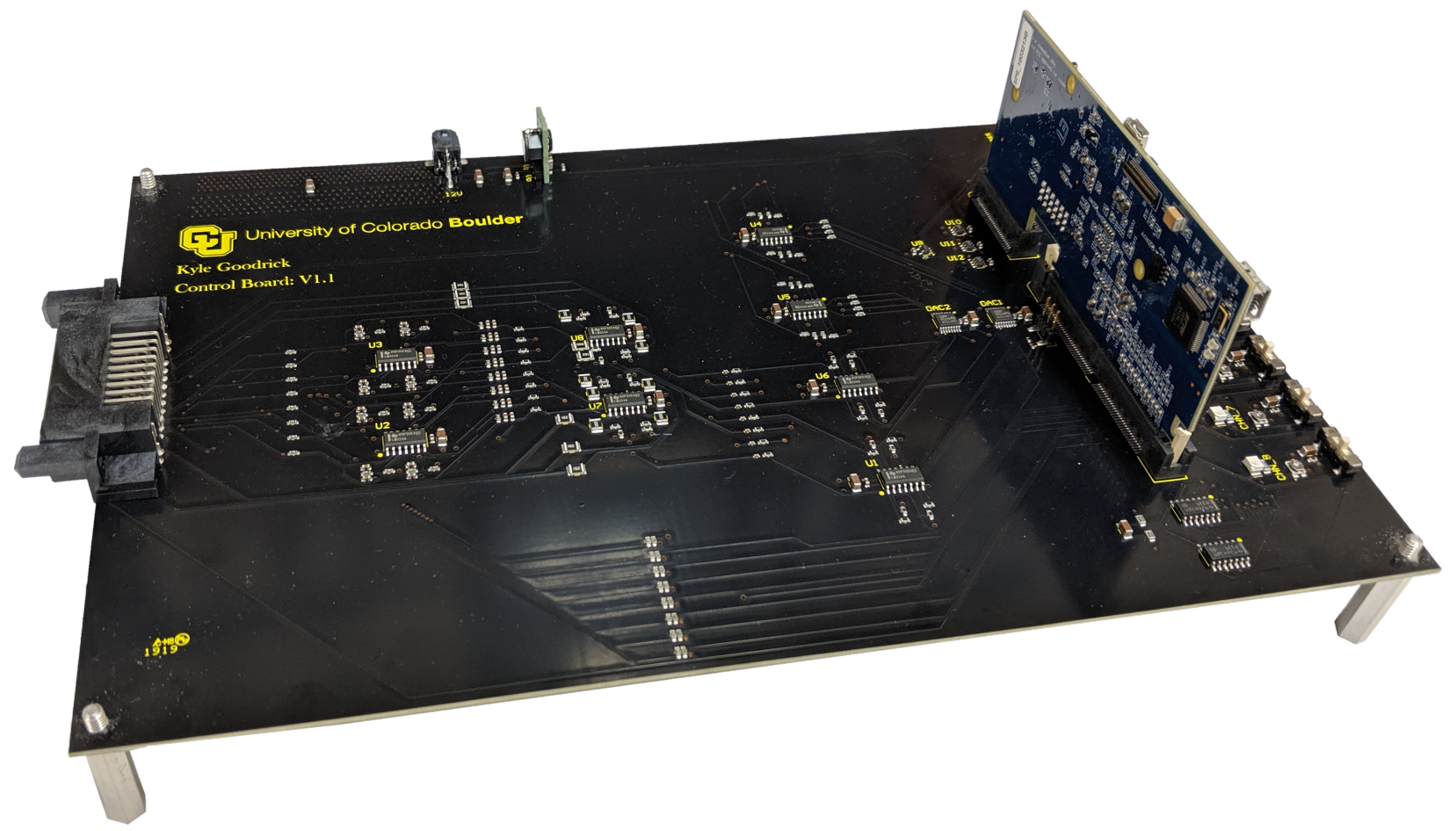

Figure 9.5: Control Board 


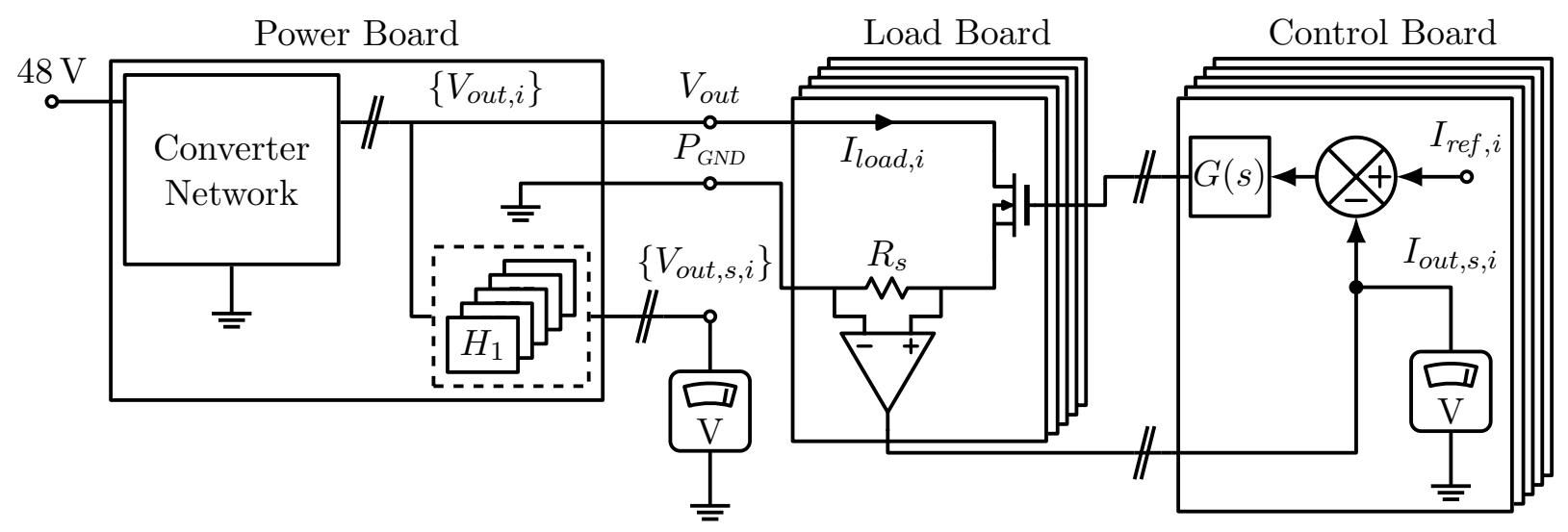

Figure 9.6: The experimental setup. $48 \mathrm{~V}$ is provided to the power board prototype (Fig. 8.7a or Fig. 8.7b), which then generates the required output voltages, $\{24,12,5,3.3$, and 1$\}$ V. Voltage measurements are also performed on the power board. The power board connects to the custom programmable load board, which has multiple channels, one for each output. Load currents are sensed on the load board, amplified and sent to the control board. The control board includes current measurements and closes the feedback loop to regulate the load currents to the specified reference values.

crepancies. Most importantly, the power density of experimental prototypes is smaller compared to the models. As mentioned in Chapter 4 and presented in more detail in [10], the model employs a standard area estimation method where the area for each converter is found as the area of each component in the bill of materials with $1 \mathrm{~mm}$ of extra space on each side. This results in an underestimate of approximately half the real prototype area as shown in [10]. Nevertheless, when applied uniformly, this method provides a way to fairly compare different converters in the optimization algorithm. Differences between predicted and actual system efficiencies can be attributed to characterization issues in the TI Webench database. Nevertheless, the experimental results demonstrate FOM improvement trends consistent with the model predictions. 
Table 9.2: Detailed Experimental Results - COTS Only

\begin{tabular}{ccccc}
\hline \multicolumn{2}{c}{ Output } & Voltage [V] & Current [A] & Power [W] \\
\hline \hline 24 & $\mathrm{~V}$ & 24.13 & 1.99 & 48.11 \\
12 & $\mathrm{~V}$ & 12.02 & 1.01 & 12.18 \\
5 & $\mathrm{~V}$ & 4.91 & 5.04 & 24.75 \\
$3.3 \mathrm{~V}$ & 3.30 & 5.05 & 16.66 \\
1 & $\mathrm{~V}$ & 0.97 & 20.23 & 19.56 \\
\hline
\end{tabular}

Table 9.3: Detailed Experimental Results — TSAB \& COTS

\begin{tabular}{ccccc}
\hline \multicolumn{2}{l}{ Output } & Voltage [V] & Current [A] & Power [W] \\
\hline \hline 24 & $\mathrm{~V}$ & 24.51 & 2.03 & 49.68 \\
12 & $\mathrm{~V}$ & 12.07 & 0.99 & 11.95 \\
5 & $\mathrm{~V}$ & 4.99 & 5.05 & 25.19 \\
$3.3 \mathrm{~V}$ & 3.28 & 5.06 & 16.61 \\
1 & $\mathrm{~V}$ & 0.97 & 20.24 & 19.70 \\
\hline
\end{tabular}

Table 9.4: Summary Measurements

\begin{tabular}{clll}
\hline & & COTS Only & TSAB \& COTS \\
\hline \hline$P_{\text {out }}$ & {$[\mathrm{W}]$} & 121.25 & 123.12 \\
$V_{\text {in }}$ & {$[\mathrm{V}]$} & 47.59 & 47.59 \\
$I_{\text {in }}$ & {$[\mathrm{A}]$} & 2.91 & 2.69 \\
$P_{\text {in }}$ & {$[\mathrm{A}]$} & 138.29 & 128.17 \\
$\eta$ & {$[\%]$} & 87.7 & 96.1 \\
\hline
\end{tabular}




\section{Chapter 10}

\section{Power Electronics Performance Models}

In the field of power electronics machine learning has been used for control [55], [56] and fault detection [57]-[61], but has seen relatively less focus on the application of Artificial Intelligence (AI) to the design phase [62]-[64].

This chapter is focused on applications of machine learning (ML) techniques in the design of complex power electronics systems consisting of multiple dc-dc converters. In such systems, there are often many ways to configure the system architecture and to choose how the converter modules are implemented [41]. In such cases, it is challenging and computationally expensive to perform detailed characterization of many possible converter realizations. Consider, for example, systems where a large step-down dc-dc conversion is required, such as server boards where a low point-of-load voltage, e.g. $1 \mathrm{~V}$ is generated starting from an input dc bus voltage, e.g. $48 \mathrm{~V}$. In such applications, one option is to implement the large step down using two or more cascaded converters, with IBNs [24], [25] in between. The placement of IBNs, and the choice of the converter modules can be considered a complex, computationally expensive optimization problem since there are many possible choices available for the intermediate bus voltages and the converters. This chapter shows how ML based dc-dc converter performance models can be constructed and used to efficiently complete system optimization tasks.

This chapter is structured as follows: first Section 10.1 gives an introduction to the dc-dc converter performance model and its goals and requirements, then Section 10.2 compares various machine learning approaches applied to dc-dc converter performance models. Next Section 10.3 provides a more detailed look at the chosen machine learning techniques and finally Section 10.4 
details applications of the dc-dc converter performance model and the performance of the presented models.

\subsection{DC-DC Converter Performance Model}

The dc-dc converter performance model has certain input and output requirements that must be fulfilled as shown in Fig. 10.1. The system's input and output voltages as well as output current are given as inputs to the estimator. The estimator must then make several predictions: first, the estimator should classify the inputs as feasible or infeasible. There are certain converter input and output specifications that are not feasible and the estimator should clearly state these limits. Second, the estimator must make a series of regression based predictions: area, losses, cost, and FOM if required by the application. To accomplish both of these goals the process is split into two tasks - classification and regression — that are each handled by a separate ML based estimator.

In this work the data to train the the performance model comes from the TI WEBENCH design tool [29] accessed via automated web scraping. The WEBENCH design tool takes input and output voltages along with input current as inputs and outputs a list of converters that can realize the system. This list contains information about each solution such as efficiency, maximum output current, BOM Area, BOM Cost, and BOM Count. This list is more sophisticated than a database as the efficiency values are computed for the specified operating point and results change as individual components stock and price vary. It is also possible to ask the tool to design converters with specific optimization goals in mind: high efficiency, small footprint, low cost, or a balance of all three.

For dc-dc converter modeling there are a few key considerations when accessing data from WEBENCH and using it to train the models. First, the optimization goal is important and the model outputs will change depending on the goals of the application. When optimizing for area predictions for area will be smaller than when optimizing for efficiency. Second, the goal of the model is not to predict the average converter available on WEBENCH, but the one that would be used in the current application. After all data has been collected from WEBENCH (Perhaps for 


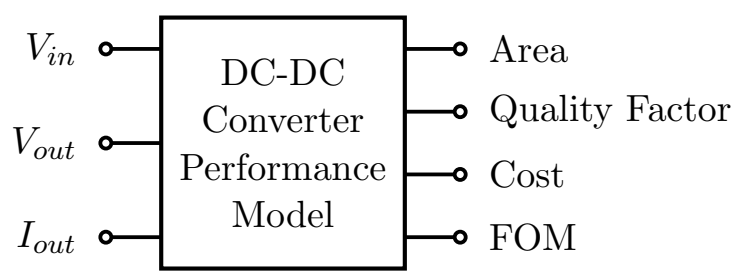

Figure 10.1: Inputs and outputs for the DC-DC converter performance model. $V_{\text {in }}, V_{\text {out }}$, and $I_{\text {out }}$ are supplied and the estimator predicts the performance characteristics of interest.

more than one WEBENCH optimization goal) the converters are evaluated and the one that best meets the goals of the application is chosen. Only the properties of this converter should be used to train the performance model. Finally, only properties available on WEBENCH may be estimated. It is not possible to estimate properties that WEBENCH does not return, for example the height (and therefore the volume) or the weight of the converter.

\subsection{Comparison of Machine Learning Estimators for Converter Perfor- mance}

To compare the performance of different machine learning estimation techniques a sample of 1000 converters is drawn from the TI WEBENCH tool [29]. There is nothing limiting the technique to commercially available converters; however, the use of COTS converters is widespread and data for their performance is readily available. First, input voltages are generated with a uniform distribution between $0.6 \mathrm{~V}$ to $70 \mathrm{~V}$, then output voltages are generated with a uniform distribution from $0.5 \mathrm{~V}$ to the input voltage, and finally an output power is generated from a uniform distribution between $0 \mathrm{~W}$ to $100 \mathrm{~W}$ and the output current is computed. Once the input data has been generated, WEBENCH [29] is used to retrieve the output data on efficiency, size,

Table 10.1: Classification Results

\begin{tabular}{ll}
\hline Estimator & Specificity \\
\hline \hline Gradient Boosting [65] & 0.80 \\
Ada Boost [66] & 0.72 \\
Random Forest [67] & 0.71 \\
SVC [68] & 0.25 \\
\hline
\end{tabular}




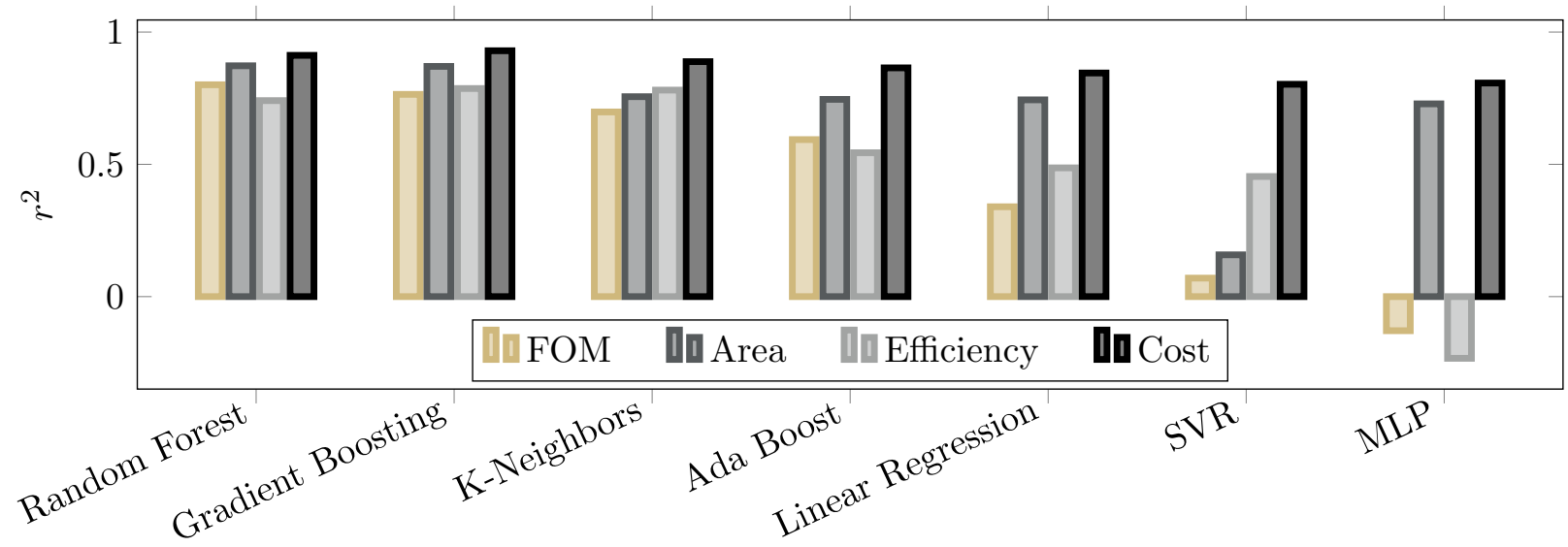

Estimator Type

Figure 10.2: Performance comparison for different estimator techniques for varying optimization goals. Random Forest has the best coefficient of determination when optimizing for FOM and among the best when optimizing for other attributes. Negative values of $r^{2}$ indicate that the estimate is worse than an estimator that always predicts the mean.

cost, and feasibility. Once data for efficiency is retrieved it is used to compute quality factor

$$
Q=\frac{\eta}{1-\eta}=\frac{P_{\text {out }}}{P_{\text {loss }}}
$$

in order to create more separation between dissimilar values. All estimators are implemented using Scikit-Learn [69] using the default hyperparameters.

\subsubsection{Classification}

For classification the data is heavily imbalanced - only 53 of the 1000 data points are infeasible — which would give a classifier that always predicts feasibility an accuracy of nearly $95 \%$. Therefore, instead of accuracy, specificity is used to evaluate classifiers to ensure that classification of infeasible results is the most important factor. Specificity is defined as

$$
\text { Specificity }=\frac{\mathrm{TN}}{\mathrm{TN}+\mathrm{FP}}
$$

where TN is the number of true negatives (infeasible predicted as infeasible) and FP is the number of false positives (infeasible predicted as feasible.) Specificity results for four classification techniques can be found in Table 10.1 which shows acceptable results for Gradient Boosting [65], 


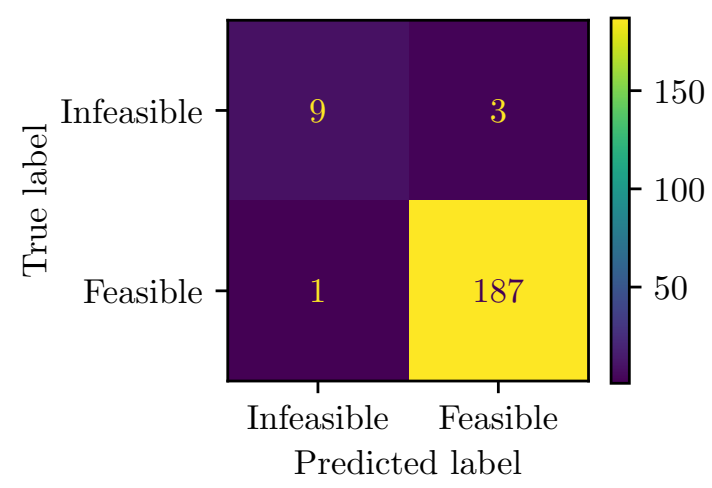

Figure 10.3: Confusion matrix for gradient boost classifier trained on 800 data points and tested on 200. Results show that of 12 infeasible inputs 9 are classified correctly with only one misclassified feasible solution.

Ada Boost [66], and Random Forest [67] classifiers, but poor performance with support vector classifiers [68].

\subsubsection{Regression}

For regression different optimization goals must be considered separately. Estimators should output different areas and efficiencies when optimizing for different goals. A variety of estimator techniques are compared (Random Forest [67], Gradient Boosting [65], K-Nearest-Neighbors, Ada Boost [66], Linear Regression, SVR [68], and MLP [70]). The results summarized in Fig. 10.2 show good performance for random forests [67] and gradient boosting [65] approaches. Fig. 10.2 shows how each technique performs when optimizing for each optimization goal. In each case the estimator predicts all properties (efficiency, area, cost, FOM) but the property of interest is the only prediction that affects the estimator's $r^{2}$ performance.

\subsection{Detailed Examination of the Techniques Selected for Classification and Regression}

The performance comparisons show that gradient boosting performs best for classification and random forests best for regression and each warrant deeper examination. The full test prediction 


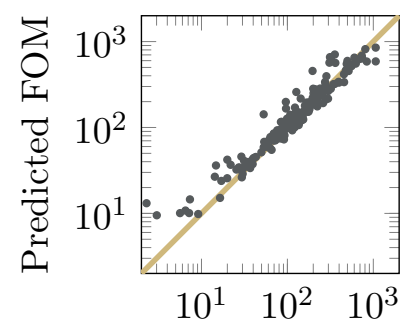

True FOM

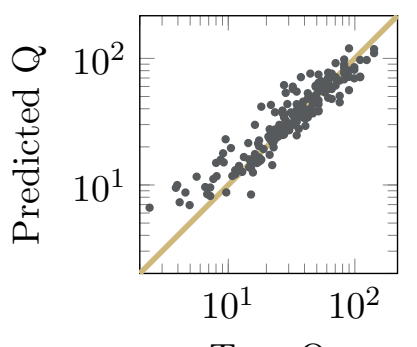

True Q

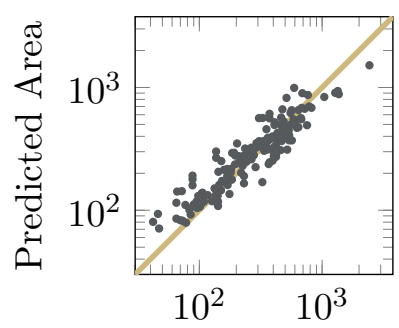

True Area

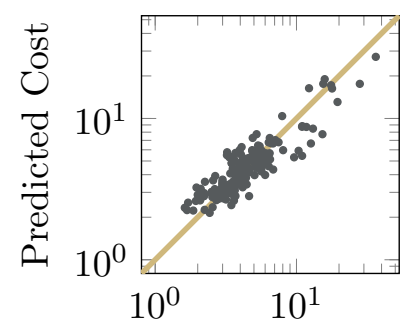

True Cost

(a) Optimizing for FOM

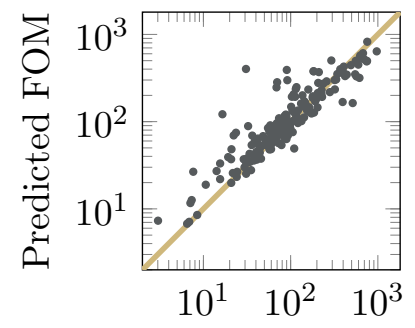

True FOM

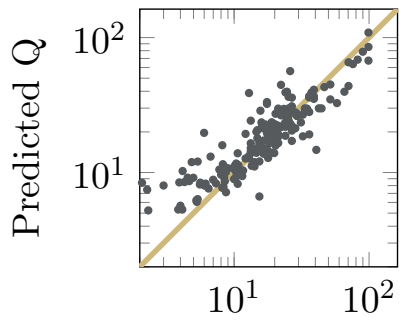

True Q

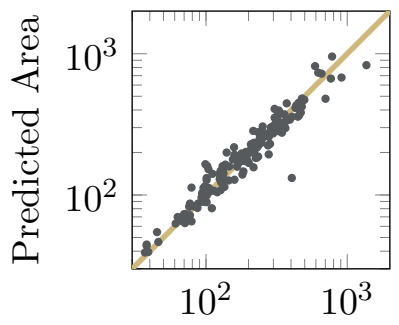

True Area

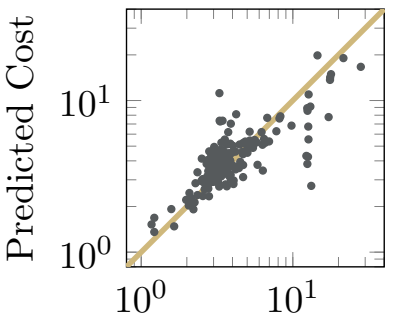

True Cost

(b) Optimizing for Area

Figure 10.4: Comparison between real and predicted values for 200 test data points. Estimators are both random forests trained on 800 data points and are optimizing for FOM (a) and area (b). Results show that across a wide range of FOM and area values the estimators are able to predict true FOM or area with a reasonable accuracy. The non-optimized properties are also well approximated by the estimators; however, it is clear that the estimators' most accurate predictions are for their respective optimization goal.

results for the gradient boosting classifier is shown in Fig. 10.3. These results show that the classifier correctly classifies all but one feasible result and misclassifies only 3 of 12 infeasible converters.

Detailed results for the regressor can be seen in Fig. 10.4 which shows the comparison between true and predicted values for FOM, footprint area, quality factor, and BOM cost for a random forest estimator optimizing for FOM in Fig. 10.4a and Area in Fig. 10.4b. These results show that when optimizing for FOM predictions for FOM are within a constant percentage tolerance band throughout the range of possible FOMs. Predictions for area, quality factor, and cost are also reasonably close to their true value. Fig. 10.4b shows the same plot, but for an estimator trained on converters optimized for area. The results here are very similar to those when optimizing for FOM, but the most accurate predictions are for area. Similar results are seen when optimizing for the 
other properties, efficiency and cost. The estimators predict quality factor $Q$ instead of efficiency as efficiencies of power converters are concentrated within a much narrower band than quality factors, making prediction more difficult. A test case shows that for random forest estimators this transformation improves predicted $r^{2}$ from 0.68 to 0.73 .

Additionally, performance of the estimator along typical curves can be seen in Fig. 10.5. In these results estimators generate results along sweeps of typical converter operating points. The results show that the estimator is capable of following the trends as the input voltage, output voltage, and output power are varied. This includes following highly nonlinear trends in the FOM such as in Fig. 10.5b where the 48-24 V converter has a sharp decline in the FOM when the power exceeds approximately $15 \mathrm{~W}$ or in Fig. $10.5 \mathrm{f}$ where there is a rapid increase in the FOM when the converter with a $24 \mathrm{~V}$ input has an output that exceeds approximately $12 \mathrm{~V}$ followed by a decrease around $17 \mathrm{~V}$ output.

\subsubsection{Feature Engineering}

In addition to the features required to make the predictions for converter performance it is also possible to generate or "engineer" new features that can be used to improve the performance of the estimator. These engineered features are known to have power electronics significance and can be directly computed from the other input features. To improve the models with these features a function transformer must be used which preprocesses the input features and adds the selected engineered features to the input vector. The estimators are then trained on the new, larger input vector and the estimator is configured so that when it is making predictions it also transforms the unknown input vector into a new vector with the engineered features.

The real input vector contains the input voltage, $V_{\text {in }}$, the output voltage, $V_{\text {out }}$, and the output current, $I_{\text {out }}$; however, there are many other properties that can be computed from this data that are known to have meaning in power electronics. Here six engineered features are considered as shown in Table 10.2.

Fig. 10.6 shows the correlation between the input features and the output variables. From 


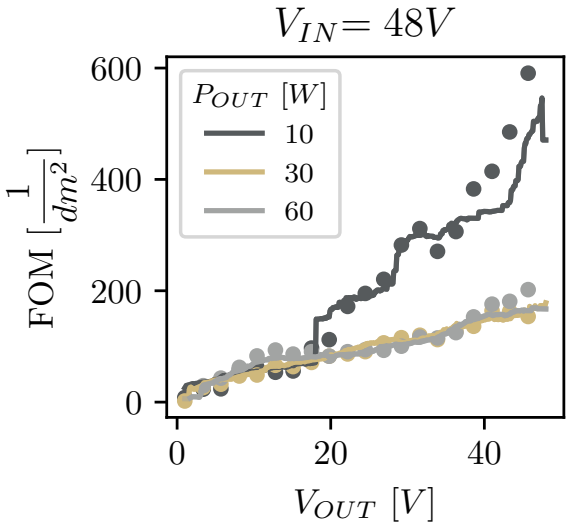

(a)

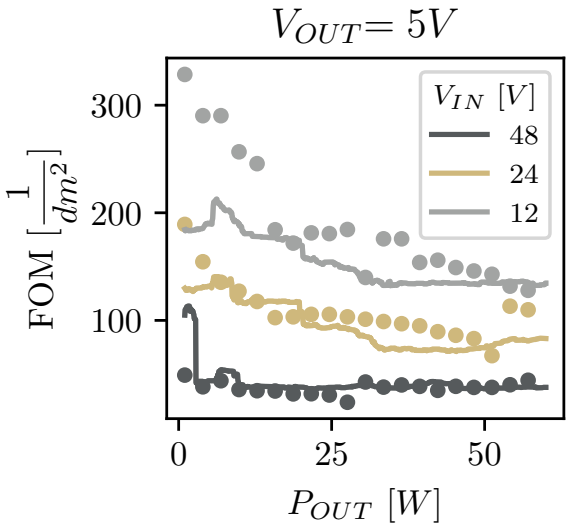

(d)

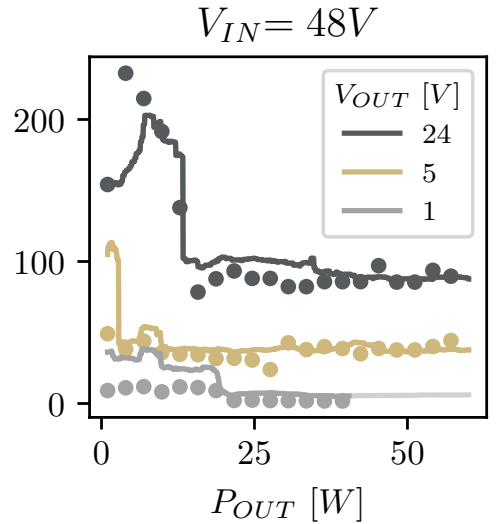

(b)

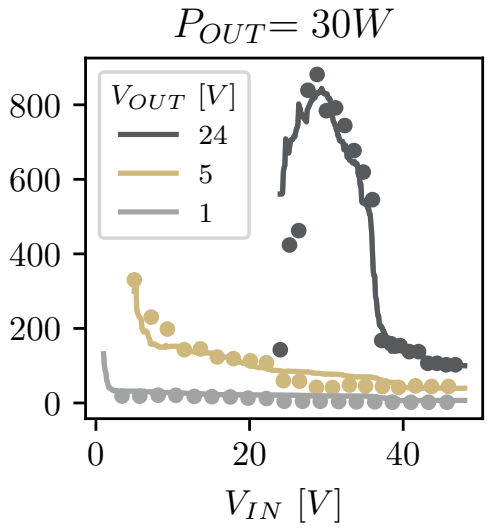

(e)

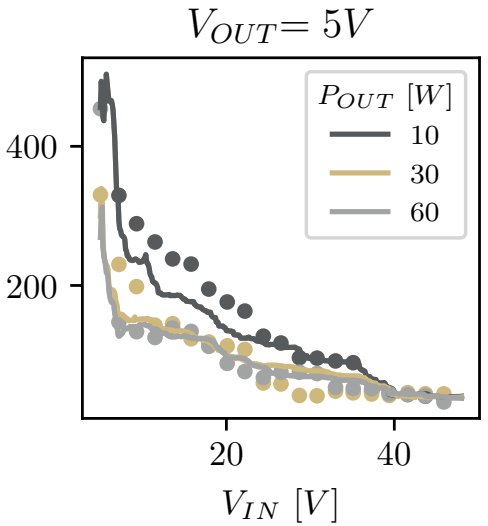

(c)

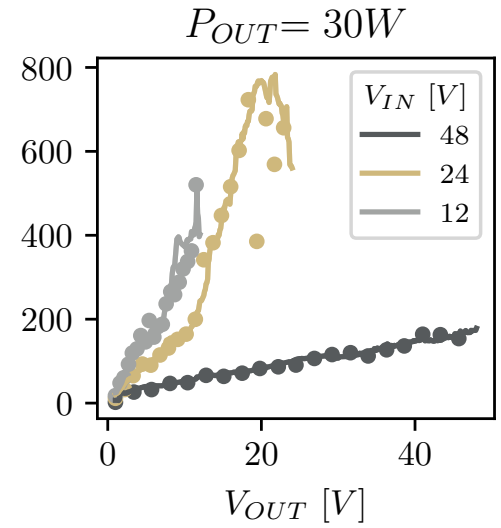

(f)

Figure 10.5: Performance of random forest estimator on typical converter sweeps compared to WEBENCH data. Solid line shows estimator predictions within feasible range and shaded lines show predictions beyond feasible range. Points are data directly from WEBENCH. Results show that the estimator is able to accurately follow the trends in the data points even when there are step changes in the converter FOM such as around $12 \mathrm{~W}$ for the $48-24 \mathrm{~V}$ converter. The classifier is also able to predict where in the sweep the results become infeasible such as around $30 \mathrm{~W}$ for the 48-1 V converter. 
Table 10.2: Considered Features

\begin{tabular}{lcc}
\hline Feature & Symbol & Expression \\
\hline \hline Power & $P$ & $V_{\text {out }} I_{\text {out }}$ \\
Conversion Ratio & $M$ & $\frac{V_{\text {in }}}{V_{\text {out }}}$ \\
Inverse Conversion Ratio & $M^{-1}$ & $\frac{V_{\text {out }}}{V_{\text {in }}}$ \\
Output Current Squared & $I_{\text {out }}^{2}$ & $\frac{I_{\text {out }}^{2}}{\text { Input Current }}$ \\
Output Conductance & $I_{\text {in }}$ & $\frac{V_{\text {out }} I_{\text {out }}}{V_{\text {in }}}$ \\
\hline
\end{tabular}

these results a few key relations are apparent. First, cost and area are very similar with a strong correlation between each variable and the $I_{\text {out }}, I_{\text {out }}^{2}$, and, $G_{\text {out }}$ features. Second, the FOM is most correlated with the inverse conversion ratio. Finally, feasibility has a strong negative correlation with $I_{\text {out }}, I_{\text {out }}^{2}, I_{\text {in }}$, and, $G_{\text {out }}$ which is not surprising as increases in each make the converter more difficult to realize.

To assess the performance of adding these features for regression, random forest and gradient boosting regressors are trained on every combination of considered engineered features using a converter dataset optimizing for FOM. The performance of each estimator is then compared using a five split cross validation. Notable results from this search of 64 possible new feature sets (including the empty set) are presented in Table 10.3. The feature set with the highest performing estimator contains the features $\left\{M, M^{-1}, I_{\text {out }}^{2}, I_{\text {in }}\right\}$; however, there are several feature sets that also perform comparably. The two with the fewest features are $\{P, M\}$ and $\left\{P, M^{-1}\right\}$ which have almost the same $r^{2}$ values. Each of those feature sets preforms slightly better than that not adding any new features; however, there is only a small separation and the means are within one standard deviation 


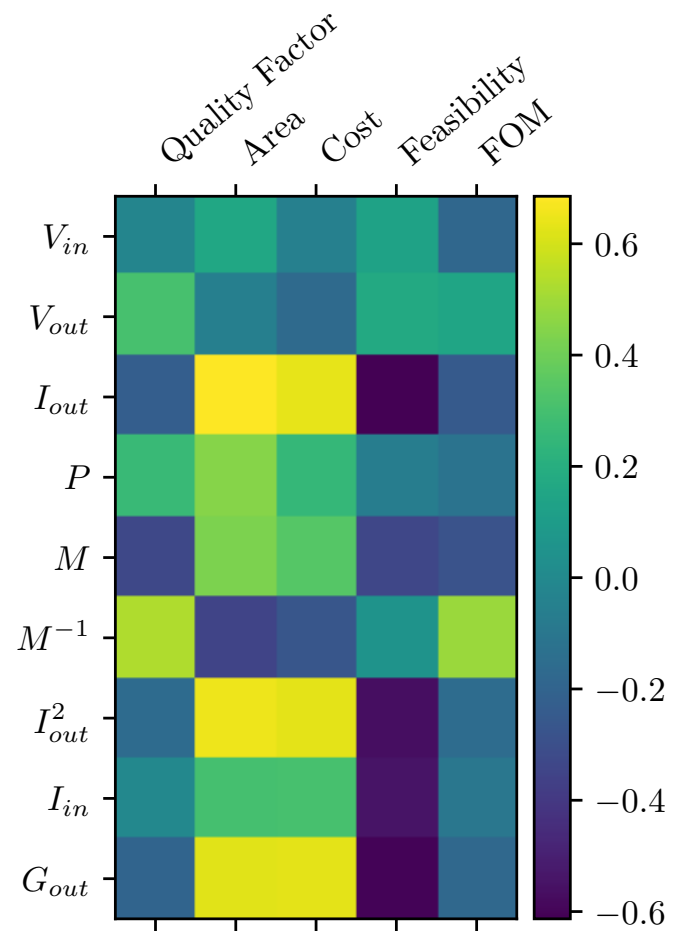

Figure 10.6: Correlation matrix for input and output variables including engineered features. The vertical axis is input features and the horizontal axis is output variables.

of one another.

The performance benefits for classification are also examined in the same way and the results are shown in Table 10.4.

\subsubsection{Extrapolation Performance}

Random forest and gradient boosting regressors are both inherently unable to extrapolate beyond their training data, the estimator can predict values outside of the maximum and minimum values of the training set. If the estimator is asked to predict the value of something that would have a value greater than its maximum training value it will only predict the maximum training value no matter how unrealistic that prediction is. In order to prevent this care should be taken to ensure that the estimator is not asked to estimate something that is outside of its training data. The most straightforward method of doing this is to classify converters outside the limits of the 
Table 10.3: Regression Feature Engineering Results

\begin{tabular}{ccc}
\hline New Feature Set & Mean $r^{2}$ & $r^{2}$ Deviation \\
\hline \hline$\left\{M, M^{-1}, I_{\text {out }}^{2}, I_{\text {in }}\right\}$ & 0.857 & 0.053 \\
\{\} & 0.828 & 0.031 \\
$\{P, M\}$ & 0.853 & 0.048 \\
$\left\{P, M^{-1}\right\}$ & 0.851 & 0.050 \\
$\left\{M^{\prime}\right\}$ & 0.845 & 0.055 \\
$\left\{M^{-1}\right\}$ & 0.842 & 0.052 \\
$\left\{I_{\text {in }}\right\}$ & 0.835 & 0.041 \\
$\left\{G_{\text {out }}\right\}$ & 0.824 & 0.040 \\
$\{P\}$ & 0.818 & 0.040 \\
$\left\{I_{\text {out }}^{2}\right\}$ & 0.816 & 0.031 \\
\hline
\end{tabular}

Table 10.4: Classification Feature Engineering Results

\begin{tabular}{cll}
\hline New Feature Set & Mean Specificity & Specificity Deviation \\
\hline \hline$\left\{M^{-1}, I_{\text {in }}\right\}$ & 0.900 & 0.122 \\
\{\} & 0.825 & 0.1 \\
$\{M\}$ & 0.875 & 0.111 \\
$\left\{M^{-1}\right\}$ & 0.875 & 0.111 \\
$\left\{I_{\text {in }}\right\}$ & 0.85 & 0.094 \\
$\left\{G_{\text {out }}\right\}$ & 0.85 & 0.094 \\
$\{P\}$ & 0.825 & 0.100 \\
$\left\{I_{\text {out }}^{2}\right\}$ & 0.825 & 0.100 \\
\hline
\end{tabular}


input vector as infeasible.

This is done in Fig. 10.7 where $P$ and $I_{\text {out }}$ are included in the classifier's input vector. Where the solid lines become transparent is where the classifier is predicting infeasibility. This technique does ensure that the estimator does not predict beyond its feasibility, but for these cases it is somewhat conservative. For the converters that reach infeasibility while the predicted area is still low they continue to accurately predict the area outside the feasible region to a certain extent. More sophisticated means of limiting extrapolation may be able to extend the prediction region further.

\section{$10.4 \quad$ Application Case Studies}

\subsubsection{Intermediate Bus Node Placement}

One application of dc-dc performance estimators is in the placement of IBNs. These intermediate conversion steps can improve the efficiency and reduce the size of high-ratio converters. However, determining the optimal number and placement of these stages can be computationally expensive. An example of the trends seen in these problems is shown in Fig. 10.8. These results are for a 48-1 V converter supplying a $30 \mathrm{~A}$ load and are generated using a random forest estimator. The results imply that losses are minimized when the IBN is between 15 and $20 \mathrm{~V}$ while area is minimized for IBNs between 3.5 and $6 \mathrm{~V}$. These results also show that the estimator is highly accurate at predicting the losses as the intermediate bus node changes, but that it somewhat underestimates the area as the placement changes. Nevertheless the estimator still accurately predicts the optimal placement for the system with the best FOM as shown in Fig. 10.9.

\subsubsection{Distribution Architecture Design}

Another application of estimators is in the design of complex dc distribution systems with multiple dc outputs as discussed throughout this thesis. When designing these systems there are a large number of possible converters and it is computationally intensive to evaluate them all using slow database sources such as WEBENCH. Estimators can narrow down the list of systems that 


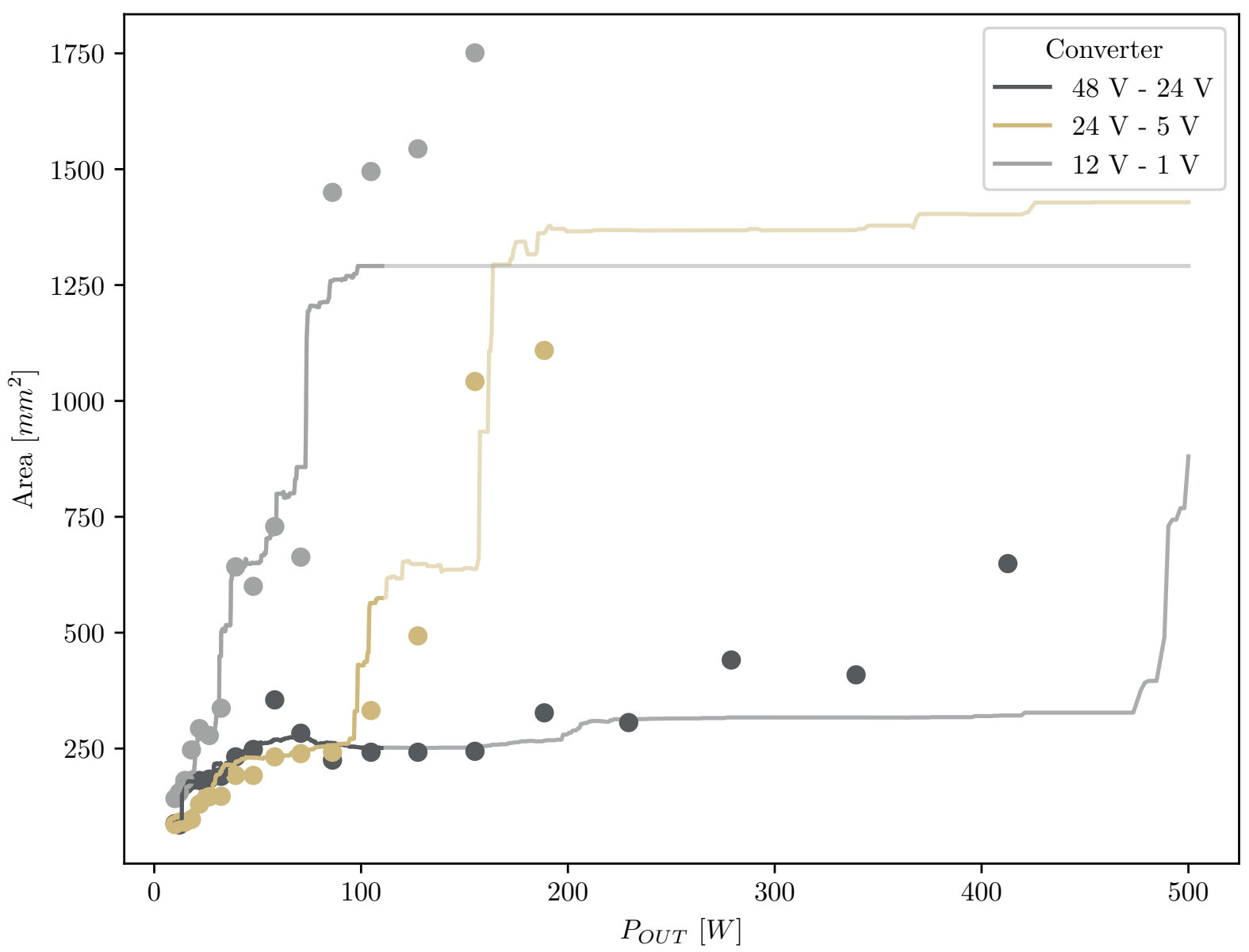

Figure 10.7: Performance of the estimator outside of its training data. The solid lines show area predictions from a random forest estimator trained on 800 converters as the output power increases. Where the lines become transparent is where the classifier prevents extrapolation by marking all inputs outside the training data as infeasible. While this does prevent extrapolation it is a conservative means of doing so as the regressor is able to accurately make predictions outside this feasibility region in certain cases. 


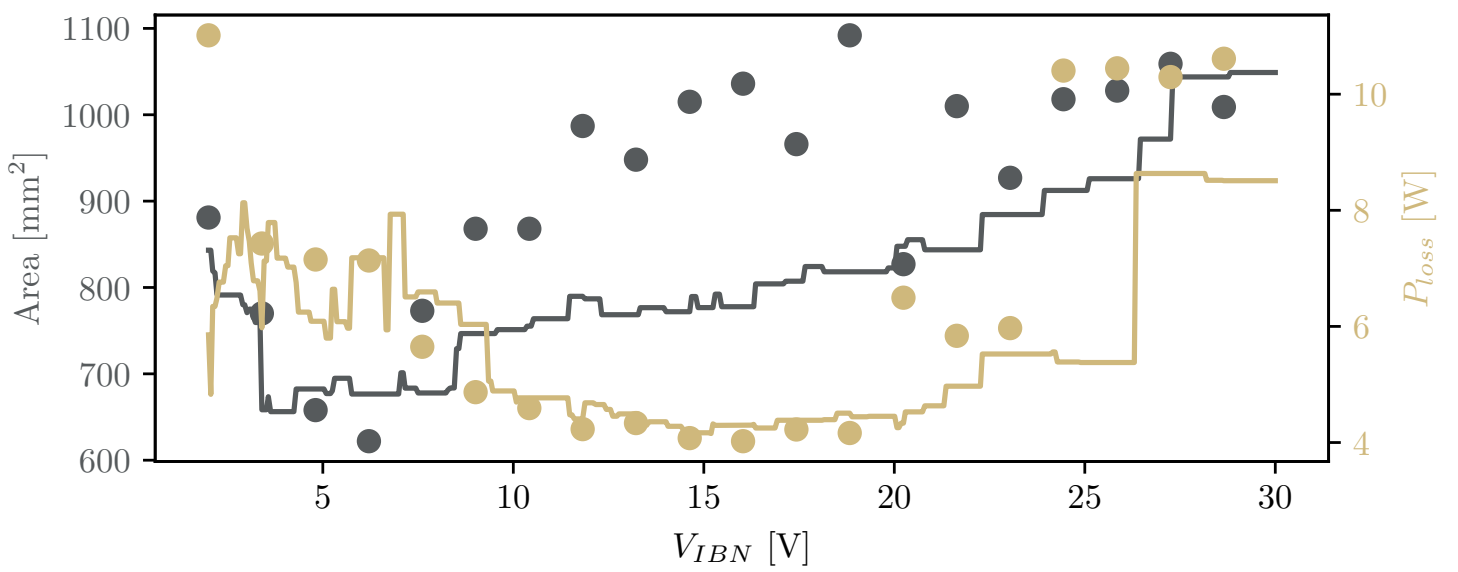

Figure 10.8: Area and losses for system with two converters as a function of intermediate bus node placement. System Input is $48 \mathrm{~V}$ and the output is $1 \mathrm{~V}$ at $30 \mathrm{~A}$. Generated using Gradient Boosting estimator trained on 800 commercially available converters. Solid lines show the estimator predictions and the points show the results for systems realized with WEBENCH [29] data.

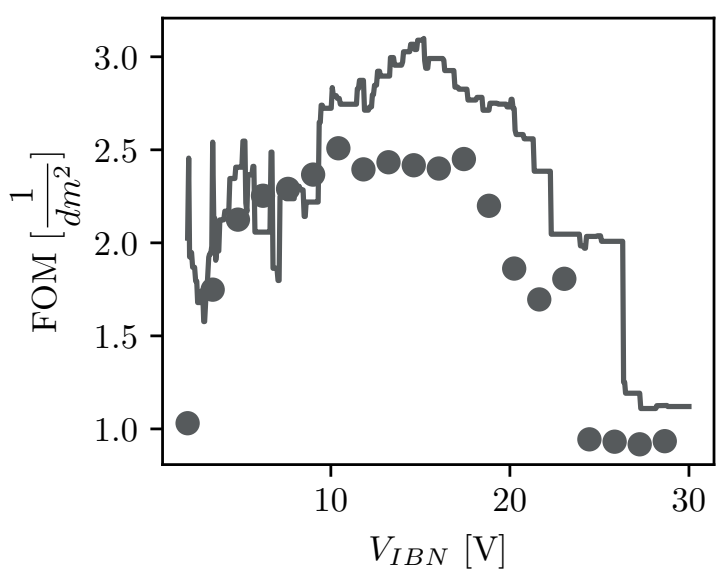

Figure 10.9: FOM for a system with two converters as a function of intermediate bus node placement. The line shows FOM predictions from a Gradient Boosting regressor and the points represent FOM for the system realized with converters from WEBENCH [29]. 


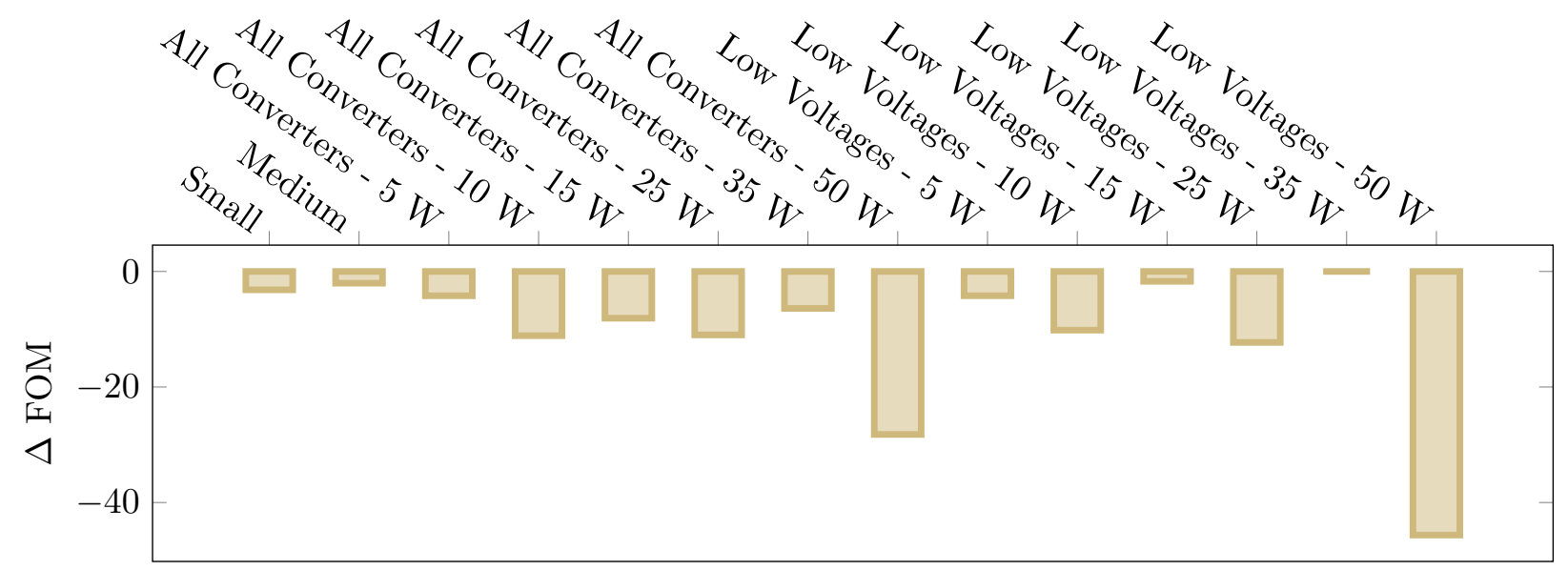

Figure 10.10: Performance comparison between estimator system designs and WEBENCH system designs. Each bar shows the decrease in performance for each test case when using the arrangement found with estimator data. All bars show a decrease as in the best case scenario the estimator finds the true optimal and has no decrease in performance.

must be evaluated with high quality data sources. As an example 14 dc distribution system design test cases are considered. For each test case the system is solved using converter performance data coming from both a random forest estimator and WEBENCH to determine characteristics for each converter in the distribution architecture. Once the best estimator constructed arrangement is determined, it is reevaluated with WEBENCH converters and compared with the true optimal as found by using the WEBENCH data source. Results for each of these test cases can be seen in Fig. 10.10 .

These results show that when the output power levels are low the error is less than $15 \%$. However when the individual output power levels are increased to $50 \mathrm{~W}$ the optimization performance decreases considerably. This can be explained by considering the training data used to generate the estimator. All training data is at most $100 \mathrm{~W}$; however, when multiple outputs in the system are $50 \mathrm{~W}$, converters in the system may need to process more than $100 \mathrm{~W}$ as they must supply power for their own load and their children's loads. Random forest estimators are unable to extrapolate beyond training data, so the maximum predicted area cannot exceed the largest training converter. When the estimator fueled optimization routine evaluates architectures that contain these high power converters, it will underestimate the area and therefore overestimate the FOM 
providing sub-optimal results. This can be seen clearly in Fig. 10.7 where the $48-24 \mathrm{~V}$ predictions rail out at around $1300 \mathrm{~mm}^{2}$ (The maximum training area is $1638 \mathrm{~mm}^{2}$ ). This indicates that care should be taken when using random forest estimators to ensure that they are not used with inputs well outside of the training data.

\subsection{Conclusions}

A variety of power electronics problems require estimation of the performance and properties of converters as part of their design procedure and Artificial Intelligence (AI) estimators can be used as part of this process. This chapter characterizes a sample of machine learning techniques applied to estimation of commercial dc-dc converters both to classify feasibility and to make real valued estimates of converter characteristics. Results show that machine learning estimators can accurately estimate the performance of commercially available converters and follow the trends as input voltage, output voltage, and output current are varied. Additionally, application examples show that results match well with high fidelity models when extrapolation beyond the range of training data is not required. 


\section{Chapter 11}

\section{Conclusions}

Complex electronic systems frequently require complex power distribution networks to supply power to their various subsystems. While prior work has extensively considered design and optimization of individual converter modules, very little research has examined the design and optimization of these dc-dc distribution architectures. This thesis presents a systematic approach to the design of dc-dc distribution architectures along with case studies and experimental results that showcase its performance. These results show significant increases in system performance using the presented techniques compared to the prior state of the art.

Additional techniques are presented for the optimization of very large systems. These systems are intractable using the permutation graph method, which is an exhaustive-search method, but can be solved with good performance using two other efficient methods that do not attempt to examine all possible system architectures. These techniques are described in detail and results are presented to show solution-time performance benefits in systems that can be solved using exhaustive search and improved solution performance when solving large systems as compared to a heuristic approach.

Finally, techniques for data-driven estimation of power electronic converter performance are

examined, their performance is characterized, and application examples are provided to show how this approach can be used in power electronics design. 


\subsection{Contributions}

(1) The permutation-graph method [10], [41] for design and optimization of dc-dc distribution architectures as outlined in Chapters 2 to 5 . The technique produces optimal systems through an exhaustive search of the design space to produce systems with improved performance over the state-of-the-art. Case studies (Chapter 8) and experimental results (Chapter 9) showcase the performance.

(2) Descent based optimization [71] as detailed in Chapter 6 defines a new design space structure and presents two techniques for moving through it with significant improvements in the number of arrangements that must be evaluated. Detailed analysis describes the design space in detail and provides theoretical performance estimations.

(3) A technique for moving through the design space using simulated annealing [49] is presented in Chapter 7. Power electronics specific details are presented and their performance in case studies is reported.

(4) Machine learning techniques for power electronics performance modeling [72] are examined and characterized in Chapter 10. Application examples showcase the technique for power electronics design.

\subsection{Future Work}

(1) Further improvements to descent and simulated annealing may be possible. Heuristically, weighting the design space may lead to more efficient movement as the techniques can influence the stochastic processes to some extent.

(2) Automated selection of the various optimization procedures with a heuristic may be beneficial. This will eliminate the requirement that the designer understand the difference between the various methods when optimizing the system. 
(3) Improvements to placement of IBNs are possible. Current placement strategies are static and do not allow the movement of IBNs once they are created.

(4) Significant work remains on converter performance models.

(a) Automatic generation of estimators for specific applications. Currently estimators are designed with designer intuition. Heuristics and automated procedures may allow this process to happen with no designer intervention.

(b) Application of performance models to novel converter topologies, not included among state-of-the-art commercially available converters

(c) Application of performance models to individual components could lead to improved design automation techniques at the converter model level

(d) Improved techniques for limiting extrapolation are needed to allow estimators to predict outside of their training data where accuracy is still good.

(e) Fusion of converter performance models with high fidelity models for system design. Current work examines them only separately, but there are likely performance benefits if the two techniques are combined. The speed of performance models in the early stages followed by the accuracy of high fidelity models towards the end of the design process. 


\section{Bibliography}

[1] L. M. Tolbert, "Power Electronics for Distributed Energy Systems and Transmission and Distribution Applications: Assessing the Technical Needs for Utility Applications," English, Oak Ridge National Lab. (ORNL), Oak Ridge, TN (United States), Tech. Rep. ORNL/TM2005/230, Dec. 2005.

[2] R.-J. Wai and K.-H. Jheng, "High-Efficiency Single-Input Multiple-Output DC-DC Converter," IEEE Transactions on Power Electronics, vol. 28, no. 2, pp. 886-898, Feb. 2013.

[3] J. D. Dasika, B. Bahrani, M. Saeedifard, A. Karimi, and A. Rufer, "Multivariable Control of Single-Inductor Dual-Output Buck Converters," IEEE Transactions on Power Electronics, vol. 29, no. 4, pp. 2061-2070, Apr. 2014.

[4] O. Ray, A. P. Josyula, S. Mishra, and A. Joshi, "Integrated Dual-Output Converter," IEEE Transactions on Industrial Electronics, vol. 62, no. 1, pp. 371-382, Jan. 2015.

[5] W. Huang, J. A. A. Qahouq, and Z. Dang, "CCM-DCM Power-Multiplexed Control Scheme for Single-Inductor Multiple-Output DC-DC Power Converter With No Cross Regulation," IEEE Transactions on Industry Applications, vol. 53, no. 2, pp. 1219-1231, Mar. 2017.

[6] T. Y. Goh and W. T. Ng, "Single Discharge Control for Single-Inductor Multiple-Output DC-DC Buck Converters," IEEE Transactions on Power Electronics, vol. 33, no. 3, pp. 2307-2316, Mar. 2018. 
[7] C. J. Solis and G. A. Rincón-Mora, "87\%-Efficient 330-mW 0.6-Mm Single-Inductor TripleOutput Buck-Boost Power Supply," IEEE Transactions on Power Electronics, vol. 33, no. 8, pp. $6837-6844$, Aug. 2018.

[8] Z. Dong, C. K. Tse, and S. Y. R. Hui, "Current-Source-Mode Single-Inductor MultipleOutput LED Driver With Single Closed-Loop Control Achieving Independent Dimming Function," IEEE Journal of Emerging and Selected Topics in Power Electronics, vol. 6 , no. 3, pp. 1198-1209, Sep. 2018.

[9] G. Chen, Y. Liu, X. Qing, M. Ma, and Z. Lin, "Principle and Topology Derivation of Single-Inductor Multi-Input Multi-Output DC-DC Converters," IEEE Transactions on Industrial Electronics, pp. 1-1, 2020.

[10] K. J. Goodrick and D. Maksimović, "Systematic Optimization of Multiple Voltage Domain DC Distribution Architectures," in 2018 IEEE 19th Workshop on Control and Modeling for Power Electronics (COMPEL), Jun. 2018.

[11] M. D. Seeman and S. R. Sanders, "Analysis and Optimization of Switched-Capacitor DC-DC Converters," IEEE Transactions on Power Electronics, vol. 23, no. 2, pp. 841-851, Mar. 2008.

[12] R. Ridley, C. Zhou, and F. Lee, "Application of nonlinear design optimization for power converter components," IEEE Transactions on Power Electronics, vol. 5, no. 1, pp. 2940, Jan. 1990.

[13] R. M. Burkart and J. W. Kolar, "Comparative $\eta-\rho-\sigma$ Pareto Optimization of Si and SiC Multilevel Dual-Active-Bridge Topologies With Wide Input Voltage Range," IEEE Transactions on Power Electronics, vol. 32, no. 7, pp. 5258-5270, Jul. 2017.

[14] R. Yu, G. K. Y. Ho, B. M. H. Pong, B. W.-K. Ling, and J. Lam, "Computer-Aided Design and Optimization of High-Efficiency LLC Series Resonant Converter," IEEE Transactions on Power Electronics, vol. 27, no. 7, pp. 3243-3256, Jul. 2012. 
[15] T. Neugebauer and D. Perreault, "Computer-aided optimization of DC/DC converters for automotive applications," IEEE Transactions on Power Electronics, vol. 18, no. 3, pp. 775-783, May 2003.

[16] S. H. Kang, D. Maksimović, and I. Cohen, "Efficiency Optimization in Digitally Controlled Flyback DC-DC Converters Over Wide Ranges of Operating Conditions," IEEE Transactions on Power Electronics, vol. 27, no. 8, pp. 3734-3748, Aug. 2012.

[17] J. Zhang, H. S.-H. Chung, A. W.-L. Lo, and T. Huang, "Extended Ant Colony Optimization Algorithm for Power Electronic Circuit Design," IEEE Transactions on Power Electronics, vol. 24, no. 1, pp. 147-162, Jan. 2009.

[18] J. Zhang, H. Chung, Wai-Lun Lo, S. Hui, and A.-M. Wu, "Implementation of a decoupled optimization technique for design of switching regulators using genetic algorithms," IEEE Transactions on Power Electronics, vol. 16, no. 6, pp. 752-763, Nov. 2001.

[19] S. E. De León-Aldaco, H. Calleja, and J. Aguayo Alquicira, "Metaheuristic Optimization Methods Applied to Power Converters: A Review," IEEE Transactions on Power Electronics, vol. 30, no. 12, pp. 6791-6803, Dec. 2015.

[20] A. Stupar, T. McRae, N. Vukadinovic, A. Prodic, and J. A. Taylor, "Multi-Objective Optimization of Multi-Level DC-DC Converters using Geometric Programming," IEEE Transactions on Power Electronics, pp. 1-1, 2019.

[21] H. Helali, D. Bergogne, J. Ben Hadj Slama, H. Morel, P. Bevilacqua, B. Allard, and O. Brevet, "Power converter's optimisation and design. Discrete cost function with genetic based algorithms," in 2005 European Conference on Power Electronics and Applications, Sep. 2005, 7 pp.-P.7.

[22] H. Helali, D. Bergogne, H. Morel, and J. B. H. Slama, "Power converter design methodology: Uses of multiple objective techniques for optimization of a $(42 / 14 \mathrm{~V})$ buck converter," in 4th International Conference on Integrated Power Systems, Jun. 2006, pp. 1-5. 
[23] P. Lefranc, X. Jannot, and P. Dessante, "Virtual prototyping and pre-sizing methodology for buck DC-DC converters using genetic algorithms," en, IET Power Electronics, vol. 5, no. 1, pp. 41-52, Jan. 2012.

[24] R. White, "Emerging on-board power architectures," in Eighteenth Annual IEEE Applied Power Electronics Conference and Exposition, 2003. APEC '03., vol. 2, Feb. 2003, 799-804 vol.2.

[25] — - "Using on-board power systems," in INTELEC 2004. 26th Annual International Telecommunications Energy Conference, Sep. 2004, pp. 234-240.

[26] L. Laguna, R. Prieto, J. A. Oliver, J. A. Cobos, H. Visairo, and P. Kumar, "Power conversion modeling methodology based on building block models," in 2009 IEEE Energy Conversion Congress and Exposition, Sep. 2009, pp. 3404-3410.

[27] L. Laguna, R. Prieto, J. A. Oliver, J. A. Cobos, and H. Visairo-Cruz, "Fast architecture generation and evaluation techniques for the design of large power systems," in 2010 IEEE Energy Conversion Congress and Exposition, Sep. 2010, pp. 3464-3469.

[28] H. Zhang and T. Kozono, "LTpowerPlanner: A System-Level Power Architecture Design Tool," en, p. 8,

[29] Texas Instruments, Webench Design Center Brochure, 2012.

[30] D. Jungnickel, Graphs, Networks, and Algorithms, en, 3rd ed, ser. Algorithms and Computation in Mathematics v. 5. Berlin ; New York: Springer, 2008.

[31] Arthur Cayley, "A Theorem On Trees," The Quarterly Journal of Pure and Applied Mathematics, vol. 23, p. 376, 1889.

[32] W. T. Tutte, "The dissection of equilateral triangles into equilateral triangles," en, Mathematical Proceedings of the Cambridge Philosophical Society, vol. 44, no. 4, pp. 463482, Oct. 1948.

[33] M. Muldoon, The Matrix-Tree Theorem, Class Lecture: MATH 20902, Class Lecture, School of Mathematics, University of Manchester, Manchester, Jan. 2017. 
[34] D. Costinett, D. Maksimovic, and R. Zane, "Design and Control for High Efficiency in High Step-Down Dual Active Bridge Converters Operating at High Switching Frequency," IEEE Transactions on Power Electronics, vol. 28, no. 8, pp. 3931-3940, Aug. 2013.

[35] O. Kirshenboim and M. M. Peretz, "High-Efficiency Nonisolated Converter With Very High Step-Down Conversion Ratio," IEEE Transactions on Power Electronics, vol. 32, no. 5, pp. 3683-3690, May 2017.

[36] K. I. Hwu, W. Z. Jiang, and Y. T. Yau, "Ultrahigh Step-Down Converter," IEEE Transactions on Power Electronics, vol. 30, no. 6, pp. 3262-3274, Jun. 2015.

[37] S. Saggini, O. Zambetti, R. Rizzolatti, M. Picca, and P. Mattavelli, "An Isolated QuasiResonant Multiphase Single-Stage Topology for 48-V VRM Applications," IEEE Transactions on Power Electronics, vol. 33, no. 7, pp. 6224-6237, Jul. 2018.

[38] C. Fei, M. H. Ahmed, F. C. Lee, and Q. Li, "Two-Stage 48 V-12 V/6 V-1.8 V Voltage Regulator Module With Dynamic Bus Voltage Control for Light-Load Efficiency Improvement," IEEE Transactions on Power Electronics, vol. 32, no. 7, pp. 5628-5636, Jul. 2017.

[39] K. Sano and H. Fujita, "Performance of a High-Efficiency Switched-Capacitor-Based Resonant Converter With Phase-Shift Control," IEEE Transactions on Power Electronics, vol. 26, no. 2, pp. 344-354, Feb. 2011.

[40] J. Zhu, R. Scheuss, and D. Maksimovic, "General Properties and Synthesis of Transformerless Stacked Active Bridge Converters," in 2019 20th Workshop on Control and Modeling for Power Electronics (COMPEL), Jun. 2019, pp. 1-6.

[41] K. J. Goodrick, J. Zhu, and D. Maksimović, "Systematic Optimization of Multiple-Output DC Distribution Architectures," IEEE Journal of Emerging and Selected Topics in Power Electronics, pp. 1-1, 2020.

[42] NXP, “I.MX53 System Development User's Guide,” en, Jun. 2015.

[43] Xilinx, "Kintex UltraScale FPGAs Data Sheet: DC and AC Switching Characteristics," en, 2019. 
[44] Xilinx, "Kintex-7 FPGAs Data Sheet: DC and AC Switching Characteristics," en, 2019.

[45] J. Edmonds, "Optimum branchings," en, Journal of Research of the National Bureau of Standards Section B Mathematics and Mathematical Physics, vol. 71B, no. 4, p. 233 , Oct. 1967.

[46] R. E. Tarjan, "Finding optimum branchings," en, Networks, vol. 7, no. 1, pp. 25-35, 1977.

[47] H. N. Gabow, Z. Galil, T. Spencer, and R. E. Tarjan, "Efficient algorithms for finding minimum spanning trees in undirected and directed graphs," en, Combinatorica, vol. 6, no. 2, pp. 109-122, Jun. 1986.

[48] J. A. Rice, Mathematical Statistics and Data Analysis, en, 3rd ed, ser. Duxbury Advanced Series. Belmont, CA: Thomson/Brooks/Cole, 2007.

[49] K. J. Goodrick, E. Dall'Anese, A. Butler, T. Byrd, and D. Maksimović, "Systematic Design and Optimization of Large DC Distribution Architectures Using Simulated Annealing," in 2021 IEEE Applied Power Electronics Conference and Exposition (APEC), Phoenix, AZ, USA, Jun. 21.

[50] S. Kirkpatrick, C. D. Gelatt, and M. P. Vecchi, "Optimization by Simulated Annealing," en, Science, vol. 220, no. 4598, pp. 671-680, May 1983.

[51] P. J. M. van Laarhoven and E. H. L. Aarts, Simulated Annealing: Theory and Applications, en. Dordrecht: Springer Netherlands, 1987.

[52] K. A. Dowsland and J. M. Thompson, "Simulated Annealing," en, in Handbook of Natural Computing, G. Rozenberg, T. Bäck, and J. N. Kok, Eds., Berlin, Heidelberg: Springer, 2012, pp. 1623-1655.

[53] V. Černý, "Thermodynamical approach to the traveling salesman problem: An efficient simulation algorithm," en, Journal of Optimization Theory and Applications, vol. 45, no. 1, pp. 41-51, Jan. 1985.

[54] B. Hajek, "Cooling Schedules for Optimal Annealing," Mathematics of Operations Research, vol. 13, no. 2, pp. 311-329, 1988. 
[55] E. Mese and D. A. Torrey, "An approach for sensorless position estimation for switched reluctance motors using artifical neural networks," IEEE Transactions on Power Electronics, vol. 17 , no. 1 , pp. 66-75, Jan. 2002.

[56] F. Kamran, R. G. Harley, B. Burton, T. G. Habetler, and M. A. Brooke, "A fast on-line neural-network training algorithm for a rectifier regulator," IEEE Transactions on Power Electronics, vol. 13, no. 2, pp. 366-371, Mar. 1998.

[57] Y. Zhao, R. Ball, J. Mosesian, J. de Palma, and B. Lehman, "Graph-Based Semi-supervised Learning for Fault Detection and Classification in Solar Photovoltaic Arrays," IEEE Transactions on Power Electronics, vol. 30, no. 5, pp. 2848-2858, May 2015.

[58] T. Wang, H. Xu, J. Han, E. Elbouchikhi, and M. E. H. Benbouzid, "Cascaded H-Bridge Multilevel Inverter System Fault Diagnosis Using a PCA and Multiclass Relevance Vector Machine Approach," IEEE Transactions on Power Electronics, vol. 30, no. 12, pp. 7006-7018, Dec. 2015.

[59] C. Wei, Z. Zhang, W. Qiao, and L. Qu, "An Adaptive Network-Based Reinforcement Learning Method for MPPT Control of PMSG Wind Energy Conversion Systems," IEEE Transactions on Power Electronics, vol. 31, no. 11, pp. 7837-7848, Nov. 2016.

[60] S. Zhou, L. Zhou, and P. Sun, "Monitoring Potential Defects in an IGBT Module Based on Dynamic Changes of the Gate Current," IEEE Transactions on Power Electronics, vol. 28, no. 3, pp. 1479-1487, Mar. 2013.

[61] S. Zhao and H. Wang, "Enabling Data-Driven Condition Monitoring of Power Electronic Systems With Artificial Intelligence: Concepts, Tools, and Developments," IEEE Power Electronics Magazine, vol. 8, no. 1, pp. 18-27, Mar. 2021.

[62] S. Zhao, F. Blaabjerg, and H. Wang, "An Overview of Artificial Intelligence Applications for Power Electronics," IEEE Transactions on Power Electronics, vol. 36, no. 4, pp. 46334658, Apr. 2021. 
[63] T. Guillod, P. Papamanolis, and J. W. Kolar, "Artificial Neural Network (ANN) Based Fast and Accurate Inductor Modeling and Design," IEEE Open Journal of Power Electronics, vol. 1, pp. 284-299, 2020.

[64] T. Guillod and J. W. Kolar, "From Brute Force Grid Search to Artificial Intelligence: Which Algorithms for Magnetics Optimization?: Workshop at Virtual PSMA Industry Session on Design of Magnetics for Different Circuit Topologies," en, in 35th IEEE Applied Power Electronics Conference (APEC 2020), Mar. 2020.

[65] J. H. Friedman, "Greedy function approximation: A gradient boosting machine.," en, Annals of Statistics, vol. 29, no. 5, pp. 1189-1232, Oct. 2001.

[66] Y. Freund and R. E. Schapire, "A Decision-Theoretic Generalization of On-Line Learning and an Application to Boosting," en, Journal of Computer and System Sciences, vol. 55, no. 1, pp. 119-139, Aug. 1997.

[67] L. Breiman, "Random Forests," en, Machine Learning, vol. 45, no. 1, pp. 5-32, Oct. 2001.

[68] C.-C. Chang and C.-J. Lin, "LIBSVM: A library for support vector machines," en, ACM Transactions on Intelligent Systems and Technology, vol. 2, no. 3, pp. 1-27, Apr. 2011.

[69] F. Pedregosa, G. Varoquaux, A. Gramfort, V. Michel, B. Thirion, O. Grisel, M. Blondel, P. Prettenhofer, R. Weiss, V. Dubourg, J. Vanderplas, A. Passos, D. Cournapeau, M. Brucher, M. Perrot, and É. Duchesnay, "Scikit-learn: Machine Learning in Python," Journal of Machine Learning Research, vol. 12, no. 85, pp. 2825-2830, 2011.

[70] G. E. Hinton, "Connectionist learning procedures," en, Artificial Intelligence, vol. 40, no. 1-3, pp. 185-234, Sep. 1989.

[71] K. J. Goodrick, A. Butler, T. Byrd, and D. Maksimović, "Efficient Design and Optimization of Large DC Distribution Architectures Using Descent Based Methods," in Design Methodologies for Power Electronics Conference, Bath, UK: IEEE, Jul. 21. 
[72] —_ "Machine Learning Estimators for Power Electronics Design and Optimization," in Design Methodologies for Power Electronics Conference, Bath, UK: IEEE, Jul. 21.

[73] K. J. Goodrick, G.-S. Seo, S. Mukherjee, J. Roy, R. Mallik, B. Majmunovic, S. Dutta, D. Maksimović, and B. Johnson, "LCOE Design Optimization Using Genetic Algorithm with Improved Component Models for Medium-Voltage Transformerless PV Inverters," in 2020 IEEE Energy Conversion Congress and Exposition (ECCE), Oct. 2020, pp. 22622267.

[74] G. Seo, S. Mukherjee, J. Roy, K. Goodrick, R. Mallik, B. Majmunovic, S. Dutta, D. Maksimovic, and B. Johnson, "Levelized-Cost-of-Electricity-Driven Design Optimization for MediumVoltage Transformerless Photovoltaic Converters," in Energy Conversion Congress and Exposition (ECCE), Baltimore, Md., United States: IEEE, Sep. 2019.

[75] A. Agrawal, S. Diamond, and S. Boyd, "Disciplined geometric programming," en, Optimization Letters, vol. 13, no. 5, pp. 961-976, Jul. 2019.

[76] A. Agrawal, R. Verschueren, S. Diamond, and S. Boyd, "A rewriting system for convex optimization problems," Journal of Control and Decision, vol. 5, no. 1, pp. 42-60, Jan. 2018.

[77] Analog Devices, DC/DC CONTROLLER Selection Guide, 2018.

[78] U. Badstuebner, J. Biela, D. Christen, and J. W. Kolar, "Optimization of a 5-kW Telecom Phase-Shift DC-DC Converter With Magnetically Integrated Current Doubler," IEEE Transactions on Industrial Electronics, vol. 58, no. 10, pp. 4736-4745, Oct. 2011.

[79] F. Balducci, D. Impedovo, and G. Pirlo, "Machine Learning Applications on Agricultural Datasets for Smart Farm Enhancement," en, Machines, vol. 6, no. 3, p. 38, Sep. 2018.

[80] D. Bertsimas and J. Tsitsiklis, "Simulated Annealing," Statistical Science, vol. 8, no. 1, pp. 10-15, Feb. 1993.

[81] S. P. Boyd and L. Vandenberghe, Convex Optimization, en. Cambridge, UK ; New York: Cambridge University Press, 2004. 
[82] S. Boyd, S.-J. Kim, L. Vandenberghe, and A. Hassibi, "A tutorial on geometric programming," en, Optimization and Engineering, vol. 8, no. 1, pp. 67-127, May 2007.

[83] S. Cabizza, L. Corradini, G. Spiazzi, and C. Garbossa, "Comparative Study of 48V-based Low-Power Automotive Architectures," in 2020 IEEE 21st Workshop on Control and Modeling for Power Electronics (COMPEL), Nov. 2020, pp. 1-8.

[84] H. Chen, H. Kim, R. Erickson, and D. Maksimović, "Electrified Automotive Powertrain Architecture Using Composite DC-DC Converters," IEEE Transactions on Power Electronics, vol. 32, no. 1, pp. 98-116, Jan. 2017.

[85] G. Chen, Y. Liu, X. Qing, and F. Wang, "Synthesis of Integrated Multiport DC-DC Converters With Reduced Switches," IEEE Transactions on Industrial Electronics, vol. 67, no. 6 , pp. 4536-4546, Jun. 2020.

[86] D. J. Costinett, "Analysis and design of high efficiency, high conversion ratio, DC-DC power converters," PhD Thesis, University of Colorado at Boulder, 2013.

[87] S. Diamond and S. Boyd, "CVXPY: A python-embedded modeling language for convex optimization," The Journal of Machine Learning Research, vol. 17, no. 83, pp. 1-5, Jan. 2016.

[88] R. W. A. A. D. Doncker, D. M. Divan, and M. H. Kheraluwala, "A three-phase soft-switched high-power-density DC/DC converter for high-power applications," IEEE Transactions on Industry Applications, vol. 27, no. 1, pp. 63-73, Jan. 1991.

[89] A. E. Eiben and J. E. Smith, Introduction to Evolutionary Computing, en, 2. ed, ser. Natural Computing Series. Heidelberg: Springer, 2015.

[90] R. W. Erickson and D. Maksimović, Fundamentals of Power Electronics, en. Cham: Springer International Publishing, 2020.

[91] H. N. Gabow, Finding All Spanning Trees of Undirected and Directed Graphs ; CU-CS-103-77, http://id.loc.gov/vocabulary/iso639-2/eng.

[92] J. Gao, Machine Learning Applications for Data Center Optimization. 2014. 
[93] T. Hastie, R. Tibshirani, and J. Friedman, The Elements of Statistical Learning, en, ser. Springer Series in Statistics. New York, NY: Springer New York, 2009.

[94] M. N. Kheraluwala, R. W. Gascoigne, D. M. Divan, and E. D. Baumann, "Performance characterization of a high-power dual active bridge DC-to-DC converter," IEEE Transactions on Industry Applications, vol. 28, no. 6, pp. 1294-1301, Nov. 1992.

[95] I. Kononenko, "Machine learning for medical diagnosis: History, state of the art and perspective," en, Artificial Intelligence in Medicine, vol. 23, no. 1, pp. 89-109, Aug. 2001.

[96] B. Korte and J. Vygen, "Spanning Trees and Arborescences," en, in Combinatorial Optimization: Theory and Algorithms, ser. Algorithms and Combinatorics, B. Korte and J. Vygen, Eds., Berlin, Heidelberg: Springer, 2012, pp. 131-155.

[97] Y. Kuai and P. L. Chapman, "Comprehensive and practical optimization of voltage regulator modules," in 2011 IEEE Power and Energy Conference at Illinois, Feb. 2011, pp. 1-6.

[98] S. Markkassery, A. Saradagi, A. D. Mahindrakar, L. N, and R. Pasumarthy, "Modelling, design and control of non-isolated single-input multi-output Zeta-Buck-Boost converter," IEEE Transactions on Industry Applications, pp. 1-1, 2020.

[99] Maxim Integrated, Mobile Power Product Selector Guide, Jul. 2019.

[100] D. Menzi, D. Bortis, G. Zulauf, M. Heller, and J. W. Kolar, "Novel iGSE-C Loss Modelling of X7R Ceramic Capacitors,” IEEE Transactions on Power Electronics, pp. 1-1, 2020.

[101] M. Mirjafari and R. S. Balog, "Multi-objective design optimization of renewable energy system inverters using a Descriptive language for the components," in 2011 Twenty-Sixth Annual IEEE Applied Power Electronics Conference and Exposition (APEC), Mar. 2011, pp. 1838-1845.

[102] M. R. Mojallizadeh and M. A. Badamchizadeh, "Hybrid Control of Single-Inductor MultipleOutput Converters," IEEE Transactions on Industrial Electronics, vol. 66, no. 1, pp. 451-458, Jan. 2019. 
[103] M. Muldoon, Representing Graphs, Class Lecture: MATH 20902, Class Lecture, School of Mathematics, University of Manchester, Manchester, Mar. 2019.

[104] M. M., "Chip Multilayer Ceramic Capacitors for General," en, 2020.

[105] N.-S. Pham, T. Yoo, T. T.-H. Kim, C.-G. Lee, and K.-H. Baek, "A 0.016 mV/mA CrossRegulation 5-Output SIMO DC-DC Buck Converter Using Output-Voltage-Aware Charge Control Scheme," IEEE Transactions on Power Electronics, vol. 33, no. 11, pp. 96199630, Nov. 2018.

[106] J.-C. Picard and M. Queyranne, "The Time-Dependent Traveling Salesman Problem and Its Application to the Tardiness Problem in One-Machine Scheduling," Operations Research, vol. 26 , no. 1 , pp. 86-110, 1978.

[107] M. B. F. Prieto, S. P. Litran, E. D. Aranda, and J. M. E. Gomez, "New Single-Input, Multiple-Output Converter Topologies: Combining Single-Switch Nonisolated dc-dc Converters for Single-Input, Multiple-Output Applications," IEEE Industrial Electronics Magazine, vol. 10, no. 2, pp. 6-20, Jun. 2016.

[108] K. Raggl, T. Nussbaumer, G. Doerig, J. Biela, and J. W. Kolar, "Comprehensive Design and Optimization of a High-Power-Density Single-Phase Boost PFC," IEEE Transactions on Industrial Electronics, vol. 56, no. 7, pp. 2574-2587, Jul. 2009.

[109] S. Rahman and F. C. Lee, "Nonlinear program based optimization of boost and buck-boost converter designs," in 1981 IEEE Power Electronics Specialists Conference, Jun. 1981, pp. 180-191.

[110] R. B. Ridley and F. C. Lee, "Practical nonlinear design optimization tool for power converter components," in 1987 IEEE Power Electronics Specialists Conference, Jun. 1987, pp. $314-323$.

[111] V. Roederstein, "General Technical Information," en, p. 8,

[112] G. Rozenberg, T. Bäck, and J. N. Kok, Eds., Handbook of Natural Computing, en. Berlin, Heidelberg: Springer Berlin Heidelberg, 2012. 
[113] C. Schaef, J. S. Rentmeister, and J. Stauth, "Multimode Operation of Resonant and Hybrid Switched-Capacitor Topologies," IEEE Transactions on Power Electronics, pp. 1-1, 2018 .

[114] F. M. Schaubauer and R Blumkin, "Thermal Resistance, Power Dissipation and Current Rating for Ceramic and Porcelain Multilayer Capacitors," en, p. 12,

[115] J Schoiswohl, "Linear Mode Operation and Safe Operating Diagram of Power-MOSFETs," en, p. 13,

[116] T. B. Soeiro, J. Mühlethaler, J. Linnér, P. Ranstad, and J. W. Kolar, "Automated Design of a High-Power High-Frequency LCC Resonant Converter for Electrostatic Precipitators," IEEE Transactions on Industrial Electronics, vol. 60, no. 11, pp. 4805-4819, Nov. 2013.

[117] A. L. Stein, P. A. Kyaw, J. Feldman-Stein, and C. R. Sullivan, "Scaling of parasitic capacitance with magnetic component physical size," in 2018 IEEE 19th Workshop on Control and Modeling for Power Electronics (COMPEL), Jun. 2018, pp. 1-8.

[118] TDK, "Film Capacitors - General technical information," en, p. 41, Jun. 2018.

[119] Texas Instruments, Power Management Guide, 2018.

[120] W. T. Tutte, Graph Theory, ser. Encyclopedia of Mathematics and Its Applications ; Section, Combinatorics v. 21. Reading, Mass: Addison-Wesley Pub, 1984.

[121] H. Visairo, M. A. Medina, and J. M. Ramirez, "Use of evolutionary algorithms for design optimization of power converters," in CONIELECOMP 2012, 22nd International Conference on Electrical Communications and Computers, Feb. 2012, pp. 268-272.

[122] R.-J. Wai and Z.-F. Zhang, "High-Efficiency Single-Input Triple-Outputs DC-DC Converter With Zero-Current Switching,” IEEE Access, vol. 7, pp. 84 952-84 966, 2019.

[123] H. Wang and M. Song, "Ckmeans.1d.dp: Optimal k-means Clustering in One Dimension by Dynamic Programming," The R Journal, vol. 3/2, pp. 29-33, Dec. 2011. 
[124] B. Wang, L. Xian, V. R. K. Kanamarlapudi, K. J. Tseng, A. Ukil, and H. B. Gooi, "A Digital Method of Power-Sharing and Cross-Regulation Suppression for Single-Inductor MultipleInput Multiple-Output DC-DC Converter," IEEE Transactions on Industrial Electronics, vol. 64, no. 4, pp. 2836-2847, Apr. 2017.

[125] Y. Xiong, S. Sun, H. Jia, P. Shea, and Z. John Shen, "New Physical Insights on Power MOSFET Switching Losses," IEEE Transactions on Power Electronics, vol. 24, no. 2, pp. 525-531, Feb. 2009. 


\section{Appendix A}

\section{Full Tree Generation Pseudocode}

While the pseudocode shown in Algorithm 5.1 is useful for describing the intuitive basis for the tree generation process, it excludes details necessary to fully implement the proposed procedure. In the complete version two lists of graphs must be returned, one for bucking trees and one for boosting trees. The algorithm must also take into account the placement of super and intermediate bus nodes. A complete pseudocode taking all of these factors into account is shown in Algorithm A.1. 


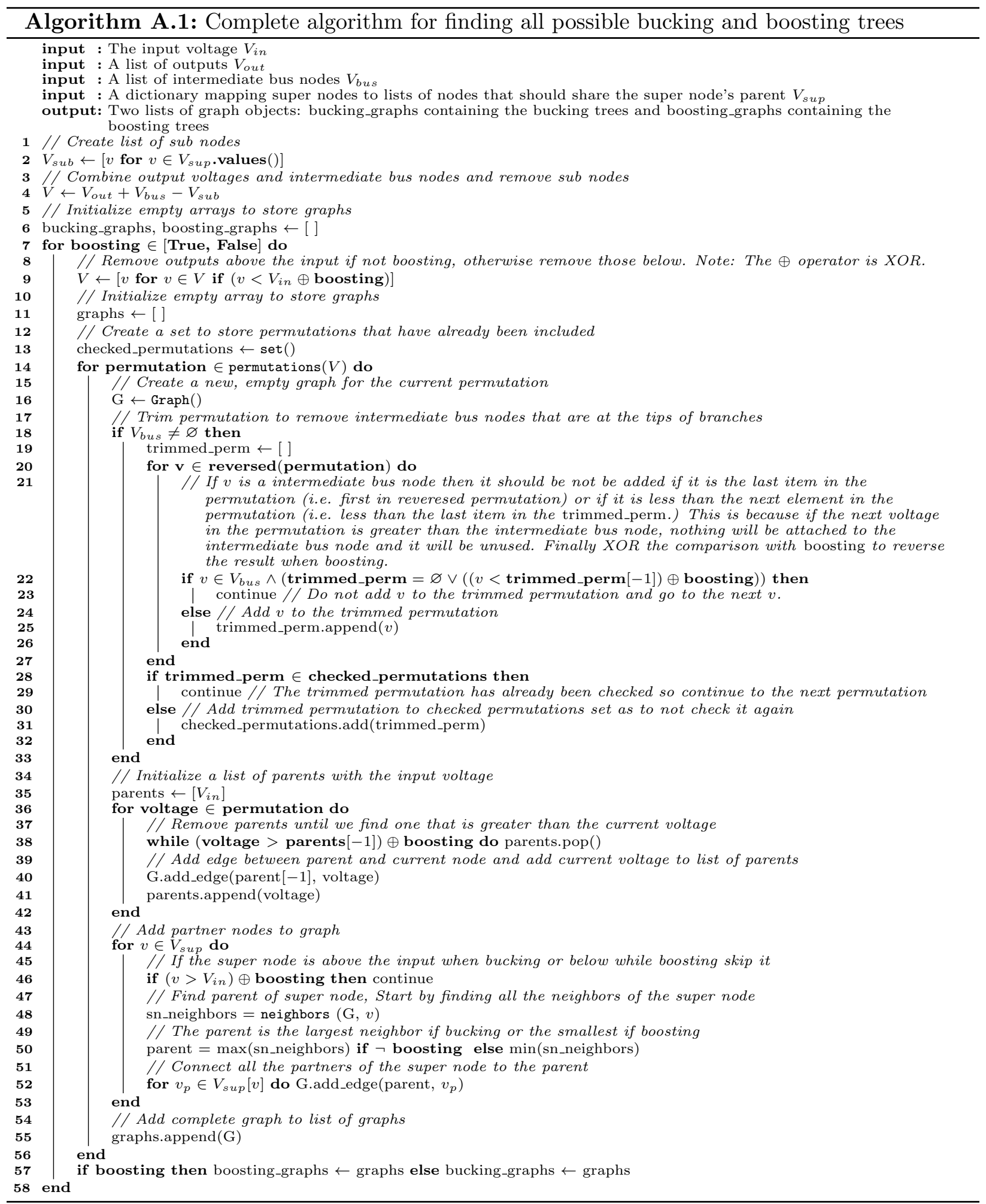

